\title{
Laser Spectroscopic Investigations of Praseodymium I Transitions: New Energy Levels
}

\author{
Zaheer Uddin, ${ }^{1,2,3}$ Driss El Bakkali, ${ }^{4}$ B. Gamper, ${ }^{1}$ Shamim Khan, ${ }^{1}$ Imran Siddiqui, ${ }^{1,3}$ \\ G. H. Guthöhrlein, ${ }^{4}$ and L. Windholz ${ }^{1}$ \\ ${ }^{1}$ Institute for Experimental Physics, University of Technology Graz, Petersgasse 16, 8010 Graz, Austria \\ ${ }^{2}$ Department of Physics, Yanbu University College, 51000 Yanbu Al Sanaiyah, Saudi Arabia \\ ${ }^{3}$ Department of Physics, University of Karachi, 75270 Karachi, Pakistan \\ ${ }^{4}$ Laboratorium für Experimentalphysik, Helmut-Schmidt-Universität, Universität der Bundeswehr Hamburg, Holstenhofweg 85, \\ 22043 Hamburg, Germany
}

Correspondence should be addressed to L. Windholz, windholz@tugraz.at

Received 12 April 2012; Accepted 13 June 2012

Academic Editor: Zoran Ikonic

Copyright () 2012 Zaheer Uddin et al. This is an open access article distributed under the Creative Commons Attribution License, which permits unrestricted use, distribution, and reproduction in any medium, provided the original work is properly cited.

We report the discovery of about 140 new energy levels of the neutral praseodymium atom, found by means of laser-induced fluorescence spectroscopy. Their energy has been determined with an uncertainty of $0.010 \mathrm{~cm}^{-1}$ using a wave number calibrated Fourier-transform spectrum.

\section{Introduction}

This work is a continuation of our systematic investigation of the praseodymium atomic spectrum (Pr I) with the goals of a more complete knowledge of its energy level scheme and to widen the classification of its spectral lines.

Praseodymium belongs to the rare earth elements, which have in common an open $4 \mathrm{f}$ electron shell. In nature one finds only one stable isotope, ${ }^{141} \mathrm{Pr}_{59}$, with the electronic ground state $[\mathrm{Xe}] 4 \mathrm{f}^{3} 6 \mathrm{~s}^{2},{ }^{4} \mathrm{I}_{9 / 2}^{\circ}$ and nuclear spin quantum number $\mathrm{I}=5 / 2$. Its nuclear magnetic dipole moment is $\mu_{\mathrm{I}}=4.2754(5) \mu_{\mathrm{N}}[1]$, and its nuclear electric quadrupole moment $\mathrm{Q}=-0.0024 \mathrm{~b}[2]$. Due to the open $4 \mathrm{f}$ shell, Pr has a huge number of energy levels. This in turn leads to a very rich line spectrum.

In 1978, the electronic levels discovered by several authors were collected and published, see [3]. Later, great progress was achieved by Ginibre [4-6]. She evaluated high resolution Fourier transform (FT) spectra, thereby discovering a large number of electronic levels of Pr I and Pr II and determined their parities, angular momenta, and hyperfine (hf) constants. Using an atomic-beam-magnetic resonance method, hf constants of many known low-lying metastable levels were determined with high accuracy by
Childs and Goodman [7]. Investigation of the hf structure of Pr I lines was performed later by Kuwamoto et al. [8], Krzykowski et al. [9], and Furmann et al. [10]. Our group has been concerned with investigations of the hf structure of Pr I lines since 1999. First values of hf constants have been published in [11]. Later on we concentrated mainly on the discovery of unknown energy levels. Some of the results are published in [12-15].

The spectrum and level structure of the Pr atom are of astrophysical interest $[16,17]$ and of course are indispensable for a thorough theoretical description of the level scheme [11].

\section{Experimental Details}

Our investigations are based on a combination of laserinduced fluorescence (LIF) and Fourier-transform (FT) spectroscopy. Spectral lines of Pr were excited by laser light, generated by means of a tunable single-frequency dye laser system. Our source of free atoms was a dc hollow cathode discharge, which produced free Pr atoms by cathode sputtering. Due to the collision processes within the $\mathrm{Ar}$ plasma, also high-lying Pr levels are populated, thus the laser 


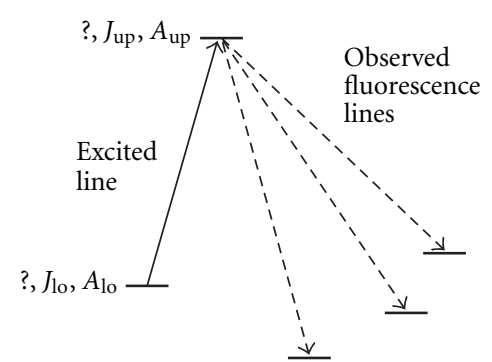

(a)

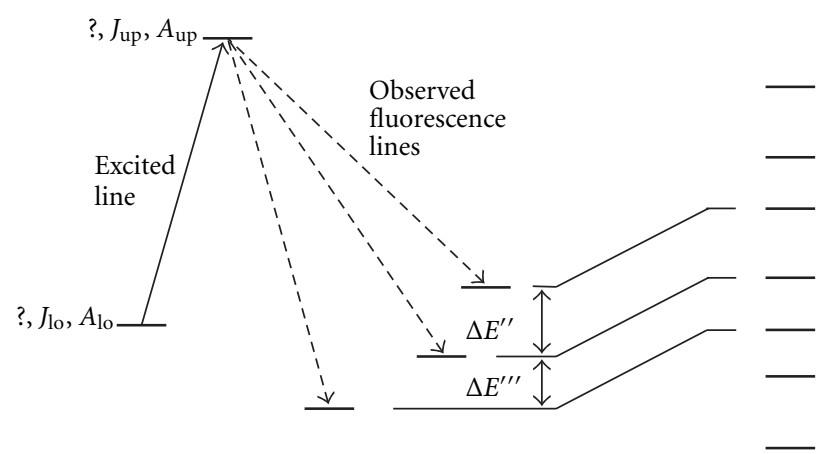

(c)

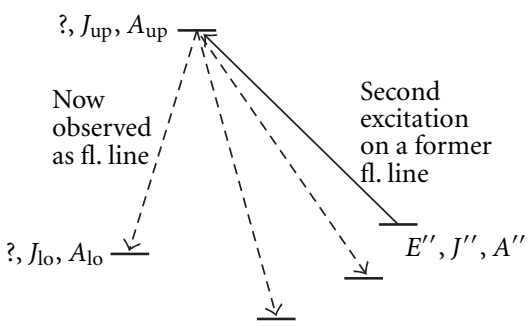

(b)

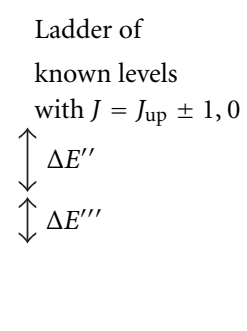

FIGURE 1: (a) Excitation of a transition for which both combining levels are unknown. (b) Identification of a known lower level by excitation of a former fluorescence line. (c) Finding of the lower levels of fluorescence lines by means of energy differences. See text for further details.

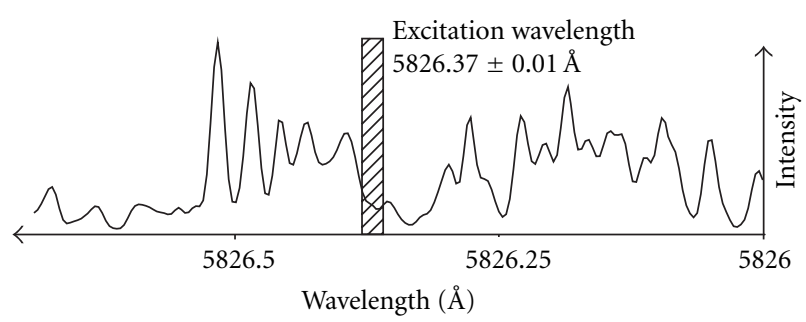

Figure 2: Part of the FT spectrum. Excitation cg wavelength $\lambda=$ $5826.37 \pm 0.01 \AA$. The excited hf pattern is not present in the FT spectrum. The cg wavelength of the line left of the marked range is $\lambda=5826.461 \AA$.

light could excite also atoms being in states with energies of up to $25000 \mathrm{~cm}^{-1}$. A detailed description of the experimental setup is given in [13].

First the laser wavelength was set to a selected value (extracted either from a wavelength table [18] or from a FT spectrum). The light emitted from the discharge was dispersed by a monochromator and detected by a photomultiplier. By scanning the monochromator wavelength, we could find laser-induced fluorescence signals with the help of a phase-sensitive Lock-In amplifier. This was possible because only laser-induced fluorescence light, emitted together with the complete Ar- and Pr-spectrum of the discharge, is modulated bearing the chopping frequency of the exciting laser beam.

Then we set the monochromator to one of the fluorescence lines and scanned the laser frequency over the hyperfine structure of the spectral line. In this way, we recorded the hf pattern of the excited transition.

\section{Data Evaluation}

Evaluating the recorded hf pattern, we determined the values of total angular momentum $J$ and hyperfine constants A of the levels involved in the investigated transition (in most cases, the B-factors could be neglected due to the small quadrupole moment of the Pr nucleus). This information, together with an estimated center-of-gravity (cg) wave number of the excited transition and the measured wavelengths of all fluorescence lines, usually allowed the determination of the energy of the excited level.

In most cases, the lower level involved in the transition could be identified on the basis of $J$ and $A$. Then a hypothetical upper level is introduced; its energy is calculated by adding the cg wave number to the energy of the lower level. Since the $J$-value of the new upper level is known from the evaluation of the observed hf structure, a list of possible transitions to known lower levels can be calculated. If this list explains the observed fluorescence lines, one can assume that a new upper level has been found.

Sometimes it turned out, that the fluorescence lines could be attributed to the decay of an already known upper level. In this case we had to assume, that the lower level of the transition is not yet known. The energy of the new lower level could be found by subtracting the cg wave number from the energy of this known upper level.

The situation was more complicated in cases where neither the lower nor the upper level of the excited transition 


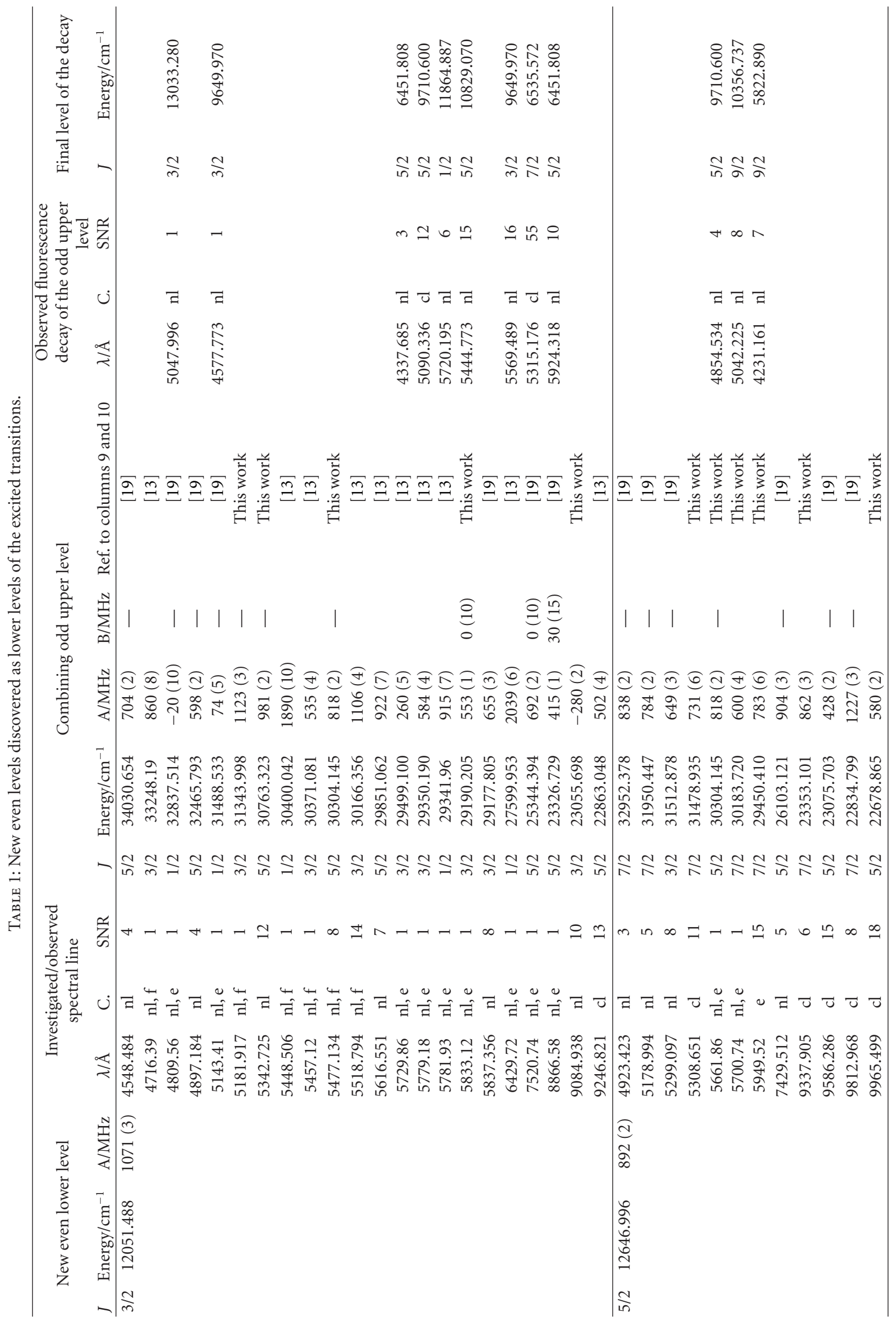




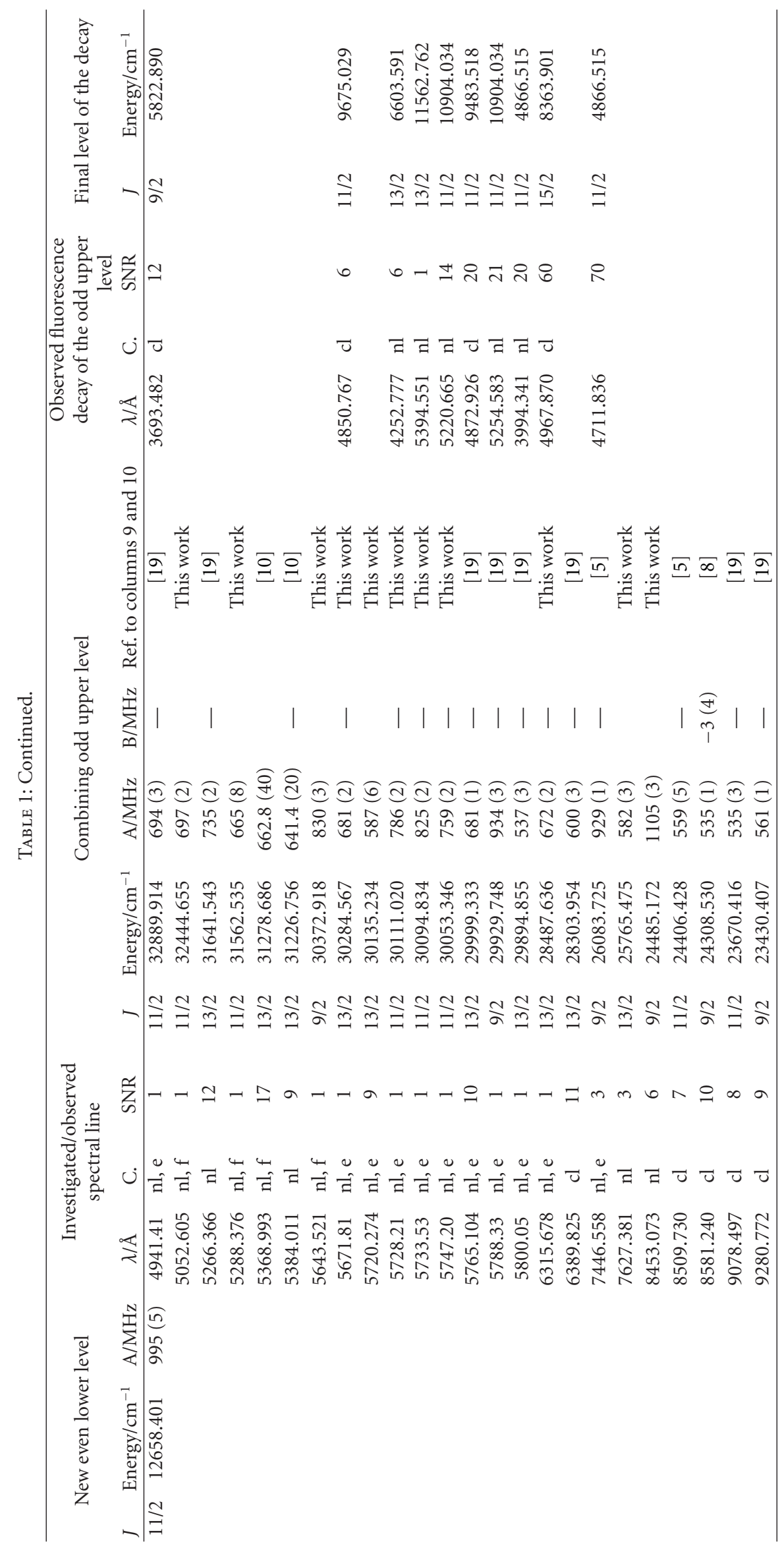




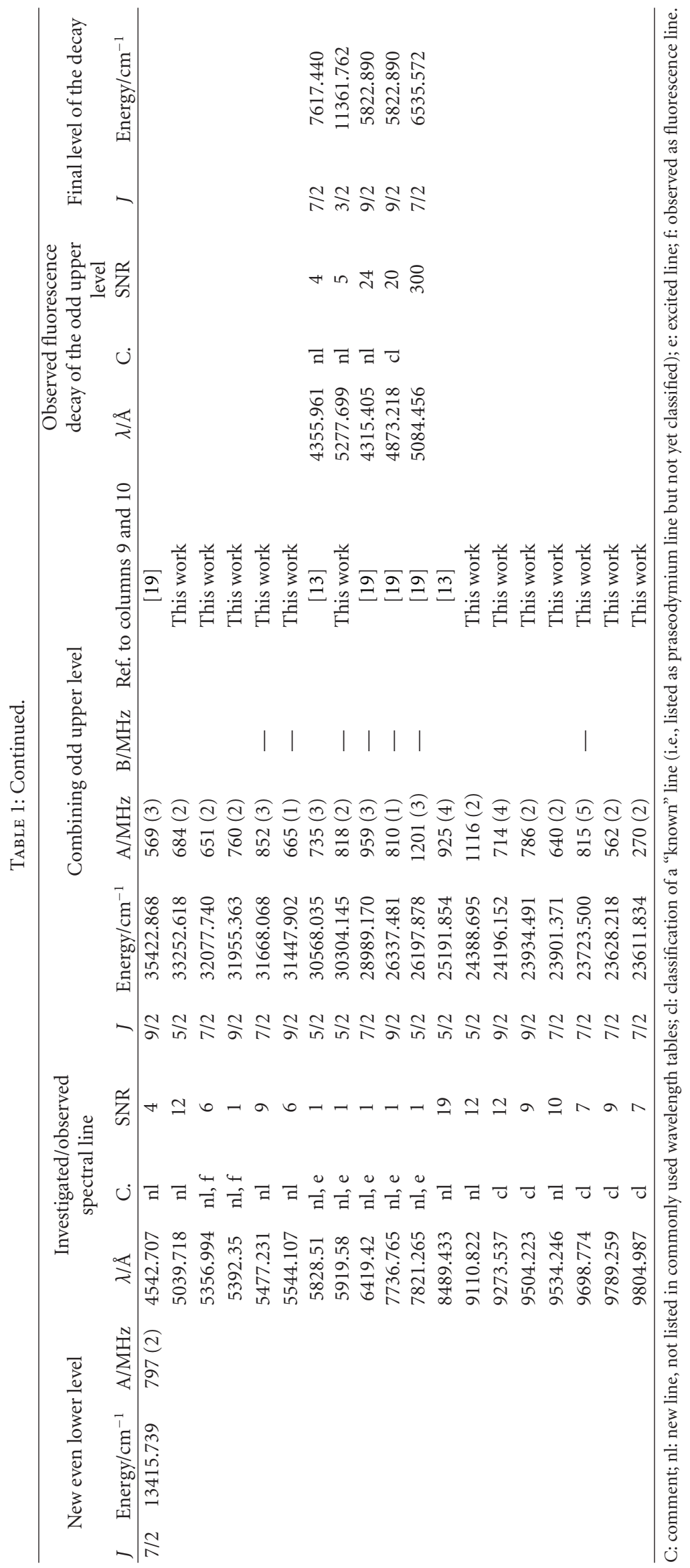




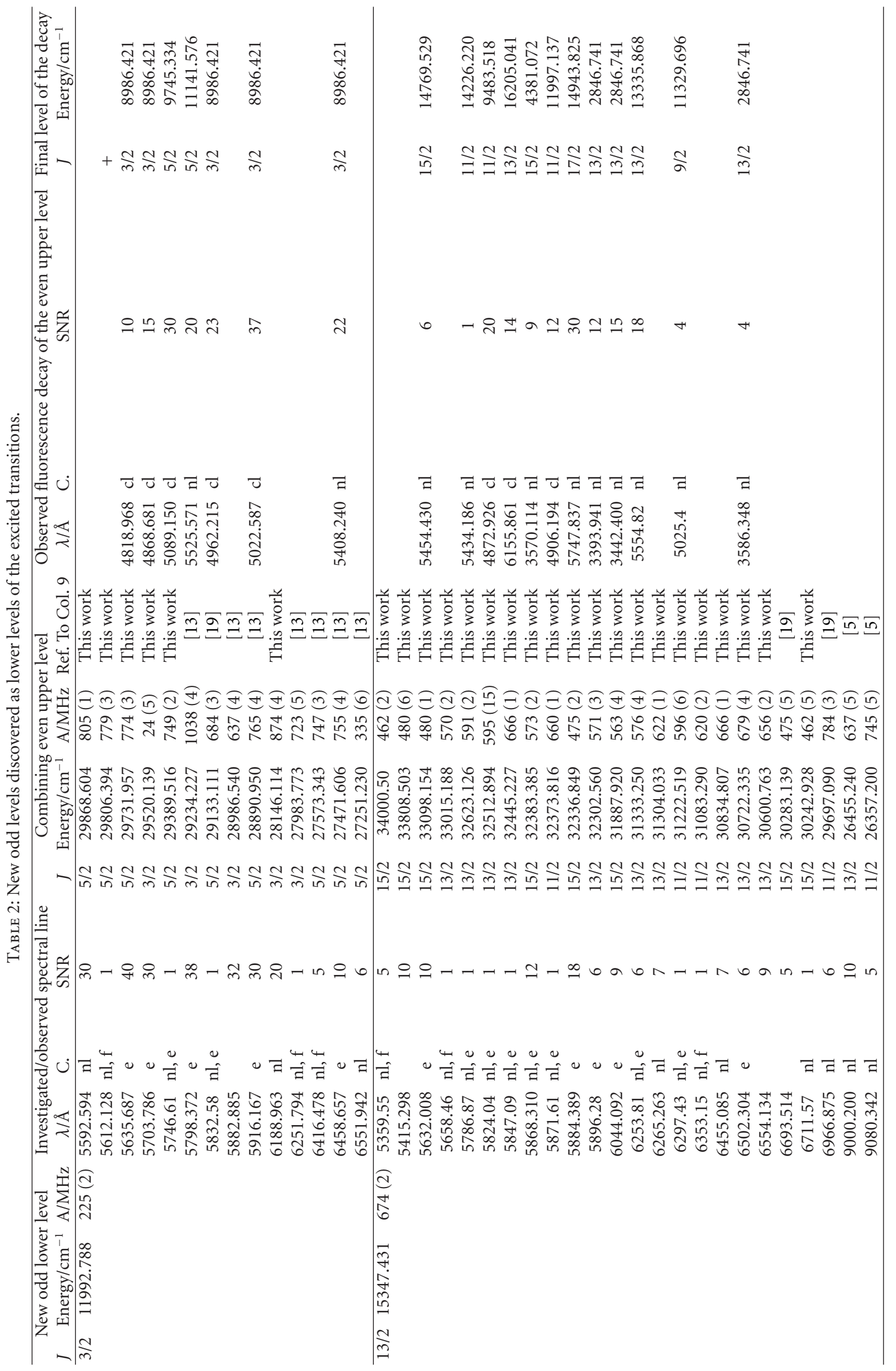




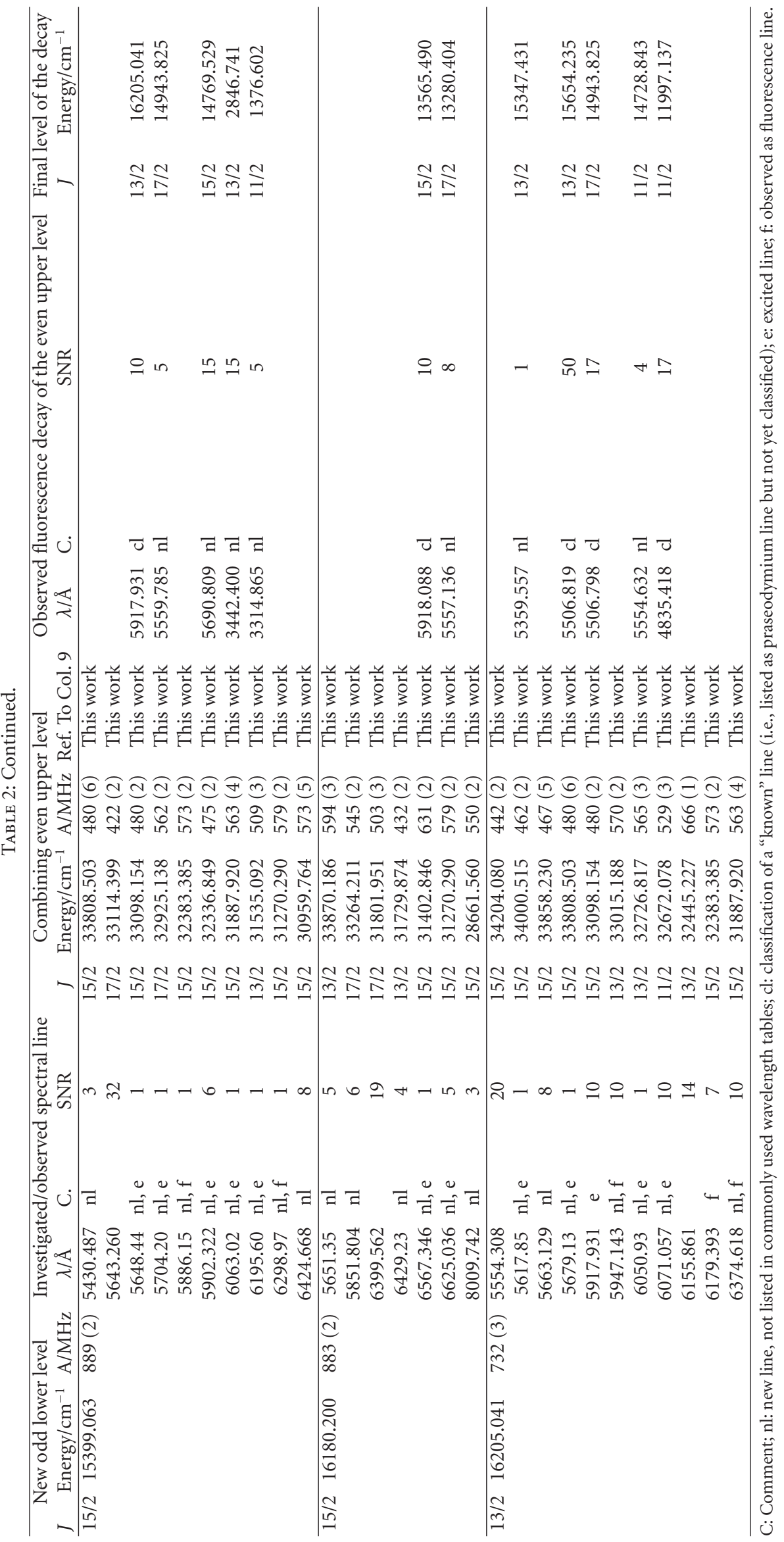


TABLE 3: New levels discovered as upper levels of the excited transitions.

\begin{tabular}{|c|c|c|c|c|c|}
\hline$J$ & Energy $/ \mathrm{cm}^{-1}$ & $\mathrm{~A} / \mathrm{MHz}$ & $\mathrm{B} / \mathrm{MHz}$ & $\lambda_{\text {exc. }} / \AA$ & $\lambda_{\mathrm{fl} .} / \AA$ \\
\hline \multicolumn{6}{|c|}{ New even levels } \\
\hline \multirow[t]{7}{*}{$5 / 2$} & 27993.770 & $754(2)$ & & $5765.513,5932.304$ & 6361.250 \\
\hline & 29389.516 & $749(2)$ & & $5746.61,5842.38$ & 5089.150 \\
\hline & 29806.394 & $779(3)$ & & $5703.42,5920.05$ & $4801.743,5164.812$ \\
\hline & 30123.235 & $657(1)$ & & $5811.02,5920.20$ & $4905.908,5081.628$ \\
\hline & 30590.721 & $622(2)$ & & $5760.72,5891.36$ & 4988.161 \\
\hline & 30963.451 & $661(2)$ & & $5764.73,5881.14$ & 4873.488 \\
\hline & 31299.695 & $800(3)$ & & $5767.06,5781.93$ & 4794.892 \\
\hline \multirow[t]{6}{*}{$7 / 2$} & 28412.971 & $1025(3)$ & & $5720.439,5788.313$ & $4958.234,5629.412$ \\
\hline & 29468.524 & $623(2)$ & & $5739.401,5758.885$ & $5313.584,6005.252$ \\
\hline & 30461.496 & $692(3)$ & & $5667.216,5843.786$ & 4787.551 \\
\hline & 31005.715 & $692(2)$ & & $5750.72,5866.55$ & $4665.944,5080.914$ \\
\hline & 32070.559 & $668(2)$ & & $5700.92,5881.09$ & $4820.053,5995.548$ \\
\hline & 32166.080 & $736(2)$ & & $5670.03,5695.74$ & 4797.956 \\
\hline \multirow[t]{14}{*}{$9 / 2$} & 24706.503 & $975(2)$ & $-5(5)$ & $4285.139,6012.973$ & 6074.993 \\
\hline & 28883.730 & $835(2)$ & & $5719.002,5938.79$ & $5178.856,5570.391$ \\
\hline & 28936.490 & $615(2)$ & & $5701.793,7082.750$ & $5554.061,5675.712$ \\
\hline & 29340.605 & $530(2)$ & & 6087.97, 6207.92 & $4875.446,5059.117$ \\
\hline & 29523.953 & $647(2)$ & & $5796.830,5985.32$ & 5012.608 \\
\hline & 29698.580 & $629(2)$ & & $5664.587,5714.56$ & 5193.739 \\
\hline & 29766.734 & $699(2)$ & & $5899.57,5919.355$ & 4952.322 \\
\hline & 29980.012 & $672(2)$ & & $5826.24,5859.80$ & 5624.088 \\
\hline & 30232.222 & $737(2)$ & & $5741.84,5925.44$ & 4840.699 \\
\hline & 30356.677 & $788(2)$ & & $5733.22,5882.05$ & 4811.703 \\
\hline & 30447.257 & $695(2)$ & & $4999.286,5703.59$ & $4790.816,6081.770$ \\
\hline & 30625.599 & $643(1)$ & $25(50)$ & $5788.26,5790.43$ & $3264.301,4750.218$ \\
\hline & 31677.736 & $433(2)$ & & $5728.571,5831.55$ & 4913.107 \\
\hline & 32402.860 & $544(2)$ & & $5768.327,5878.396$ & $4553.938,4744.046$ \\
\hline \multirow[t]{10}{*}{$11 / 2$} & 28889.260 & $639(1)$ & $40(50)$ & $5693.32,5826.37$ & 5177.373 \\
\hline & 29071.100 & $635(2)$ & & $5765.268,5933.423$ & 6173.640 \\
\hline & 29128.761 & $544(2)$ & & $5746.121,5751.336$ & 5340.960 \\
\hline & 29377.729 & $683(3)$ & & $5665.093,5751.95$ & 5049.631 \\
\hline & 29718.506 & $660(1)$ & & $5708.06,5713.87$ & $4964.182,5177.821$ \\
\hline & 31010.172 & $705(2)$ & & $5875.80,5878.00$ & 5079.762 \\
\hline & 31664.262 & $601(2)$ & & $5733.00,5827.91$ & $4916.362,5903.148$ \\
\hline & 31771.680 & $718(2)$ & & $5799.77,5865.94$ & 5055.597 \\
\hline & 31900.665 & $525(2)$ & & $5748.69,5821.88$ & 5385.041 \\
\hline & 32373.816 & $660(2)$ & & $5724.69,5871.61$ & 4906.194 \\
\hline \multirow[t]{5}{*}{$13 / 2$} & 19654.709 & $800(2)$ & & 5947.91 & 5469.506 \\
\hline & 29051.938 & $540(2)$ & & $5776.87,5799.77$ & $5933.903,5940.178$ \\
\hline & 29111.856 & $581(3)$ & & $5779.674,5919.103$ & 3806.251 \\
\hline & 30600.763 & $656(2)$ & & $6314.88,6586.72$ & $5439.581,5640.794$ \\
\hline & 32623.126 & $591(2)$ & & $5678.62,5786.874$ & 5434.186 \\
\hline \multirow[t]{4}{*}{$15 / 2$} & 27265.604 & $738(3)$ & & $5931.364,6470.303$ & 4368.535 \\
\hline & 31402.846 & $631(2)$ & & $5918.099,6166.705$ & 6166.957 \\
\hline & 32336.849 & $475(2)$ & & $5690.809,5911.88$ & 5884.389 \\
\hline & 33098.154 & $480(2)$ & & $5648.44,5657.19$ & $5454.430,5506.798$ \\
\hline \multirow[t]{2}{*}{$17 / 2$} & 30826.745 & $580(2)$ & & $5608.63,5791.715$ & $5244.434,5590.891$ \\
\hline & 30848.885 & $507(1)$ & & $5690.43,6385.226$ & $5238.350,5583.974$ \\
\hline
\end{tabular}


Table 3: Continued.

\begin{tabular}{|c|c|c|c|c|c|}
\hline$J$ & Energy $/ \mathrm{cm}^{-1}$ & $\mathrm{~A} / \mathrm{MHz}$ & $\mathrm{B} / \mathrm{MHz}$ & $\lambda_{\text {exc. }} / \AA$ & $\lambda_{\mathrm{fl} .} / \AA$ \\
\hline \multirow[t]{2}{*}{$19 / 2$} & 29309.810 & $434(1)$ & & $5697.855,5746.624$ & $6236.808,6889.914$ \\
\hline & 30466.597 & $403(3)$ & & $5705.81,5789.467$ & 6199.288 \\
\hline New odd levels & & & & & 85 \\
\hline \multirow[t]{3}{*}{$3 / 2$} & 29190.205 & $553(1)$ & $0(10)$ & $5698.408,6350.60$ & $5132.144,5444.773$ \\
\hline & 29804.574 & $1115(4)$ & & $5916.390,8035.004$ & $5347.001,5420.660$ \\
\hline & 31343.998 & $1123(3)$ & & $5715.42,5833.47$ & $4422.278,4621.188$ \\
\hline \multirow[t]{6}{*}{$5 / 2$} & 28800.063 & $1057(2)$ & & $5704.28,5769.52$ & 5220.453 \\
\hline & 28920.891 & $1012(3)$ & & $5665.220,5693.46$ & 4465.960 \\
\hline & 29075.845 & $829(2)$ & & $5743.208,5871.61$ & 4435.258 \\
\hline & 30304.145 & $818(2)$ & & $5661.86,5919.58$ & $5277.699,5477.134$ \\
\hline & 30679.627 & $933(2)$ & & $5695.81,5721.43$ & 4766.274 \\
\hline & 30763.323 & $981(2)$ & & $5668.78,5694.15$ & 4538.864 \\
\hline \multirow[t]{14}{*}{$7 / 2$} & 24899.621 & $961(2)$ & & 5920.24 & 4884.455 \\
\hline & 25172.325 & $708(2)$ & & $5826.151,5831.653$ & 5364.250 \\
\hline & 26371.971 & $997(3)$ & & $6549.43,6582.51$ & $5039.832,5330.562$ \\
\hline & 27096.093 & $918(3)$ & & $6470.79,6498.944$ & $5132.395,5238.807$ \\
\hline & 28204.331 & $781(5)$ & & $6467.24,7764.48$ & $4205.426,4466.734$ \\
\hline & 28339.709 & $798(2)$ & & $5709.230,5739.15$ & 4918.284 \\
\hline & 28525.358 & $843(2)$ & & $5739.705,5795.12$ & $4403.570,5453.846$ \\
\hline & 28859.879 & $538(2)$ & & $5749.67,5807.763$ & $4339.625,5060.635$ \\
\hline & 28978.464 & $1047(3)$ & & $5736.02,5768.02$ & 4839.335 \\
\hline & 29579.960 & $720(2)$ & & $5702.76,5792.82$ & 4338.231 \\
\hline & 29675.405 & $865(1)$ & & $5671.88,5681.28$ & 5005.949 \\
\hline & 30975.616 & $734(2)$ & & $5703.705,5843.45$ & $5031.837,5051.337$ \\
\hline & 31668.068 & $852(2)$ & & $5673.09,5747.12$ & $4969.170,5100.550$ \\
\hline & 34243.714 & $655(2)$ & & 5763.922 & $3608.018,4734.423$ \\
\hline \multirow[t]{13}{*}{$9 / 2$} & 25634.179 & $674(1)$ & & $5673.44,5774.092$ & 4715.227 \\
\hline & 26337.481 & $810(1)$ & & $7736.76,7935.73$ & $4563.835,4873.218$ \\
\hline & 26718.738 & $826(2)$ & & $5729.066,5875.89$ & 4997.468 \\
\hline & 27394.138 & $792(3)$ & & $6470.73,6522.91$ & $4742.301,4792.854$ \\
\hline & 27745.272 & $862(2)$ & & $5696.259,5749.322$ & $4753.538,5363.244$ \\
\hline & 28579.596 & $765(2)$ & $40(50)$ & $5661.189,5747.116$ & $5014.710,5061.743$ \\
\hline & 29149.204 & $730(1)$ & & $4377.825,5785.463$ & 5028.688 \\
\hline & 30035.792 & $884(2)$ & & $5707.45,5739.56$ & $4459.377,4813.971$ \\
\hline & 30896.170 & $733(2)$ & & $5781.92,5841.280$ & $3987.182,4371.907$ \\
\hline & 30901.231 & $730(2)$ & & $5780.229,5839.55$ & $4370.938,4491.623$ \\
\hline & 31447.902 & $665(2)$ & & $5744.87,5887.789$ & 4866.273 \\
\hline & 31528.624 & $746(2)$ & & $5789.76,5793.565$ & 5531.149 \\
\hline & 33687.796 & $744(2)$ & & $5691.05,5764.22$ & 3468.665 \\
\hline \multirow[t]{12}{*}{$11 / 2$} & 25900.352 & $858(2)$ & & $5686.67,5856.16$ & 4656.763 \\
\hline & 26159.533 & $672(3)$ & & $5707.577,5861.933$ & 4915.860 \\
\hline & 29195.032 & $805(2)$ & & $5718.648,5894.447$ & $4705.958,5581.246$ \\
\hline & 29614.075 & $900(5)$ & & $5697.63,5714.00$ & $4614.926,4966.186$ \\
\hline & 29985.372 & $775(2)$ & & $5799.086,7848.97$ & $5222.124,5239.264$ \\
\hline & 30053.346 & $759(2)$ & & $5701.741,5747.20$ & $5052.454,5220.665$ \\
\hline & 30094.834 & $825(2)$ & & $5733.53,5762.500$ & $5314.286,5394.551$ \\
\hline & 30111.020 & $786(2)$ & & $5728.21,5757.13$ & $4252.777,4272.879$ \\
\hline & 30347.477 & $700(3)$ & & $6430.416,6470.81$ & $4836.005,5029.500$ \\
\hline & 31895.205 & $668(2)$ & & $5910.73,5938.70$ & 3698.720 \\
\hline & 32289.455 & $665(1)$ & & $5716.368,5806.88$ & 5458.571 \\
\hline & 32513.960 & $655(2)$ & & $5643.92,5786.83$ & $4535.186,4749.025$ \\
\hline
\end{tabular}


Table 3: Continued.

\begin{tabular}{|c|c|c|c|c|c|}
\hline$J$ & Energy $/ \mathrm{cm}^{-1}$ & $\mathrm{~A} / \mathrm{MHz}$ & $\mathrm{B} / \mathrm{MHz}$ & $\lambda_{\text {exc. }} / \AA$ & $\lambda_{\mathrm{fl} .} / \AA$ \\
\hline \multirow[t]{15}{*}{$13 / 2$} & 24781.116 & $858(2)$ & $-20(10)$ & $5828.953,5940.199$ & 5588.731 \\
\hline & 28487.636 & $672(2)$ & & $5685.54,6561.17$ & $4967.870,5201.762$ \\
\hline & 28851.007 & $584(2)$ & & $5690.540,5756.26$ & 5437.904 \\
\hline & 28921.386 & $697(2)$ & & $5726.28,5733.026$ & 4959.956 \\
\hline & 29663.117 & $651(3)$ & & $5698.02,5736.137$ & 4693.694 \\
\hline & 29774.999 & $615(2)$ & & $5661.92,5704.72$ & 4758.430 \\
\hline & 29836.950 & $535(2)$ & & $5679.492,5684.625$ & 5388.150 \\
\hline & 30284.567 & $681(2)$ & & $5671.81,5719.21$ & $4994.092,5317.344$ \\
\hline & 30412.010 & $658(2)$ & & $5825.433,5868.635$ & $5442.042,5499.812$ \\
\hline & 30562.652 & $537(1)$ & & $5703.92,5740.23$ & $4742.700,4964.105$ \\
\hline & 30644.652 & $628(2)$ & & $5789.57,5810.44$ & 4943.974 \\
\hline & 31005.761 & $586(2)$ & & $5761.69,5834.90$ & $4867.409,5141.807$ \\
\hline & 32608.542 & $620(3)$ & & $5701.20,5800.11$ & $3844.331,4515.808$ \\
\hline & 33052.848 & $555(2)$ & & $5785.33,5860.70$ & 3982.695 \\
\hline & 33932.700 & $737(1)$ & & $5667.90,5709.27$ & $5165.634,5260.914$ \\
\hline \multirow[t]{13}{*}{$15 / 2$} & 26332.040 & $506(2)$ & $-45(5)$ & $5926.883,5691.075$ & 5345.561 \\
\hline & 27294.906 & $790(5)$ & & $6314.57,7709.96$ & 5280.870 \\
\hline & 27950.530 & $824(1)$ & & $5703.941,6314.40$ & $5210.956,5407.968$ \\
\hline & 28754.446 & $639(2)$ & & $5781.568,5788.44$ & 5466.620 \\
\hline & 29094.822 & $634(2)$ & & $5702.23,5751.989$ & 4728.263 \\
\hline & 29942.851 & $583(2)$ & & $5650.598,5810.239$ & $4949.732,5150.310$ \\
\hline & 29989.064 & $528(2)$ & & $5794.67,5817.55$ & 5570.287 \\
\hline & 30788.090 & $528(2)$ & & $5762.395,5834.890$ & 4919.547 \\
\hline & 31039.972 & $553(1)$ & & $5689.099,5750.352$ & $4091.104,4860.176$ \\
\hline & 31668.549 & $544(2)$ & & $5729.96,5919.86$ & 5625.687 \\
\hline & 31817.886 & $437(2)$ & & $5716.08,5762.81$ & $4911.429,4916.388$ \\
\hline & 32672.439 & $601(2)$ & & $5680.510,5778.688$ & $5014.698,5031.823$ \\
\hline & 32849.815 & $540(2)$ & & $5720.040,5735.74$ & $4467.124,5623.828$ \\
\hline \multirow[t]{8}{*}{$17 / 2$} & 25909.173 & $854(2)$ & $-45(5)$ & $5697.960,5831.453$ & $5831.453,5697.960$ \\
\hline & 27844.889 & $602(2)$ & $-40(50)$ & $5752.739,5774.425$ & 5239.809 \\
\hline & 29622.951 & $501(1)$ & $15(20)$ & $5754.650,5920.309$ & $5035.700,5412.236$ \\
\hline & 29899.954 & $529(1)$ & & $5664.331,5847.876$ & $5161.717,6143.342$ \\
\hline & 30346.200 & $518(1)$ & & $5699.110,5913.005$ & $5913.005,5699.110$ \\
\hline & 31270.847 & $512(2)$ & & $5798.136,5863.62$ & $4820.511,4968.937$ \\
\hline & 33305.483 & $450(2)$ & & $6404.22,6470.68$ & $5775.171,6231.393$ \\
\hline & 35453.400 & $595(1)$ & & $5820.62,5848.02$ & $3888.734,5629.604$ \\
\hline $19 / 2$ & 29950.715 & $418(2)$ & & $5783.441,5807.582$ & 5482.025 \\
\hline \multirow[t]{2}{*}{$21 / 2$} & 30543.982 & $392(2)$ & & $5909.466,6801.441$ & $5155.184,5591.534$ \\
\hline & 32741.057 & $332(2)$ & & 5917.00 & 5230.205 \\
\hline
\end{tabular}

was known, and none of the combining levels could be identified by $J$ - and $A$-values (Figure 1(a)). If one of the fluorescence lines was in the wavelength range of our laser light sources, we tried to perform a second excitation at this wavelength. With some luck, we were able to identify the lower level of the new excitation. Then the energy of the unknown upper level could be determined, and in sequence, using the wave number of the first excitation, the energy of the new lower level (Figure 1(b)). If none of the fluorescence lines could be reached with our available lasers, we had to choose the following procedure: if there were more than two fluorescence lines whose wavelengths could be determined accurately, we had a chance to find the positions of the levels by explaining the wave number differences of the fluorescence lines as differences between the energies of already known levels (Figure 1(c)). Of course, we had to consider the ladders of even and odd levels separately, since we did not know the parity of the upper unknown level.

In 2007, we were able to record a highly resolved FT emission spectrum, which contains more than 25000 spectral lines, using our hollow cathode discharge as light source. A first analysis led to the classification of about 1200 lines as 
TABLE 4: Spectral lines explained by the new even levels.

\begin{tabular}{|c|c|c|c|c|c|c|c|c|c|c|c|}
\hline \multirow[b]{2}{*}{$J$} & \multicolumn{3}{|c|}{ New upper even level } & \multicolumn{3}{|c|}{ Line } & \multicolumn{5}{|c|}{ Combining known lower odd level } \\
\hline & Energy $/ \mathrm{cm}^{-1}$ & $\mathrm{~A} / \mathrm{MHz}$ & $\mathrm{B} / \mathrm{MHz}$ & $\lambda / \AA$ & C. & SNR & $J$ & Energy $/ \mathrm{cm}^{-1}$ & $\mathrm{~A} / \mathrm{MHz}$ & $\mathrm{B} / \mathrm{MHz}$ & Reference to col. 10,11 \\
\hline \multirow[t]{3}{*}{$13 / 2$} & 19654.709 & $800(2)$ & & 5469.506 & $\mathrm{f}$ & 190 & $11 / 2$ & 1376.602 & 730.393 & -11.877 & {$[7]$} \\
\hline & & & & 5947.91 & nl, e & 5 & $13 / 2$ & 2846.741 & 613.240 & -12.850 & [7] \\
\hline & & & & 6545.421 & & 30 & $15 / 2$ & 4381.072 & 541.575 & -14.558 & [7] \\
\hline \multirow[t]{4}{*}{$9 / 2$} & 24706.503 & $975(2)$ & & 4285.139 & $\mathrm{nl}, \mathrm{e}$ & 11 & $11 / 2$ & 1376.602 & 730.393 & -11.877 & [7] \\
\hline & & & & 6012.973 & $\mathrm{nl}, \mathrm{e}$ & 12 & $11 / 2$ & 8080.402 & 238.352 & -22.961 & [7] \\
\hline & & & & 6074.993 & $\mathrm{f}$ & 135 & $9 / 2$ & 8250.141 & 213.531 & -4.136 & [7] \\
\hline & & & & 7003.424 & & 16 & $11 / 2$ & 10431.716 & $701.7(5)$ & $-10(7)$ & [9] \\
\hline \multirow[t]{8}{*}{$15 / 2$} & 27265.604 & $738(3)$ & & 4368.535 & $\mathrm{nl}, \mathrm{f}$ & 8 & $15 / 2$ & 4381.072 & 541.575 & -14.558 & [7] \\
\hline & & & & 5394.523 & $\mathrm{nl}$ & 11 & $13 / 2$ & 8733.440 & 854.297 & -31.807 & [7] \\
\hline & & & & 5931.364 & $\mathrm{e}$ & 17 & $13 / 2$ & 10410.745 & $655.9(3)$ & $-29(7)$ & [8] \\
\hline & & & & 6023.642 & $\mathrm{f}$ & 70 & $15 / 2$ & 10668.950 & 951.310 & -2.670 & [7] \\
\hline & & & & 6441.818 & & 55 & $13 / 2$ & 11746.328 & $401(1)$ & $50(20)$ & [19] \\
\hline & & & & 6470.303 & $\mathrm{nl}, \mathrm{e}$ & 12 & $15 / 2$ & 11814.647 & $355(2)$ & $0(10)$ & [19] \\
\hline & & & & 6645.548 & & 24 & $13 / 2$ & 12222.091 & $1133(4)$ & - & This work \\
\hline & & & & 7837.664 & $\mathrm{nl}$ & 4 & $13 / 2$ & 14510.207 & $1085(2)$ & - & This work \\
\hline \multirow[t]{6}{*}{$5 / 2$} & 27993.770 & $754(2)$ & & 5765.513 & $\mathrm{e}$ & 20 & $7 / 2$ & 10654.070 & $169(2)$ & - & [19] \\
\hline & & & & 5932.304 & e & 25 & $5 / 2$ & 11141.576 & $169(2)$ & - & [19] \\
\hline & & & & 6025.783 & $\mathrm{nl}$ & 8 & $7 / 2$ & 11403.011 & $1142(3)$ & - & [19] \\
\hline & & & & 6293.545 & $\mathrm{nl}$ & 5 & $5 / 2$ & 12108.867 & $1275(2)$ & - & [19] \\
\hline & & & & 6361.250 & $\mathrm{nl}$ & 15 & $7 / 2$ & 12277.935 & $1760(4)$ & - & [19] \\
\hline & & & & 7148.782 & $\mathrm{nl}$ & 4 & $7 / 2$ & 14009.225 & $1100(1)$ & - & [19] \\
\hline \multirow[t]{6}{*}{$7 / 2$} & 28412.971 & $1025(3)$ & & 3518.513 & $\mathrm{nl}$ & 16 & $9 / 2$ & 0.000 & 926.209 & -11.878 & {$[7]$} \\
\hline & & & & 4958.234 & $\mathrm{nl}, \mathrm{f}$ & 6 & $9 / 2$ & 8250.141 & 213.531 & -4.136 & [7] \\
\hline & & & & 5629.412 & $\mathrm{f}$ & 45 & $7 / 2$ & 10654.070 & $169(2)$ & - & [19] \\
\hline & & & & 5720.439 & e & 90 & $9 / 2$ & 10936.652 & $930(1)$ & $0(10)$ & [19] \\
\hline & & & & 5788.313 & e & 75 & $5 / 2$ & 11141.576 & $169(2)$ & - & [19] \\
\hline & & & & 5877.279 & & 18 & $7 / 2$ & 11403.011 & $1142(3)$ & - & [19] \\
\hline \multirow[t]{8}{*}{$9 / 2$} & 28883.730 & $835(2)$ & & 5178.856 & $\mathrm{nl}, \mathrm{f}$ & 14 & $9 / 2$ & 9579.820 & $789(1)$ & - & [19] \\
\hline & & & & 5570.391 & $\mathrm{nl}, \mathrm{f}$ & 25 & $9 / 2$ & 10936.652 & $930(1)$ & $0(10)$ & [19] \\
\hline & & & & 5719.002 & $\mathrm{e}$ & 48 & $7 / 2$ & 11403.011 & $1142(3)$ & - & [19] \\
\hline & & & & 5938.79 & $\mathrm{nl}, \mathrm{e}$ & 1 & $9 / 2$ & 12049.942 & $275(2)$ & - & [19] \\
\hline & & & & 6020.327 & $\mathrm{nl}, \mathrm{f}$ & 1 & $7 / 2$ & 12277.935 & $1760(4)$ & - & [19] \\
\hline & & & & 6223.887 & $\mathrm{nl}, \mathrm{f}$ & 6 & $9 / 2$ & 12821.044 & $1127(3)$ & - & [19] \\
\hline & & & & 6262.198 & $\mathrm{nl}, \mathrm{f}$ & 12 & $7 / 2$ & 12919.316 & $180(2)$ & - & [19] \\
\hline & & & & 6440.183 & $\mathrm{nl}, \mathrm{f}$ & 1 & $11 / 2$ & 13360.511 & $151(3)$ & - & [19] \\
\hline \multirow[t]{7}{*}{$11 / 2$} & 28889.260 & $639(1)$ & $40(50)$ & 5177.373 & $\mathrm{f}$ & 220 & $9 / 2$ & 9579.820 & $789(1)$ & - & [19] \\
\hline & & & & 5690.96 & $\mathrm{nl}, \mathrm{e}$ & 1 & $11 / 2$ & 11322.443 & $1272(1)$ & $75(50)$ & [19] \\
\hline & & & & 5693.32 & $\mathrm{nl}, \mathrm{e}$ & 1 & $9 / 2$ & 11329.696 & $530(3)$ & - & [19] \\
\hline & & & & 5826.37 & $\mathrm{nl}, \mathrm{e}$ & 1 & $9 / 2$ & 11730.668 & $1365(5)$ & - & [19] \\
\hline & & & & 5831.69 & $\mathrm{nl}, \mathrm{e}$ & 1 & $13 / 2$ & 11746.328 & $401(1)$ & $50(20)$ & [19] \\
\hline & & & & 5918.27 & $\mathrm{nl}, \mathrm{e}$ & 1 & $11 / 2$ & 11997.137 & $585(3)$ & - & [19] \\
\hline & & & & 6817.989 & $\mathrm{nl}$ & 4 & $11 / 2$ & 14226.220 & $869(3)$ & - & [19] \\
\hline \multirow[t]{6}{*}{$9 / 2$} & 28936.490 & $615(2)$ & & 5554.061 & $\mathrm{nl}, \mathrm{f}$ & 12 & $9 / 2$ & 10936.652 & $930(1)$ & $0(10)$ & [19] \\
\hline & & & & 5675.712 & $\mathrm{nl}, \mathrm{f}$ & 1 & $11 / 2$ & 11322.443 & $1272(1)$ & $75(50)$ & [19] \\
\hline & & & & 5701.793 & $\mathrm{nl}, \mathrm{e}, \mathrm{f}$ & 16 & $7 / 2$ & 11403.011 & $1142(3)$ & - & [19] \\
\hline & & & & 5920.23 & $\mathrm{nl}, \mathrm{e}$ & 1 & $9 / 2$ & 12049.942 & $275(2)$ & - & [19] \\
\hline & & & & 6203.512 & $\mathrm{f}$ & 13 & $9 / 2$ & 12821.044 & $1127(3)$ & - & [19] \\
\hline & & & & 6225.390 & $\mathrm{nl}, \mathrm{f}$ & 1 & $11 / 2$ & 12877.682 & 1139 (2) & - & [19] \\
\hline
\end{tabular}


Table 4: Continued.

\begin{tabular}{|c|c|c|c|c|c|c|c|c|c|c|c|}
\hline \multicolumn{4}{|c|}{ New upper even level } & \multicolumn{3}{|c|}{ Line } & \multicolumn{5}{|c|}{ Combining known lower odd level } \\
\hline \multirow[t]{4}{*}{$J$} & Energy/ $\mathrm{cm}^{-1}$ & $\mathrm{~A} / \mathrm{MHz}$ & $\mathrm{B} / \mathrm{MHz}$ & $\lambda / \AA$ & C. & SNR & $J$ & Energy $/ \mathrm{cm}^{-1}$ & $\mathrm{~A} / \mathrm{MHz}$ & $\mathrm{B} / \mathrm{MHz}$ & Reference to col. 10, 11 \\
\hline & & & & 6694.189 & $\mathrm{f}$ & 23 & $11 / 2$ & 14002.294 & $566(1)$ & - & {$[19]$} \\
\hline & & & & & & & & & & $-125(50)$ & \\
\hline & & & & 7082.750 & $e, f$ & 34 & $11 / 2$ & 14821.565 & $544(2)$ & & This work \\
\hline \multirow[t]{7}{*}{$13 / 2$} & 29051.938 & $540(2)$ & & 3612.294 & $\mathrm{nl}$ & 3 & $11 / 2$ & 1376.602 & 730.393 & -11.877 & [7] \\
\hline & & & & 3814.953 & $\mathrm{nl}$ & 4 & $13 / 2$ & 2846.741 & 613.240 & -12.850 & {$[7]$} \\
\hline & & & & 5776.87 & $\mathrm{nl}, \mathrm{e}$ & 8 & $13 / 2$ & 11746.328 & $401(1)$ & $50(20)$ & [19] \\
\hline & & & & 5799.77 & $\mathrm{nl}, \mathrm{e}$ & 1 & $15 / 2$ & 11814.647 & $355(2)$ & $0(10)$ & [19] \\
\hline & & & & 5933.903 & $\mathrm{nl}, \mathrm{f}$ & 38 & $11 / 2$ & 12204.286 & $1010(2)$ & - & This work \\
\hline & & & & 5940.178 & $\mathrm{f}$ & 35 & $13 / 2$ & 12222.091 & $1133(4)$ & - & This work \\
\hline & & & & 6455.473 & $\mathrm{nl}, \mathrm{e}$ & 10 & $15 / 2$ & 13565.490 & $917(1)$ & $10(10)$ & [19] \\
\hline \multirow[t]{7}{*}{$11 / 2$} & 29071.100 & $635(2)$ & & 5765.268 & nl, e & 5 & $9 / 2$ & 11730.668 & $1365(5)$ & - & [19] \\
\hline & & & & 5873.411 & $\mathrm{nl}, \mathrm{e}$ & 5 & $9 / 2$ & 12049.942 & $275(2)$ & - & [19] \\
\hline & & & & 5927.159 & $\mathrm{nl}$ & 6 & $11 / 2$ & 12204.286 & $1010(2)$ & - & This work \\
\hline & & & & 5933.423 & $\mathrm{nl}, \mathrm{e}$ & 30 & $13 / 2$ & 12222.091 & $1133(4)$ & - & This work \\
\hline & & & & 6152.120 & $\mathrm{nl}$ & 6 & $9 / 2$ & 12821.044 & $1127(3)$ & - & [19] \\
\hline & & & & 6173.640 & $\mathrm{nl}, \mathrm{f}$ & 40 & $11 / 2$ & 12877.682 & $1139(2)$ & - & [19] \\
\hline & & & & 6360.753 & & 12 & $9 / 2$ & 13354.043 & $1272(3)$ & - & [19] \\
\hline \multirow[t]{6}{*}{$13 / 2$} & 29111.856 & $581(3)$ & & 3604.491 & $\mathrm{nl}$ & 4 & $11 / 2$ & 1376.602 & 730.393 & -11.877 & {$[7]$} \\
\hline & & & & 3806.251 & $\mathrm{nl}, \mathrm{f}$ & 10 & $13 / 2$ & 2846.741 & 613.240 & -12.850 & [7] \\
\hline & & & & 5420.630 & $\mathrm{nl}$ & 15 & $15 / 2$ & 10668.950 & 951.310 & -2.670 & [7] \\
\hline & & & & 5779.674 & $\mathrm{nl}, \mathrm{e}$ & 5 & $15 / 2$ & 11814.647 & $355(2)$ & $0(10)$ & [19] \\
\hline & & & & 5919.103 & $\mathrm{nl}, \mathrm{e}$ & 15 & $13 / 2$ & 12222.091 & $1133(4)$ & - & This work \\
\hline & & & & 6158.141 & $\mathrm{f}$ & 19 & $11 / 2$ & 12877.682 & $1139(2)$ & - & [19] \\
\hline \multirow[t]{5}{*}{$11 / 2$} & 29128.761 & $544(2)$ & & 3432.049 & $\mathrm{nl}$ & 26 & $9 / 2$ & 0.000 & 926.209 & -11.878 & {$[7]$} \\
\hline & & & & 5340.960 & $\mathrm{f}$ & 37 & $13 / 2$ & 10410.745 & $655.9(3)$ & $-29(7)$ & {$[8]$} \\
\hline & & & & 5746.121 & $\mathrm{nl}, \mathrm{e}$ & 22 & $9 / 2$ & 11730.668 & $1365(5)$ & - & [19] \\
\hline & & & & 5751.336 & $\mathrm{nl}, \mathrm{e}$ & 6 & $13 / 2$ & 11746.328 & $401(1)$ & $50(20)$ & [19] \\
\hline & & & & 5853.578 & $\mathrm{nl}$ & 5 & $9 / 2$ & 12049.942 & $275(2)$ & - & [19] \\
\hline \multirow[t]{6}{*}{$19 / 2$} & 29309.810 & $434(1)$ & & 5697.855 & $\mathrm{e}$ & 5 & $17 / 2$ & 11764.216 & $892.5(7)$ & $-10(25)$ & {$[9]$} \\
\hline & & & & 5746.624 & e & 55 & $19 / 2$ & 11913.115 & 829.9 (2) & $2(8)$ & {$[9]$} \\
\hline & & & & 6236.808 & $\mathrm{f}$ & 105 & $17 / 2$ & 13280.404 & $208(2)$ & $20(50)$ & [19] \\
\hline & & & & 6889.914 & $\mathrm{f}$ & 23 & $19 / 2$ & 14799.842 & $126(5)$ & - & [19] \\
\hline & & & & 8593.623 & & 5 & $17 / 2$ & 17676.472 & $856(2)$ & - & This work \\
\hline & & & & 9477.685 & & 12 & $19 / 2$ & 18761.608 & $870(1)$ & - & This work \\
\hline \multirow[t]{7}{*}{$9 / 2$} & 29340.605 & $530(2)$ & & 4875.446 & $\mathrm{nl}, \mathrm{f}$ & 1 & $11 / 2$ & 8835.389 & 949.091 & -13.721 & [7] \\
\hline & & & & 5059.117 & $\mathrm{f}$ & 11 & $9 / 2$ & 9579.820 & 789 (1) & - & [19] \\
\hline & & & & 5548.412 & $\mathrm{nl}, \mathrm{f}$ & 18 & $11 / 2$ & 11322.443 & $1272(1)$ & $75(50)$ & [19] \\
\hline & & & & 5833.94 & $\mathrm{nl}$ & 1 & $11 / 2$ & 12204.286 & $1010(2)$ & - & This work \\
\hline & & & & 6087.97 & $\mathrm{nl}, \mathrm{e}$ & 1 & $7 / 2$ & 12919.316 & $180(2)$ & - & [19] \\
\hline & & & & 6207.92 & $\mathrm{nl}, \mathrm{e}$ & 1 & $7 / 2$ & 13236.606 & $726(1)$ & - & [19] \\
\hline & & & & 6614.387 & $\mathrm{nl}, \mathrm{e}$ & 1 & $11 / 2$ & 14226.220 & 869 (3) & - & [19] \\
\hline \multirow[t]{5}{*}{$11 / 2$} & 29377.729 & $683(3)$ & & 5049.631 & $\mathrm{f}$ & 120 & $9 / 2$ & 9579.820 & 789 (1) & - & [19] \\
\hline & & & & 5276.688 & $\mathrm{nl}$ & 8 & $11 / 2$ & 10431.716 & $701.7(5)$ & $-10(7)$ & {$[9]$} \\
\hline & & & & 5665.093 & $\mathrm{nl}, \mathrm{e}$ & 15 & $9 / 2$ & 11730.668 & $1365(5)$ & - & [19] \\
\hline & & & & 5751.95 & $\mathrm{nl}, \mathrm{e}$ & 1 & $11 / 2$ & 11997.137 & $585(3)$ & - & [19] \\
\hline & & & & 6239.035 & $\mathrm{nl}$ & 5 & $9 / 2$ & 13354.043 & $1272(3)$ & - & [19] \\
\hline
\end{tabular}


TABle 4: Continued.

\begin{tabular}{|c|c|c|c|c|c|c|c|c|c|c|c|}
\hline \multicolumn{4}{|c|}{ New upper even level } & \multicolumn{3}{|c|}{ Line } & \multicolumn{5}{|c|}{ Combining known lower odd level } \\
\hline$J$ & Energy $/ \mathrm{cm}^{-1}$ & $\mathrm{~A} / \mathrm{MHz}$ & $\mathrm{B} / \mathrm{MHz}$ & $\lambda / \AA$ & C. & SNR & $J$ & Energy $/ \mathrm{cm}^{-1}$ & $\mathrm{~A} / \mathrm{MHz}$ & $\mathrm{B} / \mathrm{MHz}$ & Reference to col. 10,11 \\
\hline \multirow[t]{4}{*}{$5 / 2$} & 29389.516 & $749(2)$ & & 4899.854 & $\mathrm{nl}$ & 10 & $3 / 2$ & 8986.443 & $1029(3)$ & - & This work \\
\hline & & & & 5089.150 & $\mathrm{f}$ & 30 & $5 / 2$ & 9745.334 & $626(1)$ & $5(3)$ & [4] \\
\hline & & & & 5746.61 & $\mathrm{nl}, \mathrm{e}$ & 1 & $3 / 2$ & 11992.788 & $225(5)$ & - & This work \\
\hline & & & & 5842.38 & $\mathrm{nl}$, e & 1 & $7 / 2$ & 12277.935 & $1760(4)$ & - & [19] \\
\hline \multirow[t]{8}{*}{$7 / 2$} & 29468.524 & $623(2)$ & & 5313.584 & $\mathrm{nl}, \mathrm{f}$ & 1 & $7 / 2$ & 10654.070 & $169(2)$ & - & This work \\
\hline & & & & 5394.610 & $\mathrm{nl}$ & 10 & $9 / 2$ & 10936.652 & $930(1)$ & $0(10)$ & [19] \\
\hline & & & & 5511.504 & $\mathrm{nl}$ & 10 & $9 / 2$ & 11329.696 & $530(3)$ & - & [19] \\
\hline & & & & 5739.401 & $\mathrm{e}$ & 45 & $9 / 2$ & 12049.942 & $275(2)$ & - & [19] \\
\hline & & & & 5758.885 & $\mathrm{nl}$, e & 4 & $5 / 2$ & 12108.867 & $1275(2)$ & - & [19] \\
\hline & & & & 6005.252 & $\mathrm{nl}, \mathrm{f}$ & 5 & $9 / 2$ & 12821.044 & $1127(3)$ & - & [19] \\
\hline & & & & 6040.912 & $\mathrm{nl}, \mathrm{f}$ & 1 & $7 / 2$ & 12919.316 & $180(2)$ & - & [19] \\
\hline & & & & 6308.548 & $\mathrm{nl}, \mathrm{f}$ & 1 & $7 / 2$ & 13621.400 & $879(1)$ & $-10(10)$ & This work \\
\hline \multirow[t]{5}{*}{$9 / 2$} & 29523.953 & $647(2)$ & & 5012.608 & $\mathrm{f}$ & 35 & $9 / 2$ & 9579.820 & 789 (1) & - & [19] \\
\hline & & & & 5772.18 & $\mathrm{nl}, \mathrm{e}$ & 1 & $11 / 2$ & 12204.286 & $1010(2)$ & - & This work \\
\hline & & & & 5796.830 & nl, e & 10 & $7 / 2$ & 12277.935 & $1760(4)$ & - & [19] \\
\hline & & & & 5985.32 & $\mathrm{nl}$, e & 1 & $9 / 2$ & 12821.044 & $1127(3)$ & - & [19] \\
\hline & & & & 6005.688 & & 20 & $11 / 2$ & 12877.682 & $1139(2)$ & - & [19] \\
\hline \multirow[t]{7}{*}{$9 / 2$} & 29698.580 & $629(2)$ & & 5193.739 & $\mathrm{f}$ & 25 & $7 / 2$ & 10449.997 & $541(2)$ & - & [19] \\
\hline & & & & 5664.587 & $\mathrm{e}$ & 15 & $9 / 2$ & 12049.942 & $275(2)$ & - & [19] \\
\hline & & & & 5714.56 & $\mathrm{nl}, \mathrm{e}$ & 1 & $11 / 2$ & 12204.286 & $1010(2)$ & - & This work \\
\hline & & & & 5923.396 & $\mathrm{nl}$ & 35 & $9 / 2$ & 12821.044 & $1127(3)$ & - & [19] \\
\hline & & & & 6118.984 & & 8 & $11 / 2$ & 13360.511 & $151(3)$ & - & [19] \\
\hline & & & & 6218.279 & & 12 & $7 / 2$ & 13621.400 & $879(1)$ & $-10(10)$ & This work \\
\hline & & & & 6719.928 & $\mathrm{nl}$ & 6 & $11 / 2$ & 14821.565 & $544(2)$ & & This work \\
\hline \multirow[t]{7}{*}{$11 / 2$} & 29718.506 & $660(1)$ & & 3363.940 & $\mathrm{nl}$ & 53 & $9 / 2$ & 0.000 & 926.209 & -11.878 & [7] \\
\hline & & & & 4964.182 & $\mathrm{f}$ & 55 & $9 / 2$ & 9579.820 & 789 (1) & - & [19] \\
\hline & & & & 5177.821 & $\mathrm{f}$ & 50 & $13 / 2$ & 10410.745 & 655.9 (3) & $-29(7)$ & {$[8]$} \\
\hline & & & & 5434.435 & $\mathrm{nl}, \mathrm{f}$ & 12 & $11 / 2$ & 11322.443 & $1272(1)$ & $75(50)$ & [19] \\
\hline & & & & 5708.06 & $\mathrm{nl}$, e & 1 & $11 / 2$ & 12204.286 & $1010(2)$ & - & This work \\
\hline & & & & 5713.87 & $\mathrm{nl}, \mathrm{e}$ & 1 & $13 / 2$ & 12222.091 & $1133(4)$ & - & This work \\
\hline & & & & 6118.737 & & 25 & $9 / 2$ & 13379.788 & $932(3)$ & $10(20)$ & [19] \\
\hline \multirow[t]{5}{*}{$9 / 2$} & 29766.734 & 699 (2) & & 3521.355 & $\mathrm{nl}$ & 2 & $11 / 2$ & 1376.602 & 730.393 & -11.877 & [7] \\
\hline & & & & 4952.322 & $\mathrm{f}$ & 15 & $9 / 2$ & 9579.820 & $789(1)$ & - & [19] \\
\hline & & & & 5692.390 & $\mathrm{nl}$ & 6 & $11 / 2$ & 12204.286 & $1010(2)$ & - & This work \\
\hline & & & & 5899.57 & $\mathrm{nl}, \mathrm{e}$ & 1 & $9 / 2$ & 12821.044 & $1127(3)$ & - & [19] \\
\hline & & & & 5919.355 & nl, e & 18 & $11 / 2$ & 12877.682 & $1139(2)$ & - & [19] \\
\hline \multirow[t]{10}{*}{$5 / 2$} & 29806.394 & 779 (3) & & 4801.743 & $\mathrm{f}$ & 18 & $3 / 2$ & 8986.443 & $1029(23)$ & - & This work \\
\hline & & & & 5164.812 & $\mathrm{f}$ & 5 & $7 / 2$ & 10449.997 & $541(2)$ & - & [19] \\
\hline & & & & 5219.846 & $\mathrm{nl}, \mathrm{f}$ & 7 & $7 / 2$ & 10654.070 & $169(2)$ & - & This work \\
\hline & & & & 5356.188 & $\mathrm{f}$ & 15 & $5 / 2$ & 11141.576 & $169(2)$ & - & [19] \\
\hline & & & & 5612.128 & $\mathrm{nl}, \mathrm{f}$ & 1 & $3 / 2$ & 11992.788 & $225(5)$ & - & This work \\
\hline & & & & 5648.942 & $\mathrm{nl}, \mathrm{f}$ & 13 & $5 / 2$ & 12108.867 & $1275(2)$ & - & [19] \\
\hline & & & & 5703.42 & $\mathrm{nl}, \mathrm{e}$ & 1 & $7 / 2$ & 12277.935 & $1760(4)$ & - & [19] \\
\hline & & & & 5920.05 & $\mathrm{nl}, \mathrm{e}$ & 1 & $7 / 2$ & 12919.316 & $180(2)$ & - & [19] \\
\hline & & & & 6310.689 & $\mathrm{nl}, \mathrm{f}$ & 1 & $5 / 2$ & 13964.645 & $185(2)$ & - & [19] \\
\hline & & & & 6328.498 & $\mathrm{nl}, \mathrm{f}$ & 1 & $7 / 2$ & 14009.225 & $1100(1)$ & - & [19] \\
\hline
\end{tabular}


Table 4: Continued.

\begin{tabular}{|c|c|c|c|c|c|c|c|c|c|c|c|}
\hline \multicolumn{4}{|c|}{ New upper even level } & \multicolumn{3}{|c|}{ Line } & \multicolumn{5}{|c|}{ Combining known lower odd level } \\
\hline$J$ & Energy $/ \mathrm{cm}^{-1}$ & $\mathrm{~A} / \mathrm{MHz}$ & $\mathrm{B} / \mathrm{MHz}$ & $\lambda / \AA$ & C. & SNR & $J$ & Energy/ $\mathrm{cm}^{-1}$ & $\mathrm{~A} / \mathrm{MHz}$ & $\mathrm{B} / \mathrm{MHz}$ & Reference to col. 10,11 \\
\hline \multirow[t]{4}{*}{$9 / 2$} & 29980.012 & $672(2)$ & & 4900.548 & & 10 & $9 / 2$ & 9579.820 & $789(1)$ & - & {$[19]$} \\
\hline & & & & 5624.088 & $\mathrm{nl}, \mathrm{f}$ & 1 & $11 / 2$ & 12204.286 & $1010(2)$ & - & This work \\
\hline & & & & 5826.24 & $\mathrm{nl}, \mathrm{e}$ & 1 & $9 / 2$ & 12821.044 & $1127(3)$ & - & [19] \\
\hline & & & & 5859.80 & $\mathrm{nl}, \mathrm{e}$ & 1 & $7 / 2$ & 12919.316 & $180(2)$ & - & [19] \\
\hline \multirow[t]{8}{*}{$5 / 2$} & 30123.235 & $657(1)$ & & 4729.763 & & 12 & $3 / 2$ & 8986.443 & $1029(3)$ & - & This work \\
\hline & & & & 4905.908 & $\mathrm{f}$ & 12 & $5 / 2$ & 9745.334 & $626(1)$ & $5(3)$ & [4] \\
\hline & & & & 5081.628 & $\mathrm{f}$ & 15 & $7 / 2$ & 10449.997 & $541(2)$ & - & [19] \\
\hline & & & & 5107.293 & $\mathrm{nl}, \mathrm{f}$ & 4 & $3 / 2$ & 10548.845 & $34(5)$ & & [19] \\
\hline & & & & 5811.02 & $\mathrm{nl}, \mathrm{e}$ & 1 & $7 / 2$ & 12919.316 & $180(2)$ & - & [19] \\
\hline & & & & 5920.20 & nl, e & 1 & $7 / 2$ & 13236.606 & $726(1)$ & - & [19] \\
\hline & & & & 6186.947 & $\mathrm{nl}, \mathrm{f}$ & 5 & $5 / 2$ & 13964.645 & $185(2)$ & - & [19] \\
\hline & & & & 6204.063 & $\mathrm{nl}$ & 5 & $7 / 2$ & 14009.225 & $1100(1)$ & & [19] \\
\hline \multirow[t]{6}{*}{$9 / 2$} & 30232.222 & $737(2)$ & & 4840.699 & $\mathrm{f}$ & 80 & $9 / 2$ & 9579.820 & $789(1)$ & - & [19] \\
\hline & & & & 5498.333 & $\mathrm{nl}, \mathrm{f}$ & 1 & $9 / 2$ & 12049.942 & $275(2)$ & - & [19] \\
\hline & & & & 5741.84 & $\mathrm{nl}, \mathrm{e}$ & 1 & $9 / 2$ & 12821.044 & $1127(3)$ & - & [19] \\
\hline & & & & 5760.58 & $\mathrm{nl}, \mathrm{e}$ & 1 & $11 / 2$ & 12877.682 & 1139 (2) & - & [19] \\
\hline & & & & 5774.44 & $\mathrm{nl}, \mathrm{e}$ & 1 & $7 / 2$ & 12919.316 & $180(2)$ & - & [19] \\
\hline & & & & 5925.44 & $\mathrm{nl}, \mathrm{e}$ & 1 & $11 / 2$ & 13360.511 & $151(3)$ & - & [19] \\
\hline \multirow[t]{4}{*}{$9 / 2$} & 30356.677 & $788(2)$ & & 4811.703 & $\mathrm{f}$ & 22 & $9 / 2$ & 9579.820 & $789(1)$ & - & [19] \\
\hline & & & & 5529.822 & & 36 & $7 / 2$ & 12277.935 & $1760(4)$ & - & [19] \\
\hline & & & & 5733.22 & $\mathrm{nl}, \mathrm{e}$ & 1 & $7 / 2$ & 12919.316 & $180(2)$ & - & [19] \\
\hline & & & & 5882.05 & $\mathrm{nl}, \mathrm{e}$ & 1 & $11 / 2$ & 13360.511 & $151(3)$ & - & [19] \\
\hline \multirow[t]{6}{*}{$9 / 2$} & 30447.257 & $695(2)$ & & 4790.816 & $\mathrm{f}$ & 18 & $9 / 2$ & 9579.820 & $789(1)$ & - & [19] \\
\hline & & & & 4999.286 & $\mathrm{e}$ & 5 & $7 / 2$ & 10449.997 & $541(2)$ & - & [19] \\
\hline & & & & 5703.59 & $\mathrm{nl}, \mathrm{e}$ & 1 & $7 / 2$ & 12919.316 & $180(2)$ & - & [19] \\
\hline & & & & 5808.79 & $\mathrm{nl}, \mathrm{e}$ & 1 & $7 / 2$ & 13236.606 & $726(1)$ & - & [19] \\
\hline & & & & 5857.48 & $\mathrm{nl}, \mathrm{e}$ & 1 & $9 / 2$ & 13379.788 & $932(3)$ & $10(20)$ & [19] \\
\hline & & & & 6081.770 & $\mathrm{nl}, \mathrm{f}$ & 1 & $7 / 2$ & 14009.225 & $1100(1)$ & - & [19] \\
\hline \multirow[t]{3}{*}{$7 / 2$} & 30461.496 & $692(3)$ & & 4787.551 & $\mathrm{f}$ & 5 & $9 / 2$ & 9579.820 & $789(1)$ & - & [19] \\
\hline & & & & 5667.216 & $\mathrm{nl}, \mathrm{e}$ & 12 & $9 / 2$ & 12821.044 & $1127(3)$ & - & [19] \\
\hline & & & & 5843.786 & $\mathrm{nl}, \mathrm{e}$ & 15 & $9 / 2$ & 13354.043 & $1272(3)$ & - & [19] \\
\hline \multirow[t]{6}{*}{$19 / 2$} & 30466.597 & $403(3)$ & & 5705.81 & $\mathrm{nl}, \mathrm{e}$ & 1 & $19 / 2$ & 12945.474 & $837.1(3)$ & $-7(6)$ & [9] \\
\hline & & & & 5789.467 & $\mathrm{e}$ & 27 & $21 / 2$ & 13198.637 & $785.8(5)$ & $-85(20)$ & {$[9]$} \\
\hline & & & & 5817.01 & $\mathrm{nl}, \mathrm{e}$ & 1 & $17 / 2$ & 13280.404 & $208(2)$ & $20(50)$ & [19] \\
\hline & & & & 6199.288 & $\mathrm{f}$ & 58 & $17 / 2$ & 14340.174 & $245(1)$ & $-20(15)$ & [19] \\
\hline & & & & 6381.18 & $\mathrm{nl}, \mathrm{e}$ & 4 & $19 / 2$ & 14799.842 & $126(5)$ & - & [19] \\
\hline & & & & 8541.018 & & 9 & $19 / 2$ & 18761.608 & $870(1)$ & - & This work \\
\hline \multirow[t]{4}{*}{$5 / 2$} & 30590.721 & $622(2)$ & & 4988.161 & $\mathrm{f}$ & 18 & $3 / 2$ & 10548.845 & $34(5)$ & & [19] \\
\hline & & & & 5760.72 & $\mathrm{nl}, \mathrm{e}$ & 1 & $7 / 2$ & 13236.606 & $726(1)$ & - & [19] \\
\hline & & & & 5767.20 & $\mathrm{nl}, \mathrm{e}$ & 1 & $5 / 2$ & 13256.082 & $1074(3)$ & - & [19] \\
\hline & & & & 5891.36 & $\mathrm{nl}, \mathrm{e}$ & 1 & $7 / 2$ & 13621.400 & $879(1)$ & $-10(10)$ & This work \\
\hline \multirow[t]{7}{*}{$13 / 2$} & 30600.763 & $656(2)$ & & 3420.845 & $\mathrm{nl}$ & 3 & $11 / 2$ & 1376.602 & 730.393 & -11.877 & [7] \\
\hline & & & & 3602.055 & $\mathrm{nl}$ & 4 & $13 / 2$ & 2846.741 & 613.240 & -12.850 & [7] \\
\hline & & & & 5439.581 & $\mathrm{nl}, \mathrm{f}$ & 14 & $13 / 2$ & 12222.091 & $1133(4)$ & - & This work \\
\hline & & & & 5640.794 & $\mathrm{nl}, \mathrm{f}$ & 1 & $11 / 2$ & 12877.682 & $1139(2)$ & - & [19] \\
\hline & & & & 5868.55 & $\mathrm{nl}, \mathrm{e}$ & 1 & $15 / 2$ & 13565.490 & $917(1)$ & $10(10)$ & [19] \\
\hline & & & & 6314.88 & $\mathrm{nl}, \mathrm{e}$ & 1 & $15 / 2$ & 14769.529 & $806(2)$ & - & This work \\
\hline & & & & 6554.134 & & 9 & $13 / 2$ & 15347.431 & $674(2)$ & - & This work \\
\hline
\end{tabular}


Table 4: Continued.

\begin{tabular}{|c|c|c|c|c|c|c|c|c|c|c|c|}
\hline \multicolumn{4}{|c|}{ New upper even level } & \multicolumn{3}{|c|}{ Line } & \multicolumn{5}{|c|}{ Combining known lower odd level } \\
\hline$J$ & Energy $/ \mathrm{cm}^{-1}$ & $\mathrm{~A} / \mathrm{MHz}$ & $\mathrm{B} / \mathrm{MHz}$ & $\lambda / \AA$ & C. & SNR & J & Energy $/ \mathrm{cm}^{-1}$ & $\mathrm{~A} / \mathrm{MHz}$ & $\mathrm{B} / \mathrm{MHz}$ & Reference to col. 10, 11 \\
\hline \multirow{10}{*}{$9 / 2$} & 30625.599 & $643(1)$ & $25(50)$ & 3264.301 & $\mathrm{nl}, \mathrm{f}$ & 15 & $9 / 2$ & 0.000 & 926.209 & -11.878 & {$[7]$} \\
\hline & & & & 4750.218 & $\mathrm{nl}, \mathrm{f}$ & 2 & $9 / 2$ & 9579.820 & $789(1)$ & - & [19] \\
\hline & & & & 4950.613 & $\mathrm{nl}, \mathrm{f}$ & 5 & $11 / 2$ & 10431.716 & $701.7(5)$ & $-10(7)$ & [9] \\
\hline & & & & 5632.903 & $\mathrm{nl}$ & 10 & $11 / 2$ & 12877.682 & $1139(2)$ & - & [19] \\
\hline & & & & 5646.143 & $\mathrm{nl}$ & 7 & $7 / 2$ & 12919.316 & $180(2)$ & - & [19] \\
\hline & & & & 5749.176 & $\mathrm{nl}, \mathrm{e}$ & 6 & $7 / 2$ & 13236.606 & $726(1)$ & - & [19] \\
\hline & & & & 5788.26 & $\mathrm{nl}, \mathrm{e}$ & 1 & $9 / 2$ & 13354.043 & $1272(3)$ & - & [19] \\
\hline & & & & 5790.43 & $\mathrm{nl}$, e & 1 & $11 / 2$ & 13360.511 & $151(3)$ & - & [19] \\
\hline & & & & 5796.90 & $\mathrm{nl}, \mathrm{e}$ & 1 & $9 / 2$ & 13379.788 & $932(3)$ & $10(20)$ & [19] \\
\hline & & & & 6325.752 & & 15 & $11 / 2$ & 14821.565 & $544(2)$ & & This work \\
\hline \multirow[t]{9}{*}{$17 / 2$} & 30826.745 & $580(2)$ & & 5244.434 & $\mathrm{nl}, \mathrm{f}$ & 1 & $17 / 2$ & 11764.216 & $892.5(7)$ & $-10(25)$ & [9] \\
\hline & & & & 5590.891 & $\mathrm{nl}, \mathrm{f}$ & 1 & $19 / 2$ & 12945.474 & $837.1(3)$ & $-7(6)$ & [9] \\
\hline & & & & 5608.63 & $\mathrm{nl}, \mathrm{e}$ & 1 & $15 / 2$ & 13002.023 & $317(2)$ & $30(50)$ & [19] \\
\hline & & & & 5791.715 & $\mathrm{e}$ & 45 & $15 / 2$ & 13565.490 & $917(1)$ & $10(10)$ & [19] \\
\hline & & & & 6063.863 & $\mathrm{nl}, \mathrm{f}$ & 1 & $17 / 2$ & 14340.174 & $245(1)$ & $-20(15)$ & {$[19]$} \\
\hline & & & & 6226.007 & $\mathrm{nl}, \mathrm{f}$ & 1 & $15 / 2$ & 14769.529 & $806(2)$ & - & This work \\
\hline & & & & 6294.331 & $\mathrm{nl}$ & 1 & $17 / 2$ & 14943.825 & $808(3)$ & - & [19] \\
\hline & & & & 6394.270 & $\mathrm{nl}, \mathrm{e}$ & 9 & $15 / 2$ & 15192.075 & $730(5)$ & & This work \\
\hline & & & & 7160.474 & $\mathrm{nl}$ & 4 & $15 / 2$ & 16865.034 & $291(4)$ & & This work \\
\hline \multirow[t]{7}{*}{$17 / 2$} & 30848.885 & $507(1)$ & & 5238.350 & $\mathrm{nl}, \mathrm{f}$ & 1 & $17 / 2$ & 11764.216 & 892.5 (7) & $-10(25)$ & [9] \\
\hline & & & & 5583.974 & $\mathrm{f}$ & 14 & $19 / 2$ & 12945.474 & $837.1(3)$ & $-7(6)$ & {$[9]$} \\
\hline & & & & 5690.43 & $\mathrm{nl}, \mathrm{e}$ & 1 & $17 / 2$ & 13280.404 & $208(2)$ & $20(50)$ & [19] \\
\hline & & & & 5784.296 & $\mathrm{e}$ & 15 & $15 / 2$ & 13565.490 & $917(1)$ & $10(10)$ & [19] \\
\hline & & & & 6055.730 & $\mathrm{nl}$ & 7 & $17 / 2$ & 14340.174 & $245(1)$ & $-20(15)$ & [19] \\
\hline & & & & 6217.433 & & 8 & $15 / 2$ & 14769.529 & $806(2)$ & - & This work \\
\hline & & & & 6385.226 & $\mathrm{nl}, \mathrm{e}$ & 12 & $15 / 2$ & 15192.075 & $730(5)$ & & This work \\
\hline \multirow[t]{5}{*}{$5 / 2$} & 30963.451 & $661(2)$ & & 4873.488 & $\mathrm{f}$ & 30 & $7 / 2$ & 10449.997 & $541(2)$ & - & [19] \\
\hline & & & & 4897.088 & $\mathrm{nl}$ & 3 & $3 / 2$ & 10548.845 & $34(5)$ & & [19] \\
\hline & & & & 4922.460 & $\mathrm{nl}$ & 3 & $7 / 2$ & 10654.070 & $169(2)$ & - & This work \\
\hline & & & & 5764.73 & $\mathrm{nl}$, e & 1 & $7 / 2$ & 13621.400 & $879(1)$ & $-10(10)$ & This work \\
\hline & & & & 5881.14 & $\mathrm{nl}, \mathrm{e}$ & 1 & $5 / 2$ & 13964.645 & $185(2)$ & - & [19] \\
\hline \multirow[t]{5}{*}{$7 / 2$} & 31005.715 & $692(2)$ & & 4665.944 & $\mathrm{f}$ & 5 & $9 / 2$ & 9579.820 & $789(1)$ & - & {$[19]$} \\
\hline & & & & 5080.914 & $\mathrm{f}$ & 12 & $9 / 2$ & 11329.696 & $530(3)$ & - & [19] \\
\hline & & & & 5273.970 & $\mathrm{nl}, \mathrm{f}$ & 1 & $9 / 2$ & 12049.942 & $275(2)$ & - & [19] \\
\hline & & & & 5750.72 & $\mathrm{nl}, \mathrm{e}$ & 1 & $7 / 2$ & 13621.400 & $879(1)$ & $-10(10)$ & This work \\
\hline & & & & 5866.55 & $\mathrm{nl}, \mathrm{e}$ & 1 & $5 / 2$ & 13964.645 & $185(2)$ & - & [19] \\
\hline \multirow[t]{4}{*}{$11 / 2$} & 31010.172 & $705(2)$ & & 5079.762 & $\mathrm{nl}, \mathrm{f}$ & 3 & $9 / 2$ & 11329.696 & $530(3)$ & - & {$[19]$} \\
\hline & & & & 5316.005 & $\mathrm{nl}$ & 5 & $11 / 2$ & 12204.286 & $1010(2)$ & - & This work \\
\hline & & & & 5875.80 & $\mathrm{nl}, \mathrm{e}$ & 1 & $13 / 2$ & 13995.931 & $1067(1)$ & $200(50)$ & [19] \\
\hline & & & & 5878.00 & $\mathrm{nl}, \mathrm{e}$ & 1 & $11 / 2$ & 14002.294 & $566(1)$ & $-125 \mathrm{v}$ & {$[19]$} \\
\hline \multirow[t]{3}{*}{$5 / 2$} & 31299.695 & $800(3)$ & & 4794.892 & $\mathrm{f}$ & 25 & $7 / 2$ & 10449.997 & $541(2)$ & - & [19] \\
\hline & & & & 5767.06 & $\mathrm{nl}, \mathrm{e}$ & 1 & $5 / 2$ & 13964.645 & $185(2)$ & - & [19] \\
\hline & & & & 5781.93 & $\mathrm{nl}, \mathrm{e}$ & 1 & $7 / 2$ & 14009.225 & $1100(1)$ & - & [19] \\
\hline \multirow[t]{5}{*}{$15 / 2$} & 31402.846 & $631(2)$ & & 5743.251 & $\mathrm{nl}$ & 3 & $13 / 2$ & 13995.931 & $1067(1)$ & $200(50)$ & [19] \\
\hline & & & & 5918.099 & $\mathrm{e}$ & 10 & $13 / 2$ & 14510.207 & $1085(2)$ & - & This work \\
\hline & & & & 6166.705 & $\mathrm{nl}, \mathrm{e}$ & 30 & $13 / 2$ & 15191.218 & $666(5)$ & & This work \\
\hline & & & & 6166.957 & $\mathrm{f}, \mathrm{e}$ & 50 & $13 / 2$ & 15191.891 & $730(5)$ & & This work \\
\hline & & & & 6167.03 & $\mathrm{nl}, \mathrm{e}$ & 1 & $15 / 2$ & 15192.075 & $730(5)$ & & This work \\
\hline
\end{tabular}


Table 4: Continued.

\begin{tabular}{|c|c|c|c|c|c|c|c|c|c|c|c|}
\hline \multicolumn{4}{|c|}{ New upper even level } & \multicolumn{3}{|c|}{ Line } & \multicolumn{5}{|c|}{ Combining known lower odd level } \\
\hline$J$ & Energy/ $\mathrm{cm}^{-1}$ & $\mathrm{~A} / \mathrm{MHz}$ & $\mathrm{B} / \mathrm{MHz}$ & $\lambda / \AA$ & C. & SNR & $J$ & Energy $/ \mathrm{cm}^{-1}$ & $\mathrm{~A} / \mathrm{MHz}$ & $\mathrm{B} / \mathrm{MHz}$ & Reference to col. 10,11 \\
\hline \multirow[t]{8}{*}{$11 / 2$} & 31664.262 & $601(2)$ & & 4916.362 & $\mathrm{f}$ & 12 & $9 / 2$ & 11329.696 & $530(3)$ & - & {$[19]$} \\
\hline & & & & 5658.274 & $\mathrm{nl}$ & 12 & $13 / 2$ & 13995.931 & $1067(1)$ & $200(50)$ & [19] \\
\hline & & & & 5733.00 & $\mathrm{nl}, \mathrm{e}$ & 1 & $11 / 2$ & 14226.220 & $869(3)$ & - & [19] \\
\hline & & & & 5827.91 & nl, e & 1 & $13 / 2$ & 14510.207 & $1085(2)$ & - & This work \\
\hline & & & & 5836.135 & nl, e & 3 & $9 / 2$ & 14534.393 & $100(2)$ & - & [19] \\
\hline & & & & 5903.148 & $\mathrm{nl}, \mathrm{f}$ & 1 & $11 / 2$ & 14728.843 & $811(2)$ & - & [19] \\
\hline & & & & 5935.647 & $\mathrm{nl}, \mathrm{e}$ & 5 & $11 / 2$ & 14821.565 & $544(2)$ & & This work \\
\hline & & & & 6145.287 & $\mathrm{nl}, \mathrm{e}$ & 7 & $9 / 2$ & 15396.135 & $719(8)$ & & This work \\
\hline \multirow[t]{4}{*}{$9 / 2$} & 31677.736 & $433(2)$ & & 4913.107 & $\mathrm{nl}, \mathrm{f}$ & 35 & $9 / 2$ & 11329.696 & $530(3)$ & - & [19] \\
\hline & & & & 5728.571 & nl, e & 6 & $11 / 2$ & 14226.220 & $869(3)$ & - & [19] \\
\hline & & & & 5831.55 & $\mathrm{nl}$, e & 1 & $9 / 2$ & 14534.393 & $100(2)$ & - & [19] \\
\hline & & & & 5898.450 & $\mathrm{nl}$ & 5 & $11 / 2$ & 14728.843 & $811(2)$ & - & [19] \\
\hline \multirow[t]{3}{*}{$11 / 2$} & 31771.680 & $718(2)$ & & 5055.597 & $\mathrm{f}$ & 14 & $11 / 2$ & 11997.137 & $585(3)$ & - & [19] \\
\hline & & & & 5799.77 & $\mathrm{nl}, \mathrm{e}$ & 1 & $9 / 2$ & 14534.393 & $100(2)$ & - & [19] \\
\hline & & & & 5865.94 & $\mathrm{nl}, \mathrm{e}$ & 1 & $11 / 2$ & 14728.843 & $811(2)$ & - & [19] \\
\hline \multirow[t]{3}{*}{$11 / 2$} & 31900.665 & $525(2)$ & & 5385.041 & $\mathrm{nl}, \mathrm{f}$ & 5 & $13 / 2$ & 13335.868 & 895 (1) & $100(50)$ & [19] \\
\hline & & & & 5748.69 & $\mathrm{nl}, \mathrm{e}$ & 1 & $13 / 2$ & 14510.207 & $1085(2)$ & - & This work \\
\hline & & & & 5821.88 & $\mathrm{nl}, \mathrm{e}$ & 1 & $11 / 2$ & 14728.843 & $811(2)$ & - & [19] \\
\hline \multirow[t]{4}{*}{$7 / 2$} & 32070.559 & $668(2)$ & & 4820.053 & $\mathrm{f}$ & 20 & $9 / 2$ & 11329.696 & $530(3)$ & - & [19] \\
\hline & & & & 5700.92 & $\mathrm{nl}, \mathrm{e}$ & 1 & $9 / 2$ & 14534.393 & $100(2)$ & - & [19] \\
\hline & & & & 5881.09 & nl, e & 1 & $9 / 2$ & 15071.618 & $635(3)$ & - & [19] \\
\hline & & & & 5995.55 & $\mathrm{nl}, \mathrm{e}, \mathrm{f}$ & 1 & $9 / 2$ & 15396.135 & $719(8)$ & & This work \\
\hline \multirow[t]{3}{*}{$7 / 2$} & 32166.080 & $736(2)$ & & 4797.956 & $\mathrm{f}$ & 18 & $9 / 2$ & 11329.696 & $530(3)$ & - & [19] \\
\hline & & & & 5670.03 & $\mathrm{nl}, \mathrm{e}$ & 1 & $9 / 2$ & 14534.393 & $100(2)$ & - & [19] \\
\hline & & & & 5695.74 & $\mathrm{nl}, \mathrm{e}$ & 1 & $7 / 2$ & 14613.96 & $760(3)$ & - & [19] \\
\hline \multirow[t]{6}{*}{$15 / 2$} & 32336.849 & 475 (1) & & 5690.809 & $\mathrm{nl}, \mathrm{e}$ & 15 & $15 / 2$ & 14769.529 & $806(2)$ & - & This work \\
\hline & & & & 5747.837 & nl, e & 30 & $17 / 2$ & 14943.825 & 808 (3) & - & [19] \\
\hline & & & & 5772.48 & $\mathrm{nl}, \mathrm{e}$ & 1 & $13 / 2$ & 15018.088 & $108(3)$ & - & [19] \\
\hline & & & & 5884.389 & $\mathrm{f}$ & 18 & $13 / 2$ & 15347.431 & $674(2)$ & - & This work \\
\hline & & & & 5902.322 & nl, e & 6 & $15 / 2$ & 15399.063 & $889(2)$ & - & This work \\
\hline & & & & 5911.88 & nl, e & 1 & $13 / 2$ & 15426.436 & 737 (2) & - & This work \\
\hline \multirow[t]{7}{*}{$11 / 2$} & 32373.816 & $660(2)$ & & 3225.165 & $\mathrm{nl}$ & 12 & $11 / 2$ & 1376.602 & 730.393 & -11.877 & [7] \\
\hline & & & & 4906.194 & $\mathrm{f}$ & 12 & $11 / 2$ & 11997.137 & $585(3)$ & - & [19] \\
\hline & & & & 5695.696 & $\mathrm{nl}$ & 10 & $11 / 2$ & 14821.565 & $544(2)$ & & This work \\
\hline & & & & 5724.69 & $\mathrm{nl}, \mathrm{e}$ & 1 & $9 / 2$ & 14910.476 & $611(2)$ & - & [19] \\
\hline & & & & 5760.19 & $\mathrm{nl}, \mathrm{e}$ & 1 & $13 / 2$ & 15018.088 & $108(3)$ & - & [19] \\
\hline & & & & 5871.61 & nl, e & 1 & $13 / 2$ & 15347.431 & $674(2)$ & - & This work \\
\hline & & & & 5979.354 & $\mathrm{e}$ & 12 & $13 / 2$ & 15654.235 & $577(1)$ & & This work \\
\hline \multirow[t]{6}{*}{$9 / 2$} & 32402.860 & $544(2)$ & & 4553.938 & $\mathrm{nl}, \mathrm{f}$ & 1 & $7 / 2$ & 10449.997 & $541(2)$ & - & [19] \\
\hline & & & & 4744.046 & $\mathrm{nl}, \mathrm{f}$ & 25 & $9 / 2$ & 11329.696 & $530(3)$ & - & [19] \\
\hline & & & & 5656.45 & nl, e & 1 & $11 / 2$ & 14728.843 & $811(2)$ & - & [19] \\
\hline & & & & 5715.19 & $\mathrm{nl}, \mathrm{e}$ & 1 & $9 / 2$ & 14910.476 & $611(2)$ & - & [19] \\
\hline & & & & 5768.327 & $\mathrm{nl}, \mathrm{e}$ & 17 & $9 / 2$ & 15071.618 & $635(3)$ & - & [19] \\
\hline & & & & 5878.396 & $\mathrm{nl}, \mathrm{e}$ & 12 & $9 / 2$ & 15396.135 & $719(8)$ & & This work \\
\hline \multirow[t]{5}{*}{$13 / 2$} & 32623.126 & $591(2)$ & & 3357.401 & $\mathrm{nl}$ & 10 & $13 / 2$ & 2846.741 & 613.240 & -12.850 & {$[7]$} \\
\hline & & & & 5434.186 & $\mathrm{nl}, \mathrm{f}$ & 1 & $11 / 2$ & 14226.220 & 869 (3) & - & [19] \\
\hline & & & & 5678.62 & $\mathrm{nl}, \mathrm{e}$ & 1 & $13 / 2$ & 15018.088 & $108(3)$ & - & [19] \\
\hline & & & & 5786.874 & nl, e & 5 & $13 / 2$ & 15347.431 & $674(2)$ & - & This work \\
\hline & & & & 5891.506 & $\mathrm{e}$ & 14 & $13 / 2$ & 15654.235 & $577(1)$ & & This work \\
\hline
\end{tabular}


TABle 4: Continued.

\begin{tabular}{|c|c|c|c|c|c|c|c|c|c|c|c|}
\hline \multirow[b]{2}{*}{$J$} & \multicolumn{3}{|c|}{ New upper even level } & \multicolumn{3}{|c|}{ Line } & \multicolumn{5}{|c|}{ Combining known lower odd level } \\
\hline & Energy $/ \mathrm{cm}^{-1}$ & $\mathrm{~A} / \mathrm{MHz}$ & $\mathrm{B} / \mathrm{MHz}$ & $\lambda / \AA$ & C. & SNR & J & Energy/ $/ \mathrm{cm}^{-1}$ & $\mathrm{~A} / \mathrm{MHz}$ & $\mathrm{B} / \mathrm{MHz}$ & Reference to col. 10, 11 \\
\hline \multirow[t]{11}{*}{$15 / 2$} & 33098.154 & $480(2)$ & & 3481.252 & $\mathrm{nl}$, & 2 & $15 / 2$ & 4381.072 & 541.575 & -14.558 & {$[7]$} \\
\hline & & & & 5454.430 & $\mathrm{nl}, \mathrm{f}$ & 6 & $15 / 2$ & 14769.529 & $806(2)$ & - & This work \\
\hline & & & & 5506.798 & $\mathrm{f}$ & 17 & $17 / 2$ & 14943.825 & $808(3)$ & - & [19] \\
\hline & & & & 5529.417 & $\mathrm{nl}, \mathrm{f}$ & 1 & $13 / 2$ & 15018.088 & $108(3)$ & - & [19] \\
\hline & & & & 5582.878 & $\mathrm{nl}, \mathrm{f}$ & 1 & $13 / 2$ & 15191.218 & $666(5)$ & & This work \\
\hline & & & & 5632.008 & $\mathrm{e}$ & 10 & $13 / 2$ & 15347.431 & $674(2)$ & - & This work \\
\hline & & & & 5648.443 & $\mathrm{nl}, \mathrm{e}$ & $1^{*}$ & $15 / 2$ & 15399.063 & $887(3)$ & - & This work \\
\hline & & & & 5657.192 & $\mathrm{nl}, \mathrm{e}$ & $1^{*}$ & $13 / 2$ & 15426.436 & $737(2)$ & - & This work \\
\hline & & & & 5917.931 & $\mathrm{e}$ & 10 & $13 / 2$ & 16205.041 & $733(2)$ & - & This work \\
\hline & & & & 6128.250 & $\mathrm{nl}, \mathrm{f}$ & 1 & $15 / 2$ & 16784.797 & $297(5)$ & - & This work \\
\hline & & & & 6520.162 & $\mathrm{nl}, \mathrm{f}$ & 1 & $13 / 2$ & 17765.348 & $490(3)$ & - & {$[19]$} \\
\hline
\end{tabular}

C: comment; nl: new line; e: excited; f: observed as fluorescence line. Lines, for which SNR 1 is given, do not show up in our FT spectrum, but could be excited or were observed as fluorescence lines.

transitions between already known levels (in the wavelength range 3977 to $9878 \AA$ ) and to the discovery of 23 new levels [11]. But it turned out that this spectrum is also very helpful in improving earlier laser spectroscopic data, since a huge number of excited lines and/or lines observed as fluorescence transitions can be clearly identified in the FT spectrum due to their characteristic hf patterns. Moreover, after calculating all possible combinations from a level together with their expected hf patterns, one can have a look at the FT spectrum if these patterns appear and classify in this way spectral lines. Having determined the A-factors from the laser spectroscopic recording, the cg wavelength can be determined with an accuracy of $0.001 \AA$ adjusting a calculated hf pattern to the pattern of the line taken from the FT spectrum. In this way, the cg wave number of the line can be determined with high accuracy. Using already corrected energy values of known levels, the energy of the new levels could be determined now with an accuracy of $0.010 \mathrm{~cm}^{-1}$.

Not all of the excited transitions appear clearly in the FT spectrum. Due to the huge number of energy levels, blend situations, in which different pairs of combining levels possess the same wave number difference, are observed quite frequently. Then the corresponding hf patterns overlap, so one observes their sum curve, weighted with their relative intensities, in the FT spectrum. In such cases, it is not always possible to determine reliable cg wavelengths. It is also possible, that a transition is quite weak in the emission spectrum, thus the corresponding line has an intensity below the noise of the FT spectrum. Nevertheless, sometimes it is possible to excite such a weak transition and to observe a LIF signal with good signal-to-noise ratio (SNR). Sometimes also LIF signals can be observed having wavelengths not appearing in our FT spectrum. Most of the fluorescence decays of the new levels were clearly visible lines in the FT spectrum with high SNR. In these cases, their cg wave number could be used to determine the energy of the decaying level with good accuracy.

Only for a few new levels neither the excited nor the fluorescence lines showed up in the FT spectrum, and it was also not possible to find other lines that could be explained as transitions combining with the new level. In such cases, we used a new method to determine the excitation wavelength with high accuracy (explanation see Example 2).

\section{Examples for Finding New Levels}

Example 1. Level pair $12646.996(10) \mathrm{cm}^{-1}, J=5 / 2$, even parity, $A=892(2) \mathrm{MHz}-30304.145(10) \mathrm{cm}^{-1}, J=5 / 2$, odd parity, $A=818(2) \mathrm{MHz}$.

When exciting in the hollow cathode plasma with an estimated cg wavelength $\lambda=5746.49 \AA$, we observed an hf pattern, which could be attributed to a transition between two levels having both $J=5 / 2, A_{\text {up }}=818 \mathrm{MHz}$, and $A_{\mathrm{lo}}=892 \mathrm{MHz}$. Fluorescence was observed on several lines, amongst them $\lambda_{\mathrm{fl}}=5919 \AA$ and $4485 \AA$ (the reading of the monochromator is accurate to $\pm 2 \AA$ ). None of the levels in our database possessed matching $J$ - and $A$-values, thus we had to assume that for this transition two unknown levels combine. Fortunately, one of the fluorescence lines, $5919 \AA$, was in the range of our dye laser. Thus we tuned the monochromator, used for selecting the LIF wavelength, to the second fluorescence line $\lambda_{\mathrm{fl}}=4485 \AA$. The laser wavelength was then set to $\lambda_{\text {start }}=5919 \AA$ and tuned until an LIF signal was observed (at $\lambda=5919.58 \AA$ ). Again the observed hf pattern was evaluated, and this time the lower level could be identified to be $13415.739 \mathrm{~cm}^{-1}$, even parity, $J=7 / 2, A=797 \mathrm{MHz}$, found by us earlier. This allowed us to calculate the energies of the combining levels of the line $\lambda=5746.49 \AA$. Later, with the help of the wave numbers and hf patterns of the fluorescence lines, we could determine the energies with high accuracy.

Example 2. Level $28889.260(10) \mathrm{cm}^{-1}, J=11 / 2$, even parity, $A=639(1) \mathrm{MHz}$.

This level was discovered when tuning the exciting laser wavelength to $\lambda=5826.37 \AA$ (a small peak in the FT spectrum). A strong LIF signal was detected at wavelength $\lambda_{\mathrm{fl}}=5177 \AA$. The recorded hf pattern could be interpreted 
TABLE 5: Spectral lines explained by the new odd levels.

\begin{tabular}{|c|c|c|c|c|c|c|c|c|c|c|c|}
\hline \multicolumn{4}{|c|}{ New upper odd level } & \multicolumn{3}{|c|}{ Line } & \multicolumn{5}{|c|}{ Combining known lower even level } \\
\hline$J$ & Energy/ $\mathrm{cm}^{-1}$ & $\mathrm{~A} / \mathrm{MHz}$ & $\mathrm{B} / \mathrm{MHz}$ & $\lambda / \AA$ & C. & SNR & $J$ & Energy $/ \mathrm{cm}^{-1}$ & $\mathrm{~A} / \mathrm{MHz}$ & $\mathrm{B} / \mathrm{MHz}$ & Reference to col. 10, 11 \\
\hline \multirow[t]{6}{*}{$13 / 2$} & 24781.116 & $858(2)$ & $-20(10)$ & 5020.041 & $\mathrm{nl}$ & 14 & $11 / 2$ & 4866.515 & 867.997 & -50.319 & {$[7]$} \\
\hline & & & & 5588.731 & $\mathrm{f}$ & 60 & $11 / 2$ & 6892.934 & 551.934 & -24.736 & {$[7]$} \\
\hline & & & & 5828.953 & e & 70 & $13 / 2$ & 7630.132 & 776.286 & -43.592 & {$[7]$} \\
\hline & & & & 5940.199 & $\mathrm{nl}, \mathrm{e}$ & 75 & $13 / 2$ & 7951.323 & $644(1)$ & $-30(20)$ & [19] \\
\hline & & & & 6535.168 & & 15 & $11 / 2$ & 9483.518 & $731(1)$ & $-15(10)$ & [19] \\
\hline & & & & 6985.811 & $\mathrm{nl}$ & 6 & $13 / 2$ & 10470.329 & $628(3)$ & - & [19] \\
\hline \multirow[t]{5}{*}{$7 / 2$} & 24899.621 & $961(2)$ & & 4884.455 & $\mathrm{f}$ & 170 & $9 / 2$ & 4432.225 & $923.2(4)$ & $-22(7)$ & {$[8]$} \\
\hline & & & & 5240.529 & & 35 & $9 / 2$ & 5822.890 & $855.8(4)$ & $-17(7)$ & {$[8]$} \\
\hline & & & & 5920.24 & nl, e & 5 & $7 / 2$ & 8013.089 & $168(1)$ & - & This work \\
\hline & & & & 6581.885 & & 7 & $5 / 2$ & 9710.600 & $164(2)$ & - & [19] \\
\hline & & & & 6673.086 & & 20 & $7 / 2$ & 9918.190 & $1057.4(5)$ & $22(6)$ & {$[8]$} \\
\hline \multirow[t]{5}{*}{$7 / 2$} & 25172.325 & $708(2)$ & & 5340.248 & $\mathrm{nl}$ & 30 & $5 / 2$ & 6451.808 & $1189(6)$ & $-5(5)$ & {$[8]$} \\
\hline & & & & 5364.250 & $\mathrm{f}$ & 80 & $7 / 2$ & 6535.572 & 979 (1) & $25(30)$ & [19] \\
\hline & & & & 5826.151 & $\mathrm{nl}, \mathrm{e}$ & 5 & $7 / 2$ & 8013.089 & $168(1)$ & - & This work \\
\hline & & & & 5831.653 & $\mathrm{nl}, \mathrm{e}$ & 26 & $9 / 2$ & 8029.275 & 797 (2) & - & [19] \\
\hline & & & & 7338.656 & $\mathrm{nl}$ & 6 & $9 / 2$ & 11549.602 & $1064(2)$ & - & This work \\
\hline \multirow[t]{7}{*}{$9 / 2$} & 25634.179 & $674(1)$ & & 4715.227 & $\mathrm{f}$ & 30 & $9 / 2$ & 4432.225 & $923.2(4)$ & $-22(7)$ & {$[8]$} \\
\hline & & & & 4813.833 & & 8 & $11 / 2$ & 4866.515 & 867.997 & -50.319 & [7] \\
\hline & & & & 5673.44 & $\mathrm{nl}, \mathrm{e}$ & 1 & $7 / 2$ & 8013.089 & $168(1)$ & - & This work \\
\hline & & & & 5774.092 & $\mathrm{nl}, \mathrm{e}$ & 9 & $9 / 2$ & 8320.240 & $255(2)$ & - & [19] \\
\hline & & & & 6048.242 & & 18 & $9 / 2$ & 9105.021 & $689.7(3)$ & $-3(5)$ & {$[8]$} \\
\hline & & & & 6108.743 & & 18 & $11 / 2$ & 9268.726 & 977 (1) & $-24(20)$ & [19] \\
\hline & & & & 6275.949 & & 20 & $7 / 2$ & 9704.744 & $779(1)$ & $-50(30)$ & [19] \\
\hline \multirow[t]{9}{*}{$11 / 2$} & 25900.352 & $858(2)$ & & 4656.763 & $\mathrm{f}$ & 60 & $9 / 2$ & 4432.225 & $923.2(4)$ & $-22(7)$ & {$[8]$} \\
\hline & & & & 4752.913 & $\mathrm{nl}$ & 6 & $11 / 2$ & 4866.515 & 867.997 & -50.319 & [7] \\
\hline & & & & 5180.774 & & 55 & $13 / 2$ & 6603.591 & 755.456 & -48.633 & {$[7]$} \\
\hline & & & & 5569.785 & $\mathrm{nl}$ & 20 & $13 / 2$ & 7951.323 & $644(1)$ & $-30(20)$ & [19] \\
\hline & & & & 5686.67 & $\mathrm{nl}, \mathrm{e}$ & 1 & $9 / 2$ & 8320.240 & $255(2)$ & - & [19] \\
\hline & & & & 5793.302 & $\mathrm{e}$ & 60 & $9 / 2$ & 8643.824 & $797(2)$ & - & [19] \\
\hline & & & & 5856.16 & $\mathrm{nl}, \mathrm{e}$ & 1 & $11 / 2$ & 8829.063 & $769(1)$ & $-30(20)$ & This work \\
\hline & & & & 6161.500 & & 15 & $11 / 2$ & 9675.029 & $683(1)$ & - & [19] \\
\hline & & & & 9469.298 & & 15 & $9 / 2$ & 15342.804 & $1103(5)$ & - & This work \\
\hline \multirow[t]{4}{*}{$17 / 2$} & 25909.173 & $854(2)$ & $-45(5)$ & 5697.960 & $e, f$ & 120 & $15 / 2$ & 8363.901 & 763.306 & -48.253 & [7] \\
\hline & & & & 5831.453 & $e, f$ & 210 & $15 / 2$ & 8765.542 & 763.557 & -45.805 & {$[7]$} \\
\hline & & & & 7589.454 & & 13 & $17 / 2$ & 12736.621 & 904 (1) & $20(20)$ & This work \\
\hline & & & & 8139.425 & $\mathrm{nl}$ & 5 & $19 / 2$ & 13626.672 & $865(3)$ & - & [19] \\
\hline \multirow[t]{11}{*}{$11 / 2$} & 26159.533 & $672(3)$ & & 4601.217 & $\mathrm{nl}$ & 4 & $9 / 2$ & 4432.225 & $923.2(4)$ & $-22(7)$ & {$[8]$} \\
\hline & & & & 4915.860 & $\mathrm{f}$ & 40 & $9 / 2$ & 5822.890 & $855.8(4)$ & $-17(7)$ & {$[8]$} \\
\hline & & & & 5037.316 & $\mathrm{nl}$ & 46 & $11 / 2$ & 6313.224 & $756(1)$ & - & This work \\
\hline & & & & 5112.113 & $\mathrm{nl}$ & 29 & $13 / 2$ & 6603.591 & 755.456 & -48.633 & [7] \\
\hline & & & & 5188.885 & $\mathrm{nl}$ & 11 & $11 / 2$ & 6892.934 & 551.934 & -24.736 & {$[7]$} \\
\hline & & & & 5514.111 & & 75 & $9 / 2$ & 8029.275 & 797 (2) & - & [19] \\
\hline & & & & 5707.577 & $\mathrm{nl}, \mathrm{e}$ & 10 & $9 / 2$ & 8643.824 & $797(2)$ & - & [19] \\
\hline & & & & 5861.933 & $\mathrm{nl}, \mathrm{e}$ & 7 & $9 / 2$ & 9105.021 & $689.7(3)$ & $-3(5)$ & {$[8]$} \\
\hline & & & & 6720.082 & & 8 & $11 / 2$ & 11282.865 & $1049(2)$ & - & This work \\
\hline & & & & 6848.946 & $\mathrm{nl}$ & 5 & $13 / 2$ & 11562.762 & $819(2)$ & - & This work \\
\hline & & & & 9697.559 & $\mathrm{nl}$ & 5 & $13 / 2$ & 15850.483 & $926(3)$ & - & [19] \\
\hline \multirow[t]{3}{*}{$15 / 2$} & 26332.040 & $506(2)$ & $-45(5)$ & 5067.409 & & 85 & $13 / 2$ & 6603.591 & 755.456 & -48.633 & [7] \\
\hline & & & & 5345.561 & $\mathrm{f}$ & 62 & $13 / 2$ & 7630.132 & 776.286 & -43.592 & [7] \\
\hline & & & & 5691.075 & $\mathrm{e}$ & 36 & $15 / 2$ & 8765.542 & 763.557 & -45.805 & [7] \\
\hline
\end{tabular}


Table 5: Continued.

\begin{tabular}{|c|c|c|c|c|c|c|c|c|c|c|c|}
\hline \multicolumn{4}{|c|}{ New upper odd level } & \multicolumn{3}{|c|}{ Line } & \multicolumn{5}{|c|}{ Combining known lower even level } \\
\hline \multirow[t]{4}{*}{$J$} & Energy $/ \mathrm{cm}^{-1}$ & $\mathrm{~A} / \mathrm{MHz}$ & $\mathrm{B} / \mathrm{MHz}$ & $\lambda / \AA$ & C. & SNR & $J$ & Energy $/ \mathrm{cm}^{-1}$ & $\mathrm{~A} / \mathrm{MHz}$ & $\mathrm{B} / \mathrm{MHz}$ & Reference to col. 10, 11 \\
\hline & & & & 5926.883 & $\mathrm{e}$ & 110 & $13 / 2$ & 9464.440 & $1056(1)$ & $-15(10)$ & {$[19]$} \\
\hline & & & & 6222.781 & & 9 & $13 / 2$ & 10266.501 & $972(2)$ & - & This work \\
\hline & & & & 7390.274 & $\mathrm{nl}$ & 6 & $15 / 2$ & 12804.468 & $732(1)$ & 一 & This work \\
\hline \multirow[t]{13}{*}{$9 / 2$} & 26337.481 & $810(1)$ & & 4563.835 & $\mathrm{f}$ & 30 & $9 / 2$ & 4432.225 & $923.2(4)$ & $-22(7)$ & [8] \\
\hline & & & & 4656.148 & $\mathrm{f}$ & 8 & $11 / 2$ & 4866.515 & 867.997 & -50.319 & [7] \\
\hline & & & & 4873.218 & $\mathrm{f}$ & 20 & $9 / 2$ & 5822.890 & $855.8(4)$ & $-17(7)$ & {$[8]$} \\
\hline & & & & 4992.551 & & 8 & $11 / 2$ & 6313.224 & $756(1)$ & - & This work \\
\hline & & & & 5141.397 & $\mathrm{nl}$ & 14 & $11 / 2$ & 6892.934 & 551.934 & -24.736 & [7] \\
\hline & & & & 5340.384 & $\mathrm{nl}$ & 8 & $7 / 2$ & 7617.440 & 866.9 (5) & $-4(5)$ & {$[8]$} \\
\hline & & & & 5801.393 & $\mathrm{nl}$ & 7 & $9 / 2$ & 9105.021 & $689.7(3)$ & $-3(5)$ & {$[8]$} \\
\hline & & & & 5931.678 & & 13 & $11 / 2$ & 9483.518 & $731(1)$ & $-15(10)$ & [19] \\
\hline & & & & 7736.76 & nl, e & 1 & $7 / 2$ & 13415.739 & $797(2)$ & - & This work \\
\hline & & & & 7842.72 & $\mathrm{nl}$, e & 1 & $7 / 2$ & 13590.311 & $1151(2)$ & - & [19] \\
\hline & & & & 7852.18 & nl, e & 1 & $9 / 2$ & 13605.665 & $681(2)$ & - & This work \\
\hline & & & & 7928.03 & $\mathrm{nl}, \mathrm{e}$ & 1 & $11 / 2$ & 13727.482 & 949 (1) & $-30(20)$ & [19] \\
\hline & & & & 7935.73 & $\mathrm{nl}, \mathrm{e}$ & 1 & $7 / 2$ & 13739.706 & $1128(1)$ & - & [19] \\
\hline \multirow[t]{8}{*}{$7 / 2$} & 26371.971 & $997(3)$ & & 4556.661 & $\mathrm{nl}$ & 6 & $9 / 2$ & 4432.225 & $923.2(4)$ & $-22(7)$ & {$[8]$} \\
\hline & & & & 5039.832 & $\mathrm{f}$ & 45 & $7 / 2$ & 6535.572 & 979 (1) & $25(30)$ & [19] \\
\hline & & & & 5330.562 & $\mathrm{f}$ & 40 & $7 / 2$ & 7617.440 & 866.9 (5) & $-4(5)$ & {$[8]$} \\
\hline & & & & 5445.441 & $\mathrm{nl}, \mathrm{f}$ & 1 & $7 / 2$ & 8013.089 & $168(1)$ & - & This work \\
\hline & & & & 6242.330 & $\mathrm{nl}$ & 12 & $9 / 2$ & 10356.737 & $1406(1)$ & - & This work \\
\hline & & & & 6549.43 & nl, e & 1 & $5 / 2$ & 11107.696 & $658(2)$ & - & This work \\
\hline & & & & 6582.51 & $\mathrm{nl}, \mathrm{e}$ & 1 & $9 / 2$ & 11184.396 & $692(1)$ & $15(30)$ & This work \\
\hline & & & & 7217.048 & $\mathrm{nl}$ & 10 & $9 / 2$ & 12519.705 & $693(3)$ & - & [19] \\
\hline \multirow[t]{10}{*}{$9 / 2$} & 26718.738 & $826(2)$ & & 4574.911 & $\mathrm{nl}$ & 16 & $11 / 2$ & 4866.515 & 867.997 & -50.319 & {$[7]$} \\
\hline & & & & 4784.302 & & 25 & $9 / 2$ & 5822.890 & $855.8(4)$ & $-17(7)$ & {$[8]$} \\
\hline & & & & 4899.268 & & 20 & $11 / 2$ & 6313.224 & $756(1)$ & - & This work \\
\hline & & & & 4997.468 & $\mathrm{f}$ & 15 & $11 / 2$ & 6714.184 & 474.692 & -29.633 & [7] \\
\hline & & & & 5042.526 & & 60 & $11 / 2$ & 6892.934 & 551.934 & -24.736 & {$[7]$} \\
\hline & & & & 5433.716 & $\mathrm{nl}$ & 14 & $9 / 2$ & 8320.240 & $255(2)$ & - & [19] \\
\hline & & & & 5729.066 & $\mathrm{nl}, \mathrm{e}$ & 6 & $11 / 2$ & 9268.726 & 977 (1) & $-24(20)$ & [19] \\
\hline & & & & 5800.466 & $\mathrm{nl}$ & 15 & $11 / 2$ & 9483.518 & $731(1)$ & $-15(10)$ & [19] \\
\hline & & & & 5875.89 & $\mathrm{nl}, \mathrm{e}$ & 1 & $7 / 2$ & 9704.744 & $779(1)$ & $-50(30)$ & [19] \\
\hline & & & & 5950.538 & $\mathrm{nl}$ & 13 & $7 / 2$ & 9918.190 & $1057.4(5)$ & $22(6)$ & {$[8]$} \\
\hline \multirow[t]{6}{*}{$7 / 2$} & 27096.093 & 918 (3) & & 4862.332 & $\mathrm{nl}, \mathrm{e}$ & 12 & $7 / 2$ & 6535.572 & $979(1)$ & $25(30)$ & [19] \\
\hline & & & & 5132.395 & $\mathrm{f}$ & 55 & $7 / 2$ & 7617.440 & 866.9 (5) & $-4(5)$ & {$[8]$} \\
\hline & & & & 5238.807 & $\mathrm{f}$ & 35 & $7 / 2$ & 8013.089 & $168(1)$ & - & This work \\
\hline & & & & 6470.79 & $\mathrm{nl}, \mathrm{e}$ & 1 & $5 / 2$ & 11646.312 & $1317(10)$ & - & [19] \\
\hline & & & & 6498.944 & nl, e & 15 & $9 / 2$ & 11713.236 & $818(2)$ & - & [19] \\
\hline & & & & 6652.28 & $\mathrm{nl}, \mathrm{e}$ & 1 & $9 / 2$ & 12067.802 & $873(6)$ & - & [19] \\
\hline \multirow[t]{10}{*}{$15 / 2$} & 27294.906 & $790(5)$ & & 5280.870 & $\mathrm{f}$ & 110 & $15 / 2$ & 8363.901 & 763.306 & -48.253 & {$[7]$} \\
\hline & & & & 5395.339 & $\mathrm{nl}$ & 12 & $15 / 2$ & 8765.542 & 763.557 & -45.805 & {$[7]$} \\
\hline & & & & 5606.822 & & 42 & $13 / 2$ & 9464.440 & $1056(1)$ & $-15(10)$ & [19] \\
\hline & & & & 5870.913 & & 140 & $13 / 2$ & 10266.501 & $972(2)$ & - & This work \\
\hline & & & & 5925.599 & & 44 & $13 / 2$ & 10423.654 & 869 (1) & $25(30)$ & This work \\
\hline & & & & 5942.039 & $\mathrm{nl}$ & 5 & $13 / 2$ & 10470.329 & $628(3)$ & - & [19] \\
\hline & & & & 6314.57 & nl, e & 1 & $13 / 2$ & 11462.895 & 804 (1) & - & This work \\
\hline & & & & 6322.77 & $\mathrm{nl}, \mathrm{e}$ & 1 & $15 / 2$ & 11483.427 & $987(1)$ & - & This work \\
\hline & & & & 7709.96 & $\mathrm{nl}, \mathrm{e}$ & 1 & $13 / 2$ & 14328.241 & $620(2)$ & $30(30)$ & {$[19]$} \\
\hline & & & & 9052.188 & & 8 & $13 / 2$ & 16250.885 & $892(1)$ & - & [19] \\
\hline
\end{tabular}


Table 5: Continued.

\begin{tabular}{|c|c|c|c|c|c|c|c|c|c|c|c|}
\hline \multirow[b]{2}{*}{$J$} & \multicolumn{3}{|c|}{ New upper odd level } & \multicolumn{3}{|c|}{ Line } & \multicolumn{5}{|c|}{ Combining known lower even level } \\
\hline & Energy $/ \mathrm{cm}^{-1}$ & $\mathrm{~A} / \mathrm{MHz}$ & $\mathrm{B} / \mathrm{MHz}$ & $\lambda / \AA$ & C. & SNR & J & Energy $/ \mathrm{cm}^{-1}$ & $\mathrm{~A} / \mathrm{MHz}$ & $\mathrm{B} / \mathrm{MHz}$ & Reference to col. 10,11 \\
\hline \multirow[t]{9}{*}{$9 / 2$} & 27394.138 & $792(3)$ & & 4634.502 & $\mathrm{nl}$ & 4 & $9 / 2$ & 5822.890 & $855.8(4)$ & $-17(7)$ & {$[8]$} \\
\hline & & & & 4742.301 & $\mathrm{f}$ & 55 & $11 / 2$ & 6313.224 & $756(1)$ & - & This work \\
\hline & & & & 4792.854 & $\mathrm{f}$ & 24 & $7 / 2$ & 6535.572 & $979(1)$ & $25(30)$ & [19] \\
\hline & & & & 4834.250 & $\mathrm{f}$ & 12 & $11 / 2$ & 6714.184 & 474.692 & -29.633 & [7] \\
\hline & & & & 5162.555 & $\mathrm{nl}$ & 11 & $9 / 2$ & 8029.275 & $797(2)$ & - & [19] \\
\hline & & & & 5331.761 & & 40 & $9 / 2$ & 8643.824 & $797(2)$ & - & [19] \\
\hline & & & & 6068.572 & & 12 & $9 / 2$ & 10920.365 & $632(2)$ & - & [19] \\
\hline & & & & 6470.73 & $\mathrm{nl}, \mathrm{e}$ & 1 & $11 / 2$ & 11944.207 & $1003(1)$ & - & This work \\
\hline & & & & 6522.91 & $\mathrm{nl}, \mathrm{e}$ & 1 & $9 / 2$ & 12067.802 & $873(6)$ & - & [19] \\
\hline \multirow[t]{11}{*}{$9 / 2$} & 27745.272 & $862(2)$ & & 4288.237 & $\mathrm{nl}$ & 11 & $9 / 2$ & 4432.225 & $923.2(4)$ & $-22(7)$ & [8] \\
\hline & & & & 4664.604 & $\mathrm{nl}, \mathrm{f}$ & 1 & $11 / 2$ & 6313.224 & $756(1)$ & - & This work \\
\hline & & & & 4753.538 & $\mathrm{nl}, \mathrm{f}$ & 5 & $11 / 2$ & 6714.184 & 474.692 & -29.633 & [7] \\
\hline & & & & 4794.287 & $\mathrm{f}$ & 7 & $11 / 2$ & 6892.934 & 551.934 & -24.736 & [7] \\
\hline & & & & 5233.749 & & 18 & $9 / 2$ & 8643.824 & $797(2)$ & - & [19] \\
\hline & & & & 5363.244 & $\mathrm{nl}, \mathrm{f}$ & 7 & $9 / 2$ & 9105.021 & $689.7(3)$ & $-3(5)$ & {$[8]$} \\
\hline & & & & 5474.405 & & 27 & $11 / 2$ & 9483.518 & $731(1)$ & $-15(10)$ & [19] \\
\hline & & & & 5696.259 & e & 18 & $7 / 2$ & 10194.768 & $855(1)$ & - & [19] \\
\hline & & & & 5749.322 & e & 25 & $9 / 2$ & 10356.737 & $1406(1)$ & - & This work \\
\hline & & & & 6218.547 & $\mathrm{nl}$ & 8 & $7 / 2$ & 11668.794 & $805(2)$ & - & [19] \\
\hline & & & & 6235.786 & $\mathrm{nl}$ & 5 & $9 / 2$ & 11713.236 & $818(2)$ & - & [19] \\
\hline \multirow[t]{8}{*}{$17 / 2$} & 27844.889 & $602(2)$ & $-40(50)$ & 5131.780 & $\mathrm{nl}$ & 7 & $15 / 2$ & 8363.901 & 763.306 & -48.253 & {$[7]$} \\
\hline & & & & 5239.809 & $\mathrm{f}$ & 30 & $15 / 2$ & 8765.542 & 763.557 & -45.805 & {$[7]$} \\
\hline & & & & 5752.739 & e & 75 & $15 / 2$ & 10466.689 & $1042(2)$ & $-20(30)$ & This work \\
\hline & & & & 5774.425 & e & 185 & $17 / 2$ & 10531.951 & $546(3)$ & - & [19] \\
\hline & & & & 6617.063 & $\mathrm{nl}$ & 4 & $17 / 2$ & 12736.621 & $904(1)$ & $20(20)$ & This work \\
\hline & & & & 6939.693 & & 5 & $15 / 2$ & 13439.009 & $916(3)$ & - & [19] \\
\hline & & & & 7235.894 & $\mathrm{nl}$ & 3 & $17 / 2$ & 14028.707 & $357(2)$ & - & [19] \\
\hline & & & & 7338.171 & $\mathrm{nl}$ & 4 & $15 / 2$ & 14221.272 & $345(1)$ & $-25(20)$ & [19] \\
\hline \multirow[t]{11}{*}{$15 / 2$} & 27950.530 & $824(1)$ & & 4683.201 & $\mathrm{nl}$ & 4 & $13 / 2$ & 6603.591 & 755.456 & -48.633 & [7] \\
\hline & & & & 5104.101 & & 35 & $15 / 2$ & 8363.901 & 763.306 & -48.253 & [7] \\
\hline & & & & 5210.956 & $\mathrm{f}$ & 130 & $15 / 2$ & 8765.542 & 763.557 & -45.805 & [7] \\
\hline & & & & 5407.968 & $\mathrm{f}$ & 45 & $13 / 2$ & 9464.440 & $1056(1)$ & $-15(10)$ & [19] \\
\hline & & & & 5491.426 & $\mathrm{nl}, \mathrm{f}$ & 25 & $15 / 2$ & 9745.376 & $540(2)$ & - & {$[4]$} \\
\hline & & & & 5703.941 & $\mathrm{nl}, \mathrm{e}$ & 12 & $13 / 2$ & 10423.654 & $869(1)$ & $25(30)$ & This work \\
\hline & & & & 5739.40 & $\mathrm{nl}, \mathrm{e}$ & 1 & $17 / 2$ & 10531.951 & $546(3)$ & - & [19] \\
\hline & & & & 6063.467 & $\mathrm{f}$ & 22 & $13 / 2$ & 11462.895 & $804(1)$ & - & This work \\
\hline & & & & 6100.423 & $\mathrm{f}$ & 30 & $13 / 2$ & 11562.762 & $819(2)$ & - & This work \\
\hline & & & & 6314.38 & $\mathrm{nl}, \mathrm{e}$ & 1 & $13 / 2$ & 12118.039 & $554(1)$ & $-45(30)$ & This work \\
\hline & & & & 6367.664 & $\mathrm{e}$ & 3 & $15 / 2$ & 12250.519 & $608(1)$ & - & This work \\
\hline \multirow[t]{11}{*}{$7 / 2$} & 28204.331 & $781(5)$ & & 4205.426 & $\mathrm{nl}, \mathrm{f}$ & 8 & $9 / 2$ & 4432.225 & $923.2(4)$ & $-22(7)$ & {$[8]$} \\
\hline & & & & 4466.734 & $\mathrm{nl}, \mathrm{f}$ & 9 & $9 / 2$ & 5822.890 & $855.8(4)$ & $-17(7)$ & {$[8]$} \\
\hline & & & & 4595.880 & $\mathrm{nl}, \mathrm{f}$ & 1 & $5 / 2$ & 6451.808 & $1189(6)$ & $-5(5)$ & {$[8]$} \\
\hline & & & & 4955.233 & $\mathrm{f}$ & 12 & $9 / 2$ & 8029.275 & $797(2)$ & - & [19] \\
\hline & & & & 5110.917 & $\mathrm{nl}, \mathrm{f}$ & 1 & $9 / 2$ & 8643.824 & $797(2)$ & - & [19] \\
\hline & & & & 5135.527 & $\mathrm{nl}, \mathrm{f}$ & 8 & $5 / 2$ & 8737.556 & 1149 (5) & - & [19] \\
\hline & & & & 5405.734 & $\mathrm{nl}$ & 8 & $5 / 2$ & 9710.600 & $164(2)$ & - & {$[19]$} \\
\hline & & & & 6467.24 & $\mathrm{nl}, \mathrm{e}$ & 1 & $9 / 2$ & 12746.067 & $982(1)$ & $10(10)$ & This work \\
\hline & & & & 6535.31 & nl, e & 1 & $5 / 2$ & 12907.057 & $1336(4)$ & - & This work \\
\hline & & & & 6630.96 & nl, e & 1 & $5 / 2$ & 13127.722 & $156(1)$ & $0(10)$ & [19] \\
\hline & & & & 7764.48 & $\mathrm{nl}, \mathrm{e}$ & 1 & $5 / 2$ & 15328.721 & $976(3)$ & - & [19] \\
\hline
\end{tabular}


Table 5: Continued.

\begin{tabular}{|c|c|c|c|c|c|c|c|c|c|c|c|}
\hline \multicolumn{4}{|c|}{ New upper odd level } & \multicolumn{3}{|c|}{ Line } & \multicolumn{5}{|c|}{ Combining known lower even level } \\
\hline$J$ & Energy $/ \mathrm{cm}^{-1}$ & $\mathrm{~A} / \mathrm{MHz}$ & $\mathrm{B} / \mathrm{MHz}$ & $\lambda / \AA$ & C. & SNR & $J$ & Energy $/ \mathrm{cm}^{-1}$ & $\mathrm{~A} / \mathrm{MHz}$ & $\mathrm{B} / \mathrm{MHz}$ & Reference to col. 10,11 \\
\hline \multirow[t]{9}{*}{$7 / 2$} & 28339.709 & $798(2)$ & & 4181.612 & $\mathrm{nl}$ & 11 & $9 / 2$ & 4432.225 & $923.2(4)$ & $-22(7)$ & {$[8]$} \\
\hline & & & & 4567.454 & $\mathrm{nl}$ & 4 & $5 / 2$ & 6451.808 & $1189(6)$ & $-5(5)$ & {$[8]$} \\
\hline & & & & 4918.284 & $\mathrm{nl}, \mathrm{f}$ & 1 & $7 / 2$ & 8013.089 & $168(1)$ & - & This work \\
\hline & & & & 5364.764 & $\mathrm{nl}$ & 9 & $7 / 2$ & 9704.744 & $779(1)$ & $-50(30)$ & [19] \\
\hline & & & & 5709.230 & nl, e & 12 & $5 / 2$ & 10829.070 & $980(1)$ & $-15(15)$ & [19] \\
\hline & & & & 5739.15 & $\mathrm{nl}, \mathrm{e}$ & 1 & $9 / 2$ & 10920.365 & $632(2)$ & - & [19] \\
\hline & & & & 5801.542 & $\mathrm{nl}$ & 15 & $5 / 2$ & 11107.696 & $658(2)$ & - & This work \\
\hline & & & & 5996.809 & $\mathrm{nl}, \mathrm{e}$ & 5 & $7 / 2$ & 11668.794 & $805(2)$ & - & [19] \\
\hline & & & & 6319.363 & $\mathrm{nl}$ & 7 & $9 / 2$ & 12519.705 & $693(3)$ & - & [19] \\
\hline \multirow[t]{12}{*}{$13 / 2$} & 28487.636 & $672(2)$ & & 4232.307 & $\mathrm{nl}$ & 10 & $11 / 2$ & 4866.515 & 867.997 & -50.319 & [7] \\
\hline & & & & 4508.438 & $\mathrm{nl}$ & 12 & $11 / 2$ & 6313.224 & $756(1)$ & - & This work \\
\hline & & & & 4568.259 & $\mathrm{nl}$ & 4 & $13 / 2$ & 6603.591 & 755.456 & -48.633 & [7] \\
\hline & & & & 4793.097 & & 4 & $13 / 2$ & 7630.132 & 776.286 & -43.592 & [7] \\
\hline & & & & 4967.870 & $\mathrm{f}$ & 60 & $15 / 2$ & 8363.901 & 763.306 & -48.253 & [7] \\
\hline & & & & 5201.762 & $\mathrm{f}$ & 30 & $11 / 2$ & 9268.726 & $977(1)$ & $-24(20)$ & [19] \\
\hline & & & & 5255.278 & $\mathrm{f}$ & 25 & $13 / 2$ & 9464.440 & $1056(1)$ & $-15(10)$ & [19] \\
\hline & & & & 5260.554 & & 18 & $11 / 2$ & 9483.518 & $731(1)$ & $-15(10)$ & [19] \\
\hline & & & & 5685.54 & $\mathrm{nl}, \mathrm{e}$ & 1 & $11 / 2$ & 10904.034 & $301(1)$ & $-20(10)$ & This work \\
\hline & & & & 5810.730 & $\mathrm{nl}, \mathrm{e}$ & 10 & $11 / 2$ & 11282.865 & 1049 (2) & - & This work \\
\hline & & & & 6315.68 & $\mathrm{nl}, \mathrm{e}$ & 1 & $11 / 2$ & 12658.401 & $995(5)$ & - & This work \\
\hline & & & & 6561.17 & $\mathrm{nl}$, e & 1 & $11 / 2$ & 13250.662 & $424(2)$ & - & [19] \\
\hline \multirow[t]{12}{*}{$7 / 2$} & 28525.358 & $843(2)$ & & 4403.570 & $\mathrm{nl}, \mathrm{f}$ & 9 & $9 / 2$ & 5822.890 & $855.8(4)$ & $-17(7)$ & {$[8]$} \\
\hline & & & & 4529.039 & $\mathrm{nl}$ & 8 & $5 / 2$ & 6451.808 & $1189(6)$ & $-5(5)$ & {$[8]$} \\
\hline & & & & 4546.291 & $\mathrm{nl}$ & 4 & $7 / 2$ & 6535.572 & $979(1)$ & $25(30)$ & [19] \\
\hline & & & & 4781.539 & & 10 & $7 / 2$ & 7617.440 & 866.9 (5) & $-4(5)$ & [8] \\
\hline & & & & 4873.768 & $\mathrm{nl}$ & 3 & $7 / 2$ & 8013.089 & $168(1)$ & - & This work \\
\hline & & & & 4877.62 & $\mathrm{nl}, \mathrm{f}$ & 1 & $9 / 2$ & 8029.275 & $797(2)$ & - & [19] \\
\hline & & & & 5028.391 & $\mathrm{nl}$ & 10 & $9 / 2$ & 8643.824 & $797(2)$ & - & [19] \\
\hline & & & & 5311.844 & $\mathrm{nl}$ & 7 & $7 / 2$ & 9704.744 & 779 (1) & $-50(30)$ & [19] \\
\hline & & & & 5453.846 & $\mathrm{f}$ & 27 & $7 / 2$ & 10194.768 & $855(1)$ & - & [19] \\
\hline & & & & 5739.705 & $\mathrm{nl}, \mathrm{e}$ & 5 & $5 / 2$ & 11107.696 & $658(2)$ & - & This work \\
\hline & & & & 5765.09 & $\mathrm{nl}, \mathrm{e}$ & 1 & $9 / 2$ & 11184.396 & $692(1)$ & $15(30)$ & This work \\
\hline & & & & 5795.12 & $\mathrm{nl}, \mathrm{e}$ & 1 & $7 / 2$ & 11274.229 & $1286(1)$ & $-10(20)$ & This work \\
\hline \multirow[t]{13}{*}{$9 / 2$} & 28579.596 & $765(2)$ & & 4934.613 & $\mathrm{nl}, \mathrm{f}$ & 1 & $9 / 2$ & 8320.240 & $255(2)$ & - & [19] \\
\hline & & & & 5014.710 & $\mathrm{nl}, \mathrm{f}$ & 10 & $9 / 2$ & 8643.824 & $797(2)$ & - & [19] \\
\hline & & & & 5061.743 & $\mathrm{f}$ & 22 & $11 / 2$ & 8829.063 & $769(1)$ & $-30(20)$ & This work \\
\hline & & & & 5176.989 & $\mathrm{nl}$ & 6 & $11 / 2$ & 9268.726 & $977(1)$ & $-24(20)$ & [19] \\
\hline & & & & 5288.254 & $\mathrm{nl}$ & 5 & $11 / 2$ & 9675.029 & $683(1)$ & - & [19] \\
\hline & & & & 5486.089 & $\mathrm{nl}, \mathrm{f}$ & 8 & $9 / 2$ & 10356.737 & $1406(1)$ & - & This work \\
\hline & & & & 5661.189 & $\mathrm{nl}, \mathrm{e}$ & 2 & $9 / 2$ & 10920.365 & $632(2)$ & - & [19] \\
\hline & & & & 5747.116 & nl, e & 15 & $9 / 2$ & 11184.396 & $692(1)$ & $15(30)$ & This work \\
\hline & & & & 5911.742 & $\mathrm{nl}$ & 15 & $7 / 2$ & 11668.794 & $805(2)$ & - & [19] \\
\hline & & & & 5927.318 & & 10 & $9 / 2$ & 11713.236 & $818(2)$ & - & [19] \\
\hline & & & & 6047.884 & & 18 & $7 / 2$ & 12049.465 & $871(2)$ & $-10(50)$ & [19] \\
\hline & & & & 6276.511 & $\mathrm{nl}$ & 6 & $9 / 2$ & 12651.586 & $723(3)$ & - & [19] \\
\hline & & & & 6755.704 & & 5 & $7 / 2$ & 13781.374 & 807 (1) & - & [19] \\
\hline
\end{tabular}


Table 5: Continued.

\begin{tabular}{|c|c|c|c|c|c|c|c|c|c|c|c|}
\hline \multirow[b]{2}{*}{$J$} & \multicolumn{3}{|c|}{ New upper odd level } & \multicolumn{3}{|c|}{ Line } & \multicolumn{5}{|c|}{ Combining known lower even level } \\
\hline & Energy $/ \mathrm{cm}^{-1}$ & $\mathrm{~A} / \mathrm{MHz}$ & $\mathrm{B} / \mathrm{MHz}$ & $\lambda / \AA ̊$ & C. & SNR & J & Energy $/ \mathrm{cm}^{-1}$ & $\mathrm{~A} / \mathrm{MHz}$ & $\mathrm{B} / \mathrm{MHz}$ & Reference to col. 10, 11 \\
\hline \multirow[t]{11}{*}{$15 / 2$} & 28754.446 & $639(2)$ & & 4513.233 & $\mathrm{nl}$ & 5 & $13 / 2$ & 6603.591 & 755.456 & -48.633 & {$[7]$} \\
\hline & & & & 4805.628 & & 4 & $13 / 2$ & 7951.323 & $644(1)$ & $-30(20)$ & [19] \\
\hline & & & & 5182.588 & & 30 & $13 / 2$ & 9464.440 & $1056(1)$ & $-15(10)$ & [19] \\
\hline & & & & 5266.081 & & 20 & $17 / 2$ & 9770.273 & 905.498 & -40.819 & [7] \\
\hline & & & & 5407.426 & $\mathrm{nl}$ & 8 & $13 / 2$ & 10266.501 & $972(2)$ & - & This work \\
\hline & & & & 5453.786 & & 22 & $13 / 2$ & 10423.654 & $869(1)$ & $25(30)$ & This work \\
\hline & & & & 5466.620 & $\mathrm{nl}, \mathrm{f}$ & 25 & $15 / 2$ & 10466.689 & $1042(2)$ & $-20(30)$ & This work \\
\hline & & & & 5467.709 & & 24 & $13 / 2$ & 10470.329 & $628(3)$ & - & [19] \\
\hline & & & & 5781.568 & $\mathrm{nl}, \mathrm{e}$ & 10 & $13 / 2$ & 11462.895 & $804(1)$ & - & This work \\
\hline & & & & 5788.44 & $\mathrm{nl}, \mathrm{e}$ & 1 & $15 / 2$ & 11483.427 & $987(1)$ & - & This work \\
\hline & & & & 7638.106 & $\mathrm{nl}$ & 3 & $17 / 2$ & 15665.796 & $906(1)$ & - & [19] \\
\hline \multirow[t]{4}{*}{$5 / 2$} & 28800.063 & $1057(2)$ & & 5220.453 & $\mathrm{f}$ & 60 & $3 / 2$ & 9649.970 & $1555(3)$ & - & [19] \\
\hline & & & & 5294.612 & $\mathrm{nl}$ & 12 & $7 / 2$ & 9918.190 & $1057.4(5)$ & $22(6)$ & {$[8]$} \\
\hline & & & & 5704.28 & $\mathrm{nl}, \mathrm{e}$ & 1 & $7 / 2$ & 11274.229 & $1286(1)$ & $-10(20)$ & This work \\
\hline & & & & 5769.52 & $\mathrm{nl}, \mathrm{e}$ & 1 & $7 / 2$ & 11472.410 & $273(3)$ & - & [19] \\
\hline \multirow[t]{10}{*}{$13 / 2$} & 28851.007 & $584(2)$ & & 4783.424 & & 25 & $13 / 2$ & 7951.323 & $644(1)$ & $-30(20)$ & [19] \\
\hline & & & & 5213.404 & & 100 & $11 / 2$ & 9675.029 & $683(1)$ & - & [19] \\
\hline & & & & 5232.600 & & 22 & $15 / 2$ & 9745.376 & $540(2)$ & - & {$[4]$} \\
\hline & & & & 5379.327 & $\mathrm{nl}$ & 6 & $13 / 2$ & 10266.501 & $972(2)$ & - & This work \\
\hline & & & & 5425.204 & $\mathrm{nl}$ & 18 & $13 / 2$ & 10423.654 & $869(1)$ & $25(30)$ & This work \\
\hline & & & & 5437.904 & $\mathrm{nl}, \mathrm{f}$ & 4 & $15 / 2$ & 10466.689 & $1042(2)$ & $-20(30)$ & This work \\
\hline & & & & 5690.540 & $\mathrm{nl}, \mathrm{e}$ & 32 & $11 / 2$ & 11282.865 & $1049(2)$ & - & This work \\
\hline & & & & 5749.46 & $\mathrm{nl}, \mathrm{e}$ & 1 & $13 / 2$ & 11462.895 & $804(1)$ & - & This work \\
\hline & & & & 5756.26 & $\mathrm{nl}, \mathrm{e}$ & 1 & $15 / 2$ & 11483.427 & $987(1)$ & - & This work \\
\hline & & & & 8062.490 & & 6 & $15 / 2$ & 16451.306 & $1037(3)$ & - & [19] \\
\hline \multirow[t]{8}{*}{$7 / 2$} & 28859.879 & $538(2)$ & & 4339.625 & $\mathrm{nl}, \mathrm{f}$ & 6 & $9 / 2$ & 5822.890 & $855.8(4)$ & $-17(7)$ & [8] \\
\hline & & & & 4706.240 & $\mathrm{nl}, \mathrm{f}$ & 1 & $7 / 2$ & 7617.440 & $866.9(5)$ & $-4(5)$ & [8] \\
\hline & & & & 4799.287 & $\mathrm{nl}, \mathrm{f}$ & 2 & $9 / 2$ & 8029.275 & $797(2)$ & - & [19] \\
\hline & & & & 5060.635 & $\mathrm{f}$ & 15 & $9 / 2$ & 9105.021 & $689.7(3)$ & $-3(5)$ & {$[8]$} \\
\hline & & & & 5219.079 & $\mathrm{nl}, \mathrm{f}$ & 1 & $7 / 2$ & 9704.744 & $779(1)$ & $-50(30)$ & [19] \\
\hline & & & & 5277.892 & $\mathrm{nl}, \mathrm{f}$ & 7 & $7 / 2$ & 9918.190 & $1057.4(5)$ & $22(6)$ & {$[8]$} \\
\hline & & & & 5749.67 & $\mathrm{nl}, \mathrm{e}$ & 1 & $7 / 2$ & 11472.410 & $273(3)$ & - & [19] \\
\hline & & & & 5807.763 & $\mathrm{nl}, \mathrm{e}$ & 10 & $5 / 2$ & 11646.312 & $1317(10)$ & - & [19] \\
\hline \multirow[t]{5}{*}{$5 / 2$} & 28920.891 & $1012(3)$ & & 4465.960 & $\mathrm{nl}, \mathrm{f}$ & 9 & $7 / 2$ & 6535.572 & $979(1)$ & $25(30)$ & [19] \\
\hline & & & & 5204.093 & & 15 & $5 / 2$ & 9710.600 & $164(2)$ & - & [19] \\
\hline & & & & 5665.220 & $\mathrm{nl}, \mathrm{e}$ & 5 & $7 / 2$ & 11274.229 & $1286(1)$ & $-10(20)$ & This work \\
\hline & & & & 5693.46 & $\mathrm{nl}, \mathrm{e}$ & 1 & $3 / 2$ & 11361.762 & $54(3)$ & - & [19] \\
\hline & & & & 7415.517 & $\mathrm{nl}, \mathrm{e}$ & 3 & $7 / 2$ & 15439.372 & $855(3)$ & - & [19] \\
\hline \multirow[t]{7}{*}{$13 / 2$} & 28921.386 & $697(2)$ & & 4959.956 & $\mathrm{f}$ & 8 & $15 / 2$ & 8765.542 & 763.557 & -45.805 & [7] \\
\hline & & & & 5143.164 & $\mathrm{nl}$ & 10 & $11 / 2$ & 9483.518 & $731(1)$ & $-15(10)$ & [19] \\
\hline & & & & 5418.237 & $\mathrm{nl}$ & 5 & $13 / 2$ & 10470.329 & $628(3)$ & - & [19] \\
\hline & & & & 5548.664 & $\mathrm{nl}$ & 5 & $11 / 2$ & 10904.034 & $301(1)$ & $-20(10)$ & This work \\
\hline & & & & 5667.837 & $\mathrm{nl}, \mathrm{e}$ & 5 & $11 / 2$ & 11282.865 & $1049(2)$ & - & This work \\
\hline & & & & 5726.28 & $\mathrm{nl}, \mathrm{e}$ & 1 & $13 / 2$ & 11462.895 & $804(1)$ & - & This work \\
\hline & & & & 5733.026 & $\mathrm{nl}, \mathrm{e}$ & 10 & $15 / 2$ & 11483.427 & $987(1)$ & - & This work \\
\hline \multirow[t]{4}{*}{$7 / 2$} & 28978.464 & $1047(3)$ & & 4072.794 & & 10 & $9 / 2$ & 4432.225 & $923.2(4)$ & $-22(7)$ & [8] \\
\hline & & & & 4437.939 & & 6 & $5 / 2$ & 6451.808 & $1189(6)$ & $-5(5)$ & [8] \\
\hline & & & & 4768.436 & $\mathrm{nl}$ & 1 & $7 / 2$ & 8013.089 & $168(1)$ & - & This work \\
\hline & & & & 4839.335 & $\mathrm{f}$ & 35 & $9 / 2$ & 8320.240 & $255(2)$ & - & [19] \\
\hline
\end{tabular}


Table 5: Continued.

\begin{tabular}{|c|c|c|c|c|c|c|c|c|c|c|c|}
\hline \multicolumn{4}{|c|}{ New upper odd level } & \multicolumn{3}{|c|}{ Line } & \multicolumn{5}{|c|}{ Combining known lower even level } \\
\hline \multirow[t]{4}{*}{$J$} & Energy $/ \mathrm{cm}^{-1}$ & $\mathrm{~A} / \mathrm{MHz}$ & $\mathrm{B} / \mathrm{MHz}$ & $\lambda / \AA$ & C. & SNR & $J$ & Energy $/ \mathrm{cm}^{-1}$ & $\mathrm{~A} / \mathrm{MHz}$ & $\mathrm{B} / \mathrm{MHz}$ & Reference to col. 10, 11 \\
\hline & & & & 5736.02 & $\mathrm{nl}, \mathrm{e}$ & 1 & $9 / 2$ & 11549.602 & $1064(2)$ & - & This work \\
\hline & & & & 5768.02 & $\mathrm{nl}, \mathrm{e}$ & 1 & $5 / 2$ & 11646.312 & $1317(10)$ & - & {$[19]$} \\
\hline & & & & 7477.73 & $\mathrm{nl}, \mathrm{e}$ & 1 & $5 / 2$ & 15609.100 & $1148(3)$ & - & [19] \\
\hline \multirow[t]{6}{*}{$5 / 2$} & 29075.845 & $829(2)$ & & 4435.258 & $\mathrm{f}$ & 8 & $7 / 2$ & 6535.572 & $979(1)$ & $25(30)$ & [19] \\
\hline & & & & 5160.890 & $\mathrm{nl}$ & 5 & $7 / 2$ & 9704.744 & $779(1)$ & $-50(30)$ & [19] \\
\hline & & & & 5294.835 & $\mathrm{nl}$ & 5 & $7 / 2$ & 10194.768 & $855(1)$ & - & [19] \\
\hline & & & & 5743.208 & $\mathrm{e}$ & 18 & $7 / 2$ & 11668.794 & $805(2)$ & - & [19] \\
\hline & & & & 5810.13 & $\mathrm{nl}$, e & 1 & $7 / 2$ & 11869.290 & $210(1)$ & - & [19] \\
\hline & & & & 5871.61 & $\mathrm{nl}, \mathrm{e}$ & 1 & $7 / 2$ & 12049.465 & $871(2)$ & $-10(50)$ & [19] \\
\hline \multirow[t]{10}{*}{$15 / 2$} & 29094.822 & $634(2)$ & & 4728.263 & $\mathrm{f}$ & 16 & $13 / 2$ & 7951.323 & $644(1)$ & $-30(20)$ & [19] \\
\hline & & & & 4917.638 & $\mathrm{nl}$ & 5 & $15 / 2$ & 8765.542 & 763.557 & -45.805 & [7] \\
\hline & & & & 5366.732 & & 33 & $15 / 2$ & 10466.689 & $1042(2)$ & $-20(30)$ & This work \\
\hline & & & & 5367.781 & $\mathrm{nl}$ & 9 & $13 / 2$ & 10470.329 & $628(3)$ & - & [19] \\
\hline & & & & 5669.96 & $\mathrm{nl}, \mathrm{e}$ & 1 & $13 / 2$ & 11462.895 & $804(1)$ & - & This work \\
\hline & & & & 5702.23 & $\mathrm{nl}, \mathrm{e}$ & 1 & $13 / 2$ & 11562.762 & $819(2)$ & - & This work \\
\hline & & & & 5751.989 & $\mathrm{nl}, \mathrm{e}$ & 12 & $17 / 2$ & 11714.352 & $970(1)$ & $-15(10)$ & This work \\
\hline & & & & 5935.079 & & 18 & $15 / 2$ & 12250.519 & $608(1)$ & - & This work \\
\hline & & & & 6111.447 & $\mathrm{nl}$ & 4 & $17 / 2$ & 12736.621 & $904(1)$ & $20(20)$ & This work \\
\hline & & & & 6136.902 & & 11 & $15 / 2$ & 12804.468 & $732(1)$ & - & This work \\
\hline \multirow{12}{*}{$9 / 2$} & 29149.204 & $730(1)$ & & 4116.998 & $\mathrm{nl}, \mathrm{f}$ & 2 & $11 / 2$ & 4866.515 & 867.997 & -50.319 & [7] \\
\hline & & & & 4285.798 & $\mathrm{nl}$ & 1 & $9 / 2$ & 5822.890 & $855.8(4)$ & $-17(7)$ & {$[8]$} \\
\hline & & & & 4377.825 & $\mathrm{nl}, \mathrm{e}$ & 8 & $11 / 2$ & 6313.224 & $756(1)$ & - & This work \\
\hline & & & & 4643.001 & $\mathrm{nl}$ & 8 & $7 / 2$ & 7617.440 & $866.9(5)$ & $-4(5)$ & [8] \\
\hline & & & & 4875.407 & $\mathrm{nl}, \mathrm{f}$ & 1 & $9 / 2$ & 8643.824 & 797 (2) & - & [19] \\
\hline & & & & 4919.852 & $\mathrm{nl}, \mathrm{f}$ & 18 & $11 / 2$ & 8829.063 & $769(1)$ & $-30(20)$ & This work \\
\hline & & & & 4987.587 & $\mathrm{nl}$ & 1 & $9 / 2$ & 9105.021 & $689.7(3)$ & $-3(5)$ & [8] \\
\hline & & & & 5028.688 & $\mathrm{nl}, \mathrm{f}$ & 1 & $11 / 2$ & 9268.726 & $977(1)$ & $-24(20)$ & [19] \\
\hline & & & & 5083.612 & $\mathrm{nl}$ & 1 & $11 / 2$ & 9483.518 & $731(1)$ & $-15(10)$ & [19] \\
\hline & & & & 5133.577 & $\mathrm{nl}$ & 19 & $11 / 2$ & 9675.029 & $683(1)$ & - & [19] \\
\hline & & & & 5785.463 & $\mathrm{nl}, \mathrm{e}$ & 10 & $7 / 2$ & 11869.290 & $210(1)$ & - & [19] \\
\hline & & & & 5810.65 & $\mathrm{nl}, \mathrm{e}$ & 1 & $11 / 2$ & 11944.207 & $1003(1)$ & - & This work \\
\hline \multirow[t]{12}{*}{$3 / 2$} & 29190.205 & $553(1)$ & & 4396.612 & $\mathrm{nl}, \mathrm{f}$ & 1 & $5 / 2$ & 6451.808 & $1189(6)$ & $-5(5)$ & {$[8]$} \\
\hline & & & & 4887.976 & $\mathrm{e}$ & 3 & $5 / 2$ & 8737.556 & $1149(5)$ & - & [19] \\
\hline & & & & 5116.221 & $\mathrm{nl}, \mathrm{f}$ & 1 & $3 / 2$ & 9649.970 & $1555(3)$ & - & [19] \\
\hline & & & & 5132.144 & $\mathrm{nl}, \mathrm{f}$ & 5 & $5 / 2$ & 9710.600 & $164(2)$ & - & [19] \\
\hline & & & & 5444.773 & $\mathrm{nl}, \mathrm{f}$ & 15 & $5 / 2$ & 10829.070 & $980(1)$ & $-15(15)$ & [19] \\
\hline & & & & 5528.670 & $\mathrm{nl}, \mathrm{f}$ & 1 & $5 / 2$ & 11107.696 & $658(2)$ & - & This work \\
\hline & & & & 5607.458 & $\mathrm{nl}, \mathrm{f}$ & 1 & $3 / 2$ & 11361.762 & $54(3)$ & - & [19] \\
\hline & & & & 5698.408 & $\mathrm{nl}, \mathrm{e}$ & 6 & $5 / 2$ & 11646.312 & $1317(10)$ & - & [19] \\
\hline & & & & 5833.12 & $\mathrm{nl}, \mathrm{e}$ & 1 & $3 / 2$ & 12051.488 & $1071(3)$ & $10(20)$ & This work \\
\hline & & & & 6350.60 & $\mathrm{e}$ & 1 & $5 / 2$ & 13448.016 & $825(1)$ & $25(30)$ & This work \\
\hline & & & & 6468.65 & $\mathrm{nl}, \mathrm{e}$ & 1 & $5 / 2$ & 13735.298 & $775(3)$ & $5(20)$ & [19] \\
\hline & & & & 6518.00 & $\mathrm{nl}, \mathrm{e}$ & 1 & $1 / 2$ & 13852.32 & $1218(8)$ & 0 & This work \\
\hline \multirow[t]{6}{*}{$11 / 2$} & 29195.032 & $805(2)$ & & 4705.959 & $\mathrm{f}$ & 15 & $13 / 2$ & 7951.323 & $644(1)$ & $-30(20)$ & [19] \\
\hline & & & & 5581.246 & $\mathrm{nl}, \mathrm{f}$ & 11 & $11 / 2$ & 11282.865 & $1049(2)$ & - & This work \\
\hline & & & & 5718.648 & $\mathrm{nl}, \mathrm{e}$ & 14 & $9 / 2$ & 11713.236 & $818(2)$ & - & {$[19]$} \\
\hline & & & & 5795.22 & $\mathrm{nl}, \mathrm{e}$ & 1 & $11 / 2$ & 11944.207 & $1003(1)$ & - & This work \\
\hline & & & & 5894.447 & e & 15 & $11 / 2$ & 12234.616 & $1169(2)$ & - & [19] \\
\hline & & & & 7826.958 & $\mathrm{nl}$ & 4 & $13 / 2$ & 16422.190 & $960(3)$ & - & [19] \\
\hline
\end{tabular}


Table 5: Continued.

\begin{tabular}{|c|c|c|c|c|c|c|c|c|c|c|c|}
\hline \multicolumn{4}{|c|}{ New upper odd level } & \multicolumn{3}{|c|}{ Line } & \multicolumn{5}{|c|}{ Combining known lower even level } \\
\hline$J$ & Energy $/ \mathrm{cm}^{-1}$ & $\mathrm{~A} / \mathrm{MHz}$ & $\mathrm{B} / \mathrm{MHz}$ & $\lambda / \AA$ & C. & SNR & $J$ & Energy $/ \mathrm{cm}^{-1}$ & $\mathrm{~A} / \mathrm{MHz}$ & $\mathrm{B} / \mathrm{MHz}$ & Reference to col. 10,11 \\
\hline \multirow[t]{5}{*}{$7 / 2$} & 29579.960 & $720(2)$ & & 4338.231 & $\mathrm{f}$ & 6 & $7 / 2$ & 6535.572 & $979(1)$ & $25(30)$ & {$[19]$} \\
\hline & & & & 4882.665 & e & 4 & $9 / 2$ & 9105.021 & $689.7(3)$ & $-3(5)$ & {$[8]$} \\
\hline & & & & 5029.989 & & 20 & $7 / 2$ & 9704.744 & $779(1)$ & $-50(30)$ & {$[19]$} \\
\hline & & & & 5702.76 & $\mathrm{nl}, \mathrm{e}$ & 1 & $7 / 2$ & 12049.465 & $871(2)$ & $-10(50)$ & [19] \\
\hline & & & & 5792.82 & nl, e & 1 & $7 / 2$ & 12321.991 & $871(3)$ & - & {$[19]$} \\
\hline \multirow[t]{9}{*}{$11 / 2$} & 29614.075 & $900(5)$ & & 4344.622 & $\mathrm{nl}$ & 5 & $13 / 2$ & 6603.591 & 755.456 & -48.633 & {$[7]$} \\
\hline & & & & 4365.606 & $\mathrm{nl}, \mathrm{f}$ & 1 & $11 / 2$ & 6714.184 & 474.692 & -29.633 & [7] \\
\hline & & & & 4614.926 & $\mathrm{nl}, \mathrm{f}$ & 17 & $13 / 2$ & 7951.323 & $644(1)$ & $-30(20)$ & {$[19]$} \\
\hline & & & & 4694.881 & $\mathrm{nl}, \mathrm{f}$ & 1 & $9 / 2$ & 8320.240 & $255(2)$ & - & [19] \\
\hline & & & & 4966.186 & $\mathrm{nl}, \mathrm{f}$ & 1 & $11 / 2$ & 9483.518 & $731(1)$ & $-15(10)$ & [19] \\
\hline & & & & 5167.167 & $\mathrm{nl}$ & 5 & $13 / 2$ & 10266.501 & $972(2)$ & - & This work \\
\hline & & & & 5538.225 & $\mathrm{nl}$ & 5 & $13 / 2$ & 11562.762 & $819(2)$ & - & This work \\
\hline & & & & 5697.63 & nl, e & 1 & $9 / 2$ & 12067.802 & $873(6)$ & - & [19] \\
\hline & & & & 5714.00 & $\mathrm{nl}, \mathrm{e}$ & 1 & $13 / 2$ & 12118.039 & $554(1)$ & $-45(30)$ & This work \\
\hline \multirow[t]{16}{*}{$17 / 2$} & 29622.951 & $501(1)$ & $15(20)$ & 5029.391 & & 20 & $15 / 2$ & 9745.376 & $540(2)$ & - & {$[4]$} \\
\hline & & & & 5035.700 & $\mathrm{nl}, \mathrm{f}$ & 8 & $17 / 2$ & 9770.273 & 905.498 & -40.819 & [7] \\
\hline & & & & 5218.772 & $\mathrm{nl}, \mathrm{f}$ & 1 & $15 / 2$ & 10466.689 & $1042(2)$ & $-20(30)$ & This work \\
\hline & & & & 5236.613 & $\mathrm{f}$ & 65 & $17 / 2$ & 10531.951 & $546(3)$ & - & [19] \\
\hline & & & & 5412.236 & $\mathrm{nl}, \mathrm{f}$ & 8 & $19 / 2$ & 11151.433 & $876.1(3)$ & $-31(12)$ & {$[8]$} \\
\hline & & & & 5511.292 & $\mathrm{nl}, \mathrm{f}$ & 1 & $15 / 2$ & 11483.427 & $987(1)$ & - & This work \\
\hline & & & & 5582.361 & $\mathrm{nl}$ & 50 & $17 / 2$ & 11714.352 & $970(1)$ & $-15(10)$ & This work \\
\hline & & & & 5754.650 & $\mathrm{e}$ & 75 & $15 / 2$ & 12250.519 & $608(1)$ & - & This work \\
\hline & & & & 5920.309 & e & 30 & $17 / 2$ & 12736.621 & 904 (1) & $20(20)$ & This work \\
\hline & & & & 6177.255 & $\mathrm{nl}, \mathrm{f}$ & 1 & $15 / 2$ & 13439.009 & $916(3)$ & - & [19] \\
\hline & & & & 6410.850 & $\mathrm{nl}, \mathrm{f}$ & 1 & $17 / 2$ & 14028.707 & $357(2)$ & - & [19] \\
\hline & & & & 6525.580 & $\mathrm{nl}, \mathrm{f}$ & 1 & $17 / 2$ & 14302.875 & $775(3)$ & - & [19] \\
\hline & & & & 6735.772 & $\mathrm{nl}, \mathrm{f}$ & 1 & $15 / 2$ & 14780.940 & $912(3)$ & - & [19] \\
\hline & & & & 6804.052 & $\mathrm{nl}, \mathrm{f}$ & 6 & $15 / 2$ & 14929.886 & $646(3)$ & - & [19] \\
\hline & & & & 7256.122 & $\mathrm{f}$ & 6 & $19 / 2$ & 15845.280 & $185(2)$ & - & [19] \\
\hline & & & & 9027.313 & $\mathrm{nl}$ & 4 & $15 / 2$ & 18548.497 & $573(5)$ & - & {$[4]$} \\
\hline \multirow[t]{13}{*}{$13 / 2$} & 29663.117 & $651(3)$ & & 4693.694 & $\mathrm{f}$ & 8 & $15 / 2$ & 8363.901 & 763.306 & -48.253 & [7] \\
\hline & & & & 4783.907 & & 4 & $15 / 2$ & 8765.542 & 763.557 & -45.805 & [7] \\
\hline & & & & 4901.942 & $\mathrm{nl}$ & 2 & $11 / 2$ & 9268.726 & 977 (1) & $-24(20)$ & [19] \\
\hline & & & & 4949.436 & & 2 & $13 / 2$ & 9464.440 & $1056(1)$ & $-15(10)$ & [19] \\
\hline & & & & 4954.177 & $\mathrm{nl}, \mathrm{f}$ & 1 & $11 / 2$ & 9483.518 & 731 (1) & $-15(10)$ & [19] \\
\hline & & & & 5001.586 & & 4 & $11 / 2$ & 9675.029 & $683(1)$ & - & [19] \\
\hline & & & & 5019.251 & & 65 & $15 / 2$ & 9745.376 & $540(2)$ & - & {$[4]$} \\
\hline & & & & 5196.203 & & 17 & $13 / 2$ & 10423.654 & 869 (1) & $25(30)$ & This work \\
\hline & & & & 5208.841 & $\mathrm{nl}$ & 13 & $13 / 2$ & 10470.329 & $628(3)$ & - & [19] \\
\hline & & & & 5329.268 & $\mathrm{nl}, \mathrm{f}$ & 1 & $11 / 2$ & 10904.034 & 301 (1) & $-20(10)$ & This work \\
\hline & & & & 5698.02 & $\mathrm{nl}, \mathrm{e}$ & 1 & $13 / 2$ & 12118.039 & $554(1)$ & $-45(30)$ & This work \\
\hline & & & & 5736.137 & $\mathrm{e}$ & 14 & $11 / 2$ & 12234.616 & $1169(2)$ & - & [19] \\
\hline & & & & 6244.930 & $\mathrm{nl}$ & 7 & $13 / 2$ & 13654.555 & $501(1)$ & $20(20)$ & [19] \\
\hline \multirow[t]{5}{*}{$7 / 2$} & 29675.405 & 865 (1) & & 4320.337 & & 14 & $7 / 2$ & 6535.572 & 979 (1) & $25(30)$ & [19] \\
\hline & & & & 5005.949 & $\mathrm{f}$ & 8 & $7 / 2$ & 9704.744 & 779 (1) & $-50(30)$ & [19] \\
\hline & & & & 5330.417 & $\mathrm{nl}$ & 9 & $9 / 2$ & 10920.365 & $632(2)$ & - & [19] \\
\hline & & & & 5671.88 & nl, e & 1 & $7 / 2$ & 12049.465 & $871(2)$ & $-10(50)$ & [19] \\
\hline & & & & 5681.28 & $\mathrm{nl}, \mathrm{e}$ & 1 & $5 / 2$ & 12078.621 & $1566(1)$ & - & [19] \\
\hline
\end{tabular}


Table 5: Continued.

\begin{tabular}{|c|c|c|c|c|c|c|c|c|c|c|c|}
\hline \multicolumn{4}{|c|}{ New upper odd level } & \multicolumn{3}{|c|}{ Line } & \multicolumn{5}{|c|}{ Combining known lower even level } \\
\hline$J$ & Energy $/ \mathrm{cm}^{-1}$ & $\mathrm{~A} / \mathrm{MHz}$ & $\mathrm{B} / \mathrm{MHz}$ & $\lambda / \AA$ & C. & SNR & $J$ & Energy $/ \mathrm{cm}^{-1}$ & $\mathrm{~A} / \mathrm{MHz}$ & $\mathrm{B} / \mathrm{MHz}$ & Reference to col. 10, 11 \\
\hline \multirow[t]{9}{*}{$13 / 2$} & 29774.999 & $615(2)$ & & 4580.896 & $\mathrm{nl}$ & 7 & $13 / 2$ & 7951.323 & $644(1)$ & $-30(20)$ & [19] \\
\hline & & & & 4758.430 & $\mathrm{f}$ & 10 & $15 / 2$ & 8765.542 & 763.557 & -45.805 & {$[7]$} \\
\hline & & & & 4973.743 & $\mathrm{nl}, \mathrm{e}$ & 4 & $11 / 2$ & 9675.029 & $683(1)$ & - & [19] \\
\hline & & & & 4991.212 & & 15 & $15 / 2$ & 9745.376 & $540(2)$ & - & [4] \\
\hline & & & & 5177.675 & & 15 & $15 / 2$ & 10466.689 & $1042(2)$ & $-20(30)$ & This work \\
\hline & & & & 5178.652 & $\mathrm{nl}$ & 15 & $13 / 2$ & 10470.329 & $628(3)$ & - & [19] \\
\hline & & & & 5661.92 & $\mathrm{nl}, \mathrm{e}$ & 1 & $13 / 2$ & 12118.039 & $554(1)$ & $-45(30)$ & This work \\
\hline & & & & 5681.90 & $\mathrm{nl}, \mathrm{e}$ & 1 & $11 / 2$ & 12180.131 & $679(3)$ & - & [19] \\
\hline & & & & 5704.72 & $\mathrm{nl}, \mathrm{e}$ & 1 & $15 / 2$ & 12250.519 & $608(1)$ & - & This work \\
\hline \multirow[t]{5}{*}{$3 / 2$} & 29804.574 & $1115(4)$ & & 5347.001 & $\mathrm{f}$ & 15 & $5 / 2$ & 11107.696 & $658(2)$ & - & This work \\
\hline & & & & 5420.660 & $\mathrm{nl}, \mathrm{f}$ & 1 & $3 / 2$ & 11361.762 & $54(3)$ & - & [19] \\
\hline & & & & 5699.767 & $\mathrm{nl}$ & 9 & $1 / 2$ & 12264.864 & $1534(2)$ & - & [19] \\
\hline & & & & 5916.390 & $\mathrm{nl}, \mathrm{e}$ & 1 & $5 / 2$ & 12907.057 & $1336(4)$ & - & This work \\
\hline & & & & 8035.00 & $\mathrm{nl}, \mathrm{e}$ & 1 & $5 / 2$ & 17362.452 & $451(3)$ & & [19] \\
\hline \multirow[t]{6}{*}{$13 / 2$} & 29836.950 & $535(2)$ & & 5388.150 & $\mathrm{nl}, \mathrm{f}$ & 10 & $11 / 2$ & 11282.865 & 1049 (2) & - & This work \\
\hline & & & & 5617.903 & $\mathrm{nl}$ & 1 & $13 / 2$ & 12041.655 & $1049(2)$ & - & This work \\
\hline & & & & 5642.124 & $\mathrm{nl}$ & 10 & $13 / 2$ & 12118.039 & $554(1)$ & $-45(30)$ & This work \\
\hline & & & & 5679.492 & $\mathrm{e}$ & 10 & $11 / 2$ & 12234.616 & $1169(2)$ & - & [19] \\
\hline & & & & 5684.625 & $\mathrm{nl}, \mathrm{e}$ & 6 & $15 / 2$ & 12250.519 & $608(1)$ & - & This work \\
\hline & & & & 6446.205 & $\mathrm{nl}$ & 3 & $13 / 2$ & 14328.241 & $620(2)$ & $30(30)$ & [19] \\
\hline \multirow[t]{7}{*}{$17 / 2$} & 29899.954 & $529(1)$ & & 5161.717 & $\mathrm{f}$ & 280 & $17 / 2$ & 10531.951 & $546(3)$ & - & [19] \\
\hline & & & & 5332.271 & $\mathrm{nl}, \mathrm{f}$ & 1 & $19 / 2$ & 11151.433 & $876.1(3)$ & $-31(12)$ & {$[8]$} \\
\hline & & & & 5428.396 & $\mathrm{nl}, \mathrm{f}$ & 9 & $15 / 2$ & 11483.427 & $987(1)$ & - & This work \\
\hline & & & & 5497.328 & $\mathrm{nl}, \mathrm{f}$ & 18 & $17 / 2$ & 11714.352 & $970(1)$ & $-15(10)$ & This work \\
\hline & & & & 5664.331 & $\mathrm{e}$ & 75 & $15 / 2$ & 12250.519 & $608(1)$ & - & This work \\
\hline & & & & 5847.876 & e & 10 & $15 / 2$ & 12804.468 & $732(1)$ & - & This work \\
\hline & & & & 6143.342 & $\mathrm{f}$ & 7 & $19 / 2$ & 13626.672 & $865(3)$ & - & [19] \\
\hline \multirow[t]{14}{*}{$15 / 2$} & 29942.851 & $583(2)$ & & 4632.848 & $\mathrm{nl}$ & 1 & $15 / 2$ & 8363.901 & 763.306 & -48.253 & [7] \\
\hline & & & & 4949.732 & $\mathrm{f}$ & 10 & $15 / 2$ & 9745.376 & $540(2)$ & - & [4] \\
\hline & & & & 5134.011 & $\mathrm{f}$ & 95 & $13 / 2$ & 10470.329 & $628(3)$ & - & [19] \\
\hline & & & & 5150.310 & $\mathrm{f}$ & 105 & $17 / 2$ & 10531.951 & $546(3)$ & - & [19] \\
\hline & & & & 5439.158 & $\mathrm{nl}, \mathrm{f}$ & 1 & $13 / 2$ & 11562.762 & $819(2)$ & - & This work \\
\hline & & & & 5484.391 & $\mathrm{nl}, \mathrm{f}$ & 1 & $17 / 2$ & 11714.352 & $970(1)$ & $-15(10)$ & This work \\
\hline & & & & 5584.666 & $\mathrm{nl}, \mathrm{f}$ & 5 & $13 / 2$ & 12041.655 & 1049 (2) & - & This work \\
\hline & & & & 5608.601 & $\mathrm{nl}, \mathrm{f}$ & 15 & $13 / 2$ & 12118.039 & $554(1)$ & $-45(30)$ & This work \\
\hline & & & & 5650.598 & nl, e & 10 & $15 / 2$ & 12250.519 & $608(1)$ & - & This work \\
\hline & & & & 5810.239 & $\mathrm{nl}, \mathrm{e}$ & 7 & $17 / 2$ & 12736.621 & $904(1)$ & $20(20)$ & This work \\
\hline & & & & 5833.24 & $\mathrm{nl}, \mathrm{e}$ & 1 & $15 / 2$ & 12804.468 & $732(1)$ & - & This work \\
\hline & & & & 6057.517 & $\mathrm{f}$ & 19 & $15 / 2$ & 13439.009 & $916(3)$ & - & [19] \\
\hline & & & & 6067.944 & $\mathrm{nl}$ & 10 & $13 / 2$ & 13467.373 & $599(1)$ & - & [19] \\
\hline & & & & 6305.292 & $\mathrm{nl}$ & 21 & $13 / 2$ & 14087.545 & $657(3)$ & - & [19] \\
\hline \multirow[t]{6}{*}{$19 / 2$} & 29950.715 & $418(2)$ & & 5482.025 & $\mathrm{f}$ & 85 & $17 / 2$ & 11714.352 & $970(1)$ & $-15(10)$ & This work \\
\hline & & & & 5783.441 & e & 11 & $21 / 2$ & 12664.765 & $825(2)$ & - & [19] \\
\hline & & & & 5807.582 & $\mathrm{e}$ & 12 & $17 / 2$ & 12736.621 & $904(1)$ & $20(20)$ & This work \\
\hline & & & & 6278.876 & $\mathrm{e}$ & 11 & $17 / 2$ & 14028.707 & $357(2)$ & - & [19] \\
\hline & & & & 6388.890 & & 8 & $17 / 2$ & 14302.875 & $775(3)$ & - & [19] \\
\hline & & & & 8640.361 & $\mathrm{nl}$ & 3 & $17 / 2$ & 18380.305 & $903(3)$ & - & [19] \\
\hline
\end{tabular}


Table 5: Continued.

\begin{tabular}{|c|c|c|c|c|c|c|c|c|c|c|c|}
\hline \multicolumn{4}{|c|}{ New upper odd level } & \multicolumn{3}{|c|}{ Line } & \multicolumn{5}{|c|}{ Combining known lower even level } \\
\hline$J$ & Energy $/ \mathrm{cm}^{-1}$ & $\mathrm{~A} / \mathrm{MHz}$ & $\mathrm{B} / \mathrm{MHz}$ & $\lambda / \AA$ & C. & SNR & $J$ & Energy/ $\mathrm{cm}^{-1}$ & $\mathrm{~A} / \mathrm{MHz}$ & $\mathrm{B} / \mathrm{MHz}$ & Reference to col. 10,11 \\
\hline \multirow[t]{8}{*}{$11 / 2$} & 29985.372 & $775(2)$ & & 4471.969 & $\mathrm{nl}$ & 2 & $13 / 2$ & 7630.132 & 776.286 & -43.592 & {$[7]$} \\
\hline & & & & 4537.158 & $\mathrm{nl}$ & 10 & $13 / 2$ & 7951.323 & $644(1)$ & $-30(20)$ & {$[19]$} \\
\hline & & & & 4876.245 & $\mathrm{nl}$ & 5 & $11 / 2$ & 9483.518 & $731(1)$ & $-15(10)$ & {$[19]$} \\
\hline & & & & 5069.870 & $\mathrm{f}$ & 18 & $13 / 2$ & 10266.501 & $972(2)$ & - & This work \\
\hline & & & & 5222.124 & $\mathrm{nl}, \mathrm{f}$ & 1 & $11 / 2$ & 10841.407 & $530(3)$ & - & [19] \\
\hline & & & & 5239.264 & $\mathrm{f}$ & 25 & $11 / 2$ & 10904.034 & $301(1)$ & $-20(10)$ & This work \\
\hline & & & & 5799.086 & $\mathrm{e}$ & 12 & $9 / 2$ & 12746.067 & $982(1)$ & $10(10)$ & This work \\
\hline & & & & 7848.97 & $\mathrm{nl}, \mathrm{e}$ & 1 & $13 / 2$ & 17248.351 & $775(3)$ & - & [19] \\
\hline \multirow[t]{9}{*}{$15 / 2$} & 29989.064 & $528(2)$ & & 4274.956 & $\mathrm{nl}$ & 13 & $13 / 2$ & 6603.591 & 755.456 & -48.633 & {$[7]$} \\
\hline & & & & 5120.900 & & 12 & $15 / 2$ & 10466.689 & $1042(2)$ & $-20(30)$ & This work \\
\hline & & & & 5121.857 & & 20 & $13 / 2$ & 10470.329 & $628(3)$ & - & [19] \\
\hline & & & & 5396.267 & & 10 & $13 / 2$ & 11462.895 & $804(1)$ & - & This work \\
\hline & & & & 5570.287 & $\mathrm{f}$ & 38 & $13 / 2$ & 12041.655 & 1049 (2) & - & This work \\
\hline & & & & 5635.875 & & 36 & $15 / 2$ & 12250.519 & $608(1)$ & - & This work \\
\hline & & & & 5794.67 & $\mathrm{nl}, \mathrm{e}$ & 1 & $17 / 2$ & 12736.621 & $904(1)$ & $20(20)$ & This work \\
\hline & & & & 5817.55 & $\mathrm{nl}, \mathrm{e}$ & 1 & $15 / 2$ & 12804.468 & $732(1)$ & - & This work \\
\hline & & & & 6383.593 & & 20 & $13 / 2$ & 14328.241 & $620(2)$ & $30(30)$ & [19] \\
\hline \multirow[t]{10}{*}{$9 / 2$} & 30035.792 & $884(2)$ & & 4214.208 & $\mathrm{nl}$ & 29 & $11 / 2$ & 6313.224 & $756(1)$ & - & This work \\
\hline & & & & 4254.083 & $\mathrm{nl}$ & 4 & $7 / 2$ & 6535.572 & $979(1)$ & $25(30)$ & [19] \\
\hline & & & & 4319.77 & $\mathrm{nl}, \mathrm{e}$ & 1 & $11 / 2$ & 6892.934 & 551.934 & -24.736 & [7] \\
\hline & & & & 4459.377 & $\mathrm{nl}, \mathrm{f}$ & 1 & $7 / 2$ & 7617.440 & $866.9(5)$ & $-4(5)$ & {$[8]$} \\
\hline & & & & 4813.971 & $\mathrm{nl}, \mathrm{f}$ & 1 & $11 / 2$ & 9268.726 & $977(1)$ & $-24(20)$ & [19] \\
\hline & & & & 4917.212 & $\mathrm{nl}, \mathrm{f}$ & 1 & $7 / 2$ & 9704.744 & $779(1)$ & $-50(30)$ & [19] \\
\hline & & & & 5331.018 & $\mathrm{nl}, \mathrm{f}$ & 1 & $11 / 2$ & 11282.865 & 1049 (2) & - & This work \\
\hline & & & & 5456.237 & $\mathrm{nl}, \mathrm{f}$ & 5 & $9 / 2$ & 11713.236 & $818(2)$ & - & [19] \\
\hline & & & & 5707.45 & $\mathrm{nl}, \mathrm{e}$ & 1 & $9 / 2$ & 12519.705 & $693(3)$ & - & [19] \\
\hline & & & & 5739.56 & $\mathrm{nl}, \mathrm{e}$ & 1 & $7 / 2$ & 12617.700 & $883(2)$ & - & [19] \\
\hline \multirow[t]{9}{*}{$11 / 2$} & 30053.346 & 759 (1) & & 4211.092 & $\mathrm{nl}$ & 16 & $11 / 2$ & 6313.224 & $756(1)$ & - & This work \\
\hline & & & & 4523.203 & $\mathrm{nl}$ & 22 & $13 / 2$ & 7951.323 & $644(1)$ & $-30(20)$ & [19] \\
\hline & & & & 4855.627 & $\mathrm{e}$ & 6 & $13 / 2$ & 9464.440 & $1056(1)$ & $-15(10)$ & [19] \\
\hline & & & & 5052.454 & $\mathrm{nl}, \mathrm{f}$ & 1 & $13 / 2$ & 10266.501 & $972(2)$ & - & This work \\
\hline & & & & 5220.665 & $\mathrm{nl}, \mathrm{f}$ & 14 & $11 / 2$ & 10904.034 & $301(1)$ & $-20(10)$ & This work \\
\hline & & & & 5451.014 & $\mathrm{nl}$ & 11 & $9 / 2$ & 11713.236 & $818(2)$ & - & [19] \\
\hline & & & & 5701.741 & $\mathrm{e}$ & 20 & $9 / 2$ & 12519.705 & $693(3)$ & - & [19] \\
\hline & & & & 5747.20 & $\mathrm{nl}, \mathrm{e}$ & 1 & $11 / 2$ & 12658.401 & $995(5)$ & - & This work \\
\hline & & & & 5949.782 & $\mathrm{nl}, \mathrm{f}$ & 1 & $11 / 2$ & 13250.662 & $424(2)$ & - & [19] \\
\hline \multirow[t]{7}{*}{$11 / 2$} & 30094.834 & $825(2)$ & & 3895.616 & $\mathrm{nl}$ & 20 & $9 / 2$ & 4432.225 & $923.2(4)$ & $-22(7)$ & {$[8]$} \\
\hline & & & & 4255.709 & $\mathrm{nl}$ & 6 & $13 / 2$ & 6603.591 & 755.456 & -48.633 & [7] \\
\hline & & & & 5082.164 & & 10 & $13 / 2$ & 10423.654 & 869 (1) & $25(30)$ & This work \\
\hline & & & & 5314.286 & $\mathrm{nl}, \mathrm{f}$ & 1 & $11 / 2$ & 11282.865 & 1049 (2) & - & This work \\
\hline & & & & 5394.551 & $\mathrm{nl}, \mathrm{f}$ & 1 & $13 / 2$ & 11562.762 & $819(2)$ & - & This work \\
\hline & & & & 5733.53 & $\mathrm{nl}, \mathrm{e}$ & 1 & $11 / 2$ & 12658.401 & $995(5)$ & - & This work \\
\hline & & & & 5762.500 & $\mathrm{nl}, \mathrm{e}$ & 9 & $9 / 2$ & 12746.067 & $982(1)$ & $10(10)$ & This work \\
\hline \multirow[t]{7}{*}{$11 / 2$} & 30111.020 & $786(2)$ & & 4252.777 & $\mathrm{nl}, \mathrm{f}$ & 6 & $13 / 2$ & 6603.591 & 755.456 & -48.633 & [7] \\
\hline & & & & 4272.879 & $\mathrm{nl}, \mathrm{f}$ & 3 & $11 / 2$ & 6714.184 & 474.692 & -29.633 & [7] \\
\hline & & & & 4305.776 & $\mathrm{nl}, \mathrm{f}$ & 1 & $11 / 2$ & 6892.934 & 551.934 & -24.736 & [7] \\
\hline & & & & 4511.432 & $\mathrm{nl}, \mathrm{f}$ & 1 & $13 / 2$ & 7951.323 & $644(1)$ & $-30(20)$ & [19] \\
\hline & & & & 5077.984 & $\mathrm{nl}$ & 8 & $13 / 2$ & 10423.654 & 869 (1) & $25(30)$ & This work \\
\hline & & & & 5090.053 & $\mathrm{nl}$ & 5 & $13 / 2$ & 10470.329 & $628(3)$ & - & [19] \\
\hline & & & & 5309.716 & $\mathrm{nl}$ & 9 & $11 / 2$ & 11282.865 & 1049 (2) & - & This work \\
\hline
\end{tabular}


Table 5: Continued.

\begin{tabular}{|c|c|c|c|c|c|c|c|c|c|c|c|}
\hline \multicolumn{4}{|c|}{ New upper odd level } & \multicolumn{3}{|c|}{ Line } & \multicolumn{5}{|c|}{ Combining known lower even level } \\
\hline \multirow[t]{3}{*}{$J$} & Energy $/ \mathrm{cm}^{-1}$ & $\mathrm{~A} / \mathrm{MHz}$ & $\mathrm{B} / \mathrm{MHz}$ & $\lambda / \AA$ & C. & SNR & $J$ & Energy $/ \mathrm{cm}^{-1}$ & $\mathrm{~A} / \mathrm{MHz}$ & $\mathrm{B} / \mathrm{MHz}$ & Reference to col. 10,11 \\
\hline & & & & 5728.21 & $\mathrm{nl}, \mathrm{e}$ & 1 & $11 / 2$ & 12658.401 & $995(5)$ & - & This work \\
\hline & & & & 5757.13 & $\mathrm{nl}$, e & 1 & $9 / 2$ & 12746.067 & $982(1)$ & $10(10)$ & This work \\
\hline \multirow[t]{13}{*}{$13 / 2$} & 30284.567 & $681(2)$ & & 4273.830 & $\mathrm{nl}, \mathrm{f}$ & 1 & $11 / 2$ & 6892.934 & 551.934 & -24.736 & {$[7]$} \\
\hline & & & & 4476.374 & $\mathrm{nl}, \mathrm{f}$ & 1 & $13 / 2$ & 7951.323 & $644(1)$ & $-30(20)$ & {$[19]$} \\
\hline & & & & 4756.985 & $\mathrm{nl}, \mathrm{f}$ & 1 & $11 / 2$ & 9268.726 & $977(1)$ & $-24(20)$ & [19] \\
\hline & & & & 4850.767 & $\mathrm{e}$ & 6 & $11 / 2$ & 9675.029 & $683(1)$ & - & [19] \\
\hline & & & & 4867.379 & $\mathrm{f}$ & 12 & $15 / 2$ & 9745.376 & $540(2)$ & - & {$[4]$} \\
\hline & & & & 4994.092 & $\mathrm{nl}, \mathrm{f}$ & 4 & $13 / 2$ & 10266.501 & $972(2)$ & - & This work \\
\hline & & & & 5317.344 & $\mathrm{nl}, \mathrm{f}$ & 15 & $15 / 2$ & 11483.427 & $987(1)$ & - & This work \\
\hline & & & & 5450.940 & $\mathrm{nl}, \mathrm{f}$ & 1 & $11 / 2$ & 11944.207 & $1003(1)$ & - & This work \\
\hline & & & & 5480.058 & $\mathrm{nl}, \mathrm{f}$ & 1 & $13 / 2$ & 12041.655 & $1049(2)$ & - & This work \\
\hline & & & & 5538.643 & $\mathrm{nl}, \mathrm{f}$ & 1 & $11 / 2$ & 12234.616 & $1169(2)$ & - & [19] \\
\hline & & & & 5671.81 & $\mathrm{nl}, \mathrm{e}$ & 1 & $11 / 2$ & 12658.401 & $995(5)$ & - & This work \\
\hline & & & & 5719.21 & $\mathrm{nl}, \mathrm{e}$ & 1 & $15 / 2$ & 12804.468 & $732(1)$ & - & This work \\
\hline & & & & 5833.38 & nl, e & 1 & $13 / 2$ & 13146.584 & $1070(1)$ & $45(20)$ & This work \\
\hline \multirow[t]{8}{*}{$5 / 2$} & 30304.145 & $818(2)$ & & 4484.846 & $\mathrm{nl}, \mathrm{f}$ & 1 & $7 / 2$ & 8013.089 & $168(1)$ & - & This work \\
\hline & & & & 4854.534 & $\mathrm{nl}, \mathrm{e}$ & 4 & $5 / 2$ & 9710.600 & $164(2)$ & - & [19] \\
\hline & & & & 5277.699 & $\mathrm{nl}, \mathrm{f}$ & 5 & $3 / 2$ & 11361.762 & $54(3)$ & - & {$[19]$} \\
\hline & & & & 5477.134 & $\mathrm{nl}, \mathrm{f}$ & 8 & $3 / 2$ & 12051.488 & $1071(3)$ & $10(20)$ & This work \\
\hline & & & & 5661.86 & $\mathrm{nl}, \mathrm{e}$ & 1 & $5 / 2$ & 12646.996 & $892(2)$ & - & This work \\
\hline & & & & 5746.49 & nl, e & 1 & $5 / 2$ & 12907.057 & $1336(4)$ & - & This work \\
\hline & & & & 5788.492 & $\mathrm{nl}$ & 7 & $3 / 2$ & 13033.280 & $905(4)$ & - & This work \\
\hline & & & & 5919.58 & $\mathrm{nl}, \mathrm{e}$ & 1 & $7 / 2$ & 13415.739 & $797(2)$ & - & This work \\
\hline \multirow[t]{5}{*}{$17 / 2$} & 30346.200 & $518(1)$ & & 4852.82 & $\mathrm{nl}, \mathrm{e}$ & 3 & $15 / 2$ & 9745.376 & $540(2)$ & - & {$[4]$} \\
\hline & & & & 5208.303 & & 25 & $19 / 2$ & 11151.433 & $876.1(3)$ & $-31(12)$ & {$[8]$} \\
\hline & & & & 5677.152 & $\mathrm{nl}$ & 10 & $17 / 2$ & 12736.621 & $904(1)$ & $20(20)$ & This work \\
\hline & & & & 5699.110 & $e, f$ & 27 & $15 / 2$ & 12804.468 & $732(1)$ & - & This work \\
\hline & & & & 5913.005 & $\mathrm{nl}, \mathrm{e}, \mathrm{f}$ & 25 & $15 / 2$ & 13439.009 & $916(3)$ & - & {$[19]$} \\
\hline \multirow[t]{7}{*}{$11 / 2$} & 30347.477 & $700(3)$ & & 4791.616 & $\mathrm{nl}$ & 7 & $11 / 2$ & 9483.518 & $731(1)$ & $-15(10)$ & [19] \\
\hline & & & & 4836.005 & $\mathrm{f}$ & 22 & $11 / 2$ & 9675.029 & $683(1)$ & - & [19] \\
\hline & & & & 5029.500 & $\mathrm{f}$ & 20 & $13 / 2$ & 10470.329 & $628(3)$ & - & [19] \\
\hline & & & & 5293.851 & $\mathrm{nl}$ & 6 & $13 / 2$ & 11462.895 & 804 (1) & - & This work \\
\hline & & & & 5679.79 & $\mathrm{nl}, \mathrm{e}$ & 1 & $9 / 2$ & 12746.067 & $982(1)$ & $10(10)$ & This work \\
\hline & & & & 6430.416 & $\mathrm{nl}, \mathrm{e}$ & 3 & $11 / 2$ & 14800.680 & $618(2)$ & $-30(20)$ & [19] \\
\hline & & & & 6470.81 & $\mathrm{nl}, \mathrm{e}$ & 1 & $9 / 2$ & 14897.721 & $722(2)$ & - & This work \\
\hline \multirow[t]{13}{*}{$13 / 2$} & 30412.010 & $658(2)$ & & 4250.671 & $\mathrm{nl}, \mathrm{f}$ & 1 & $11 / 2$ & 6892.934 & 551.934 & -24.736 & [7] \\
\hline & & & & 4772.488 & $\mathrm{nl}, \mathrm{f}$ & 1 & $13 / 2$ & 9464.440 & $1056(1)$ & $-15(10)$ & [19] \\
\hline & & & & 5012.309 & $\mathrm{f}$ & 10 & $15 / 2$ & 10466.689 & $1042(2)$ & $-20(30)$ & This work \\
\hline & & & & 5281.546 & $\mathrm{nl}, \mathrm{f}$ & 1 & $15 / 2$ & 11483.427 & $987(1)$ & - & This work \\
\hline & & & & 5442.042 & $\mathrm{nl}, \mathrm{f}$ & 5 & $13 / 2$ & 12041.655 & 1049 (2) & - & This work \\
\hline & & & & 5483.374 & $\mathrm{nl}$ & 1 & $11 / 2$ & 12180.131 & $679(3)$ & - & [19] \\
\hline & & & & 5499.812 & $\mathrm{nl}, \mathrm{f}$ & 10 & $11 / 2$ & 12234.616 & $1169(2)$ & - & [19] \\
\hline & & & & 5753.37 & $\mathrm{nl}, \mathrm{f}$ & 1 & $11 / 2$ & 13035.697 & $796(3)$ & - & This work \\
\hline & & & & 5790.316 & $\mathrm{nl}, \mathrm{e}$ & 1 & $13 / 2$ & 13146.584 & $1070(1)$ & $45(20)$ & This work \\
\hline & & & & 5825.433 & $\mathrm{nl}, \mathrm{e}$ & 7 & $11 / 2$ & 13250.662 & $424(2)$ & - & [19] \\
\hline & & & & 5868.635 & $\mathrm{nl}, \mathrm{e}$ & 10 & $11 / 2$ & 13376.992 & $868(2)$ & - & [19] \\
\hline & & & & 5899.937 & $\mathrm{nl}, \mathrm{f}$ & 1 & $13 / 2$ & 13467.373 & $599(1)$ & - & [19] \\
\hline & & & & 6053.742 & $\mathrm{nl}$ & 5 & $13 / 2$ & 13897.874 & $900(3)$ & - & This work \\
\hline
\end{tabular}


Table 5: Continued.

\begin{tabular}{|c|c|c|c|c|c|c|c|c|c|c|c|}
\hline \multicolumn{4}{|c|}{ New upper odd level } & \multicolumn{3}{|c|}{ Line } & \multicolumn{5}{|c|}{ Combining known lower even level } \\
\hline$J$ & Energy $/ \mathrm{cm}^{-1}$ & $\mathrm{~A} / \mathrm{MHz}$ & $\mathrm{B} / \mathrm{MHz}$ & $\lambda / \AA$ & C. & SNR & $J$ & Energy/ $\mathrm{cm}^{-1}$ & $\mathrm{~A} / \mathrm{MHz}$ & $\mathrm{B} / \mathrm{MHz}$ & Reference to col. 10,11 \\
\hline \multirow[t]{4}{*}{$21 / 2$} & 30543.982 & $392(2)$ & & 5155.184 & $\mathrm{nl}, \mathrm{f}$ & 11 & $19 / 2$ & 11151.433 & $876.1(3)$ & $-31(12)$ & {$[8]$} \\
\hline & & & & 5591.534 & $\mathrm{f}$ & 90 & $21 / 2$ & 12664.765 & $825(2)$ & - & {$[19]$} \\
\hline & & & & 5909.466 & e & 35 & $19 / 2$ & 13626.672 & $865(3)$ & - & {$[19]$} \\
\hline & & & & 6801.441 & e & 15 & $19 / 2$ & 15845.280 & $185(2)$ & - & [19] \\
\hline \multirow[t]{8}{*}{$13 / 2$} & 30562.652 & $537(1)$ & & 4742.700 & $\mathrm{f}$ & 6 & $11 / 2$ & 9483.518 & $731(1)$ & $-15(10)$ & [19] \\
\hline & & & & 4925.667 & $\mathrm{nl}, \mathrm{f}$ & 1 & $13 / 2$ & 10266.501 & $972(2)$ & - & This work \\
\hline & & & & 4964.105 & $\mathrm{f}$ & 6 & $13 / 2$ & 10423.654 & 869 (1) & $25(30)$ & This work \\
\hline & & & & 5185.335 & $\mathrm{f}$ & 16 & $11 / 2$ & 11282.865 & 1049 (2) & - & This work \\
\hline & & & & 5454.607 & $\mathrm{nl}, \mathrm{f}$ & 10 & $11 / 2$ & 12234.616 & $1169(2)$ & - & [19] \\
\hline & & & & 5703.92 & $\mathrm{nl}, \mathrm{e}$ & 1 & $11 / 2$ & 13035.697 & $796(3)$ & - & This work \\
\hline & & & & 5740.23 & $\mathrm{nl}, \mathrm{e}$ & 1 & $13 / 2$ & 13146.584 & $1070(1)$ & $45(20)$ & This work \\
\hline & & & & 5999.018 & $\mathrm{nl}, \mathrm{f}$ & 1 & $13 / 2$ & 13897.874 & $900(3)$ & - & This work \\
\hline \multirow[t]{11}{*}{$13 / 2$} & 30644.652 & $628(2)$ & & 3878.157 & $\mathrm{nl}$ & 15 & $11 / 2$ & 4866.515 & 867.997 & -50.319 & [7] \\
\hline & & & & 4158.377 & $\mathrm{nl}$ & 5 & $13 / 2$ & 6603.591 & 755.456 & -48.633 & [7] \\
\hline & & & & 4343.862 & $\mathrm{nl}$ & 3 & $13 / 2$ & 7630.132 & 776.286 & -43.592 & [7] \\
\hline & & & & 4724.321 & $\mathrm{nl}$ & 4 & $11 / 2$ & 9483.518 & $731(1)$ & $-15(10)$ & [19] \\
\hline & & & & 4943.974 & $\mathrm{nl}, \mathrm{f}$ & 1 & $13 / 2$ & 10423.654 & 869 (1) & $25(30)$ & This work \\
\hline & & & & 4954.519 & $\mathrm{f}$ & 6 & $15 / 2$ & 10466.689 & $1042(2)$ & $-20(30)$ & This work \\
\hline & & & & 4955.416 & & 6 & $13 / 2$ & 10470.329 & $628(3)$ & - & [19] \\
\hline & & & & 5713.331 & $\mathrm{nl}$ & 9 & $13 / 2$ & 13146.584 & $1070(1)$ & $45(20)$ & This work \\
\hline & & & & 5789.57 & $\mathrm{nl}, \mathrm{e}$ & 5 & $11 / 2$ & 13376.992 & $868(2)$ & - & [19] \\
\hline & & & & 5810.44 & $\mathrm{nl}, \mathrm{e}$ & 1 & $15 / 2$ & 13439.009 & $916(3)$ & - & [19] \\
\hline & & & & 5884.15 & nl, e & 1 & $13 / 2$ & 13654.555 & $501(1)$ & $20(20)$ & [19] \\
\hline \multirow[t]{5}{*}{$5 / 2$} & 30679.627 & $933(2)$ & & 4766.274 & $\mathrm{f}$ & 8 & $7 / 2$ & 9704.744 & 779 (1) & $-50(30)$ & [19] \\
\hline & & & & 5107.932 & $\mathrm{nl}$ & 4 & $5 / 2$ & 11107.696 & $658(2)$ & - & This work \\
\hline & & & & 5488.378 & $\mathrm{nl}$ & 6 & $3 / 2$ & 12464.369 & $712(4)$ & - & [19] \\
\hline & & & & 5695.81 & $\mathrm{nl}, \mathrm{e}$ & 1 & $5 / 2$ & 13127.722 & $156(1)$ & $0(10)$ & [19] \\
\hline & & & & 5721.43 & nl, e & 1 & $3 / 2$ & 13206.325 & $256(2)$ & $0(15)$ & [19] \\
\hline \multirow[t]{6}{*}{$5 / 2$} & 30763.323 & $981(2)$ & & 4319.208 & $\mathrm{nl}$ & 7 & $7 / 2$ & 7617.440 & $866.9(5)$ & $-4(5)$ & {$[8]$} \\
\hline & & & & 4538.864 & $\mathrm{nl}, \mathrm{f}$ & 1 & $5 / 2$ & 8737.556 & $1149(5)$ & - & [19] \\
\hline & & & & 5303.009 & $\mathrm{nl}$ & 10 & $3 / 2$ & 11911.350 & $920(3)$ & - & [19] \\
\hline & & & & 5342.725 & $\mathrm{nl}$ & 12 & $3 / 2$ & 12051.488 & $1071(3)$ & $10(20)$ & This work \\
\hline & & & & 5668.78 & $\mathrm{nl}, \mathrm{e}$ & 1 & $5 / 2$ & 13127.722 & $156(1)$ & $0(10)$ & [19] \\
\hline & & & & 5694.15 & $\mathrm{nl}, \mathrm{e}$ & 1 & $3 / 2$ & 13206.325 & $256(2)$ & $0(15)$ & [19] \\
\hline \multirow[t]{6}{*}{$15 / 2$} & 30788.090 & $528(2)$ & & 4919.547 & $\mathrm{nl}, \mathrm{f}$ & 1 & $15 / 2$ & 10466.689 & $1042(2)$ & $-20(30)$ & This work \\
\hline & & & & 5762.395 & $\mathrm{e}$ & 6 & $15 / 2$ & 13439.009 & $916(3)$ & - & [19] \\
\hline & & & & 5834.890 & nl, e & 7 & $13 / 2$ & 13654.555 & $501(1)$ & $20(20)$ & [19] \\
\hline & & & & 7071.443 & $\mathrm{nl}$ & 4 & $15 / 2$ & 16650.602 & $904(1)$ & $45(20)$ & [19] \\
\hline & & & & 7391.172 & $\mathrm{nl}$ & 5 & $15 / 2$ & 17262.152 & $905(3)$ & - & [19] \\
\hline & & & & 7520.285 & $\mathrm{nl}, \mathrm{e}$ & 5 & $17 / 2$ & 17494.382 & $468(3)$ & - & [19] \\
\hline \multirow[t]{8}{*}{$9 / 2$} & 30896.170 & $733(2)$ & & 3987.182 & $\mathrm{nl}, \mathrm{f}$ & 7 & $9 / 2$ & 5822.890 & $855.8(4)$ & $-17(7)$ & {$[8]$} \\
\hline & & & & 4371.907 & $\mathrm{nl}, \mathrm{f}$ & 5 & $9 / 2$ & 8029.275 & 797 (2) & - & [19] \\
\hline & & & & 4492.645 & $\mathrm{nl}, \mathrm{f}$ & 3 & $9 / 2$ & 8643.824 & 797 (2) & - & [19] \\
\hline & & & & 4622.460 & $\mathrm{nl}, \mathrm{f}$ & 1 & $11 / 2$ & 9268.726 & 977 (1) & $-24(20)$ & [19] \\
\hline & & & & 5211.516 & $\mathrm{f}$ & 20 & $9 / 2$ & 11713.236 & $818(2)$ & - & [19] \\
\hline & & & & 5781.92 & $\mathrm{nl}, \mathrm{e}$ & 1 & $9 / 2$ & 13605.665 & $681(2)$ & - & This work \\
\hline & & & & 5841.280 & $\mathrm{nl}, \mathrm{e}$ & 6 & $7 / 2$ & 13781.374 & 807 (1) & - & [19] \\
\hline & & & & 5855.35 & $\mathrm{nl}, \mathrm{e}$ & 1 & $9 / 2$ & 13822.494 & $1050(2)$ & - & \\
\hline
\end{tabular}


Table 5: Continued.

\begin{tabular}{|c|c|c|c|c|c|c|c|c|c|c|c|}
\hline \multicolumn{4}{|c|}{ New upper odd level } & \multicolumn{3}{|c|}{ Line } & \multicolumn{5}{|c|}{ Combining known lower even level } \\
\hline$J$ & Energy $/ \mathrm{cm}^{-1}$ & $\mathrm{~A} / \mathrm{MHz}$ & $\mathrm{B} / \mathrm{MHz}$ & $\lambda / \AA$ & C. & SNR & $J$ & Energy $/ \mathrm{cm}^{-1}$ & $\mathrm{~A} / \mathrm{MHz}$ & $\mathrm{B} / \mathrm{MHz}$ & Reference to col. 10,11 \\
\hline \multirow[t]{10}{*}{$9 / 2$} & 30901.231 & $730(2)$ & & 4293.625 & $\mathrm{nl}$, e & 1 & $7 / 2$ & 7617.440 & $866.9(5)$ & $-4(5)$ & {$[8]$} \\
\hline & & & & 4370.938 & $\mathrm{nl}, \mathrm{f}$ & 9 & $9 / 2$ & 8029.275 & $797(2)$ & - & {$[19]$} \\
\hline & & & & 4491.623 & $\mathrm{nl}, \mathrm{f}$ & 4 & $9 / 2$ & 8643.824 & $797(2)$ & - & {$[19]$} \\
\hline & & & & 4621.378 & $\mathrm{nl}, \mathrm{f}$ & 1 & $11 / 2$ & 9268.726 & $977(1)$ & $-24(20)$ & {$[19]$} \\
\hline & & & & 5210.141 & $\mathrm{nl}, \mathrm{f}$ & 1 & $9 / 2$ & 11713.236 & $818(2)$ & - & {$[19]$} \\
\hline & & & & 5273.622 & $\mathrm{nl}, \mathrm{f}$ & 1 & $11 / 2$ & 11944.207 & $1003(1)$ & - & This work \\
\hline & & & & 5308.229 & $\mathrm{nl}, \mathrm{f}$ & 10 & $9 / 2$ & 12067.802 & $873(6)$ & - & [19] \\
\hline & & & & 5780.229 & $\mathrm{nl}, \mathrm{e}$ & 7 & $9 / 2$ & 13605.665 & $681(2)$ & - & This work \\
\hline & & & & 5821.23 & $\mathrm{nl}$, e & 1 & $11 / 2$ & 13727.482 & 949 (1) & $-30(20)$ & [19] \\
\hline & & & & 5839.55 & $\mathrm{nl}, \mathrm{e}$ & 1 & $7 / 2$ & 13781.374 & $807(1)$ & - & [19] \\
\hline \multirow[t]{11}{*}{$7 / 2$} & 30975.616 & $734(2)$ & & 5031.837 & $\mathrm{nl}, \mathrm{f}$ & 12 & $5 / 2$ & 11107.696 & $658(2)$ & - & This work \\
\hline & & & & 5051.337 & $\mathrm{f}$ & 12 & $9 / 2$ & 11184.396 & $692(1)$ & $15(30)$ & This work \\
\hline & & & & 5074.370 & $\mathrm{nl}, \mathrm{f}$ & 1 & $7 / 2$ & 11274.229 & $1286(1)$ & $-10(20)$ & This work \\
\hline & & & & 5172.051 & $\mathrm{nl}$ & 4 & $5 / 2$ & 11646.312 & 1317 (10) & - & [19] \\
\hline & & & & 5232.415 & $\mathrm{nl}$ & 6 & $7 / 2$ & 11869.290 & $210(1)$ & - & [19] \\
\hline & & & & 5405.685 & $\mathrm{nl}$ & 8 & $5 / 2$ & 12481.714 & $937(1)$ & $20(20)$ & {$[19]$} \\
\hline & & & & 5445.728 & $\mathrm{nl}, \mathrm{f}$ & 1 & $7 / 2$ & 12617.700 & $883(2)$ & - & [19] \\
\hline & & & & 5703.705 & $\mathrm{nl}, \mathrm{e}$ & 6 & $5 / 2$ & 13448.016 & $825(1)$ & $25(30)$ & This work \\
\hline & & & & 5755.47 & $\mathrm{nl}, \mathrm{e}$ & 1 & $9 / 2$ & 13605.665 & $681(2)$ & - & This work \\
\hline & & & & 5776.93 & $\mathrm{nl}, \mathrm{e}$ & 1 & $5 / 2$ & 13670.175 & $18 \mathrm{v}$ & - & Be99v \\
\hline & & & & 5843.45 & $\mathrm{nl}, \mathrm{e}$ & 1 & $7 / 2$ & 13867.177 & $740(3)$ & - & This work \\
\hline \multirow[t]{13}{*}{$13 / 2$} & 31005.761 & $586(2)$ & & 3824.580 & $\mathrm{nl}, \mathrm{f}$ & 2 & $11 / 2$ & 4866.515 & 867.997 & -50.319 & {$[7]$} \\
\hline & & & & 4096.840 & $\mathrm{nl}, \mathrm{f}$ & 1 & $13 / 2$ & 6603.591 & 755.456 & -48.633 & {$[7]$} \\
\hline & & & & 4336.346 & $\mathrm{f}$ & 5 & $13 / 2$ & 7951.323 & $644(1)$ & $-30(20)$ & [19] \\
\hline & & & & 4495.097 & $\mathrm{nl}, \mathrm{f}$ & 3 & $15 / 2$ & 8765.542 & 763.557 & -45.805 & {$[7]$} \\
\hline & & & & 4645.053 & $\mathrm{nl}$ & 5 & $11 / 2$ & 9483.518 & $731(1)$ & $-15(10)$ & [19] \\
\hline & & & & 4867.409 & $\mathrm{f}$ & 15 & $15 / 2$ & 10466.689 & $1042(2)$ & $-20(30)$ & This work \\
\hline & & & & 4868.272 & $\mathrm{nl}, \mathrm{f}$ & 1 & $13 / 2$ & 10470.329 & $628(3)$ & - & [19] \\
\hline & & & & 5141.807 & $\mathrm{nl}, \mathrm{f}$ & 1 & $13 / 2$ & 11562.762 & 819 (2) & - & This work \\
\hline & & & & 5292.972 & $\mathrm{f}$ & 45 & $13 / 2$ & 12118.039 & $554(1)$ & $-45(30)$ & This work \\
\hline & & & & 5325.844 & $\mathrm{nl}, \mathrm{f}$ & 5 & $11 / 2$ & 12234.616 & $1169(2)$ & - & {$[19]$} \\
\hline & & & & 5563.265 & $\mathrm{nl}, \mathrm{f}$ & 1 & $11 / 2$ & 13035.697 & 796 (3) & - & This work \\
\hline & & & & 5761.69 & $\mathrm{nl}, \mathrm{e}$ & 1 & $13 / 2$ & 13654.555 & $501(1)$ & $20(20)$ & [19] \\
\hline & & & & 5834.90 & $\mathrm{nl}, \mathrm{e}$ & 5 & $11 / 2$ & 13872.266 & $872 \mathrm{v}$ & - & \\
\hline \multirow[t]{7}{*}{$15 / 2$} & 31039.972 & $553(1)$ & & 4091.104 & $\mathrm{f}$ & 25 & $13 / 2$ & 6603.591 & 755.456 & -48.633 & [7] \\
\hline & & & & 4694.712 & $\mathrm{nl}$ & 9 & $15 / 2$ & 9745.376 & $540(2)$ & - & {$[4]$} \\
\hline & & & & 4860.176 & f & 25 & $13 / 2$ & 10470.329 & $628(3)$ & - & [19] \\
\hline & & & & 4874.778 & & 7 & $17 / 2$ & 10531.951 & $546(3)$ & - & [19] \\
\hline & & & & 5320.654 & $\mathrm{nl}$ & 18 & $15 / 2$ & 12250.519 & $608(1)$ & - & This work \\
\hline & & & & 5689.099 & $\mathrm{nl}, \mathrm{e}$ & 20 & $13 / 2$ & 13467.373 & 599 (1) & - & [19] \\
\hline & & & & 5750.352 & $\mathrm{nl}, \mathrm{e}$ & 7 & $13 / 2$ & 13654.555 & $501(1)$ & $20(20)$ & [19] \\
\hline \multirow[t]{10}{*}{$17 / 2$} & 31270.847 & $512(2)$ & & 4364.261 & $\mathrm{nl}, \mathrm{f}$ & 1 & $15 / 2$ & 8363.901 & 763.306 & -48.253 & [7] \\
\hline & & & & 4442.150 & $\mathrm{nl}, \mathrm{f}$ & 1 & $15 / 2$ & 8765.542 & 763.557 & -45.805 & [7] \\
\hline & & & & 4649.737 & $\mathrm{nl}, \mathrm{f}$ & 1 & $17 / 2$ & 9770.273 & 905.498 & -40.819 & [7] \\
\hline & & & & 4820.511 & $\mathrm{f}$ & 5 & $17 / 2$ & 10531.951 & $546(3)$ & - & [19] \\
\hline & & & & 4968.937 & $\mathrm{f}$ & 12 & $19 / 2$ & 11151.433 & $876.1(3)$ & $-31(12)$ & {$[8]$} \\
\hline & & & & 5413.742 & $\mathrm{nl}$ & 8 & $15 / 2$ & 12804.468 & $732(1)$ & - & This work \\
\hline & & & & 5606.390 & $\mathrm{nl}, \mathrm{f}$ & 1 & $15 / 2$ & 13439.009 & $916(3)$ & - & {$[19]$} \\
\hline & & & & 5798.136 & $\mathrm{nl}, \mathrm{e}$ & 30 & $17 / 2$ & 14028.707 & 357 (2) & - & [19] \\
\hline & & & & 5863.62 & $\mathrm{nl}, \mathrm{e}$ & 1 & $15 / 2$ & 14221.272 & 345 (1) & $-25(20)$ & [19] \\
\hline & & & & 7256.759 & $\mathrm{nl}$ & 6 & $17 / 2$ & 17494.382 & $468(3)$ & - & [19] \\
\hline
\end{tabular}


Table 5: Continued.

\begin{tabular}{|c|c|c|c|c|c|c|c|c|c|c|c|}
\hline \multirow[b]{2}{*}{$J$} & \multicolumn{3}{|c|}{ New upper odd level } & \multicolumn{3}{|c|}{ Line } & \multicolumn{5}{|c|}{ Combining known lower even level } \\
\hline & Energy $/ \mathrm{cm}^{-1}$ & $\mathrm{~A} / \mathrm{MHz}$ & $\mathrm{B} / \mathrm{MHz}$ & $\lambda / \AA$ & C. & SNR & $J$ & Energy $/ \mathrm{cm}^{-1}$ & $\mathrm{~A} / \mathrm{MHz}$ & $\mathrm{B} / \mathrm{MHz}$ & Reference to col. 10, 11 \\
\hline \multirow[t]{9}{*}{$3 / 2$} & 31343.998 & $1123(2)$ & & 4422.278 & $\mathrm{nl}, \mathrm{f}$ & 4 & $5 / 2$ & 8737.556 & $1149(5)$ & - & {$[19]$} \\
\hline & & & & 4621.188 & $\mathrm{nl}, \mathrm{f}$ & 1 & $5 / 2$ & 9710.600 & $164(2)$ & - & [19] \\
\hline & & & & 5075.323 & $\mathrm{nl}, \mathrm{f}$ & 1 & $5 / 2$ & 11646.312 & $1317(10)$ & - & [19] \\
\hline & & & & 5181.915 & $\mathrm{nl}, \mathrm{f}$ & 1 & $3 / 2$ & 12051.488 & $1071(3)$ & $10(20)$ & This work \\
\hline & & & & 5239.869 & $\mathrm{nl}, \mathrm{f}$ & 1 & $1 / 2$ & 12264.864 & $1534(2)$ & - & [19] \\
\hline & & & & 5715.42 & $\mathrm{nl}, \mathrm{e}$ & 1 & $1 / 2$ & 13852.32 & $1218(8)$ & 0 & This work \\
\hline & & & & 5752.43 & $\mathrm{nl}, \mathrm{e}$ & 1 & $5 / 2$ & 13964.855 & $564(5)$ & $90(50)$ & [19] \\
\hline & & & & 5833.47 & $\mathrm{nl}, \mathrm{e}$ & 1 & $3 / 2$ & 14206.294 & $184(2)$ & - & {$[\mathrm{HH}]$} \\
\hline & & & & 6056.019 & $\mathrm{nl}$ & 6 & $5 / 2$ & 14836.072 & $1340(3)$ & - & {$[19]$} \\
\hline \multirow[t]{8}{*}{$9 / 2$} & 31447.902 & $665(2)$ & & 4591.588 & $\mathrm{nl}$ & 3 & $11 / 2$ & 9675.029 & $683(1)$ & - & [19] \\
\hline & & & & 4866.273 & $\mathrm{f}$ & 4 & $11 / 2$ & 10904.034 & $301(1)$ & $-20(10)$ & This work \\
\hline & & & & 4933.601 & $\mathrm{nl}$ & 4 & $9 / 2$ & 11184.396 & $692(1)$ & $15(30)$ & This work \\
\hline & & & & 5428.768 & $\mathrm{nl}$ & 7 & $7 / 2$ & 13032.634 & $893(3)$ & - & [19] \\
\hline & & & & 5544.107 & $\mathrm{nl}$ & 6 & $7 / 2$ & 13415.739 & $797(2)$ & - & This work \\
\hline & & & & 5744.87 & $\mathrm{nl}, \mathrm{e}$ & 1 & $7 / 2$ & 14045.878 & $463(2)$ & - & This work \\
\hline & & & & 5791.62 & $\mathrm{nl}, \mathrm{e}$ & 1 & $9 / 2$ & 14186.352 & $910(1)$ & $-60(30)$ & [19] \\
\hline & & & & 5887.789 & $\mathrm{e}$ & 5 & $9 / 2$ & 14468.303 & $762(2)$ & $20(20)$ & [19] \\
\hline \multirow[t]{6}{*}{$9 / 2$} & 31528.624 & $746(2)$ & & 4180.965 & $\mathrm{nl}$ & 10 & $7 / 2$ & 7617.440 & $866.9(5)$ & $-4(5)$ & {$[8]$} \\
\hline & & & & 5045.179 & $\mathrm{nl}$ & 7 & $9 / 2$ & 11713.236 & $818(2)$ & - & [19] \\
\hline & & & & 5405.078 & $\mathrm{nl}$ & 11 & $7 / 2$ & 13032.634 & $893(3)$ & - & [19] \\
\hline & & & & 5531.149 & $\mathrm{nl}, \mathrm{f}$ & 1 & $7 / 2$ & 13454.218 & $432(5)$ & - & [19] \\
\hline & & & & 5789.76 & $\mathrm{nl}, \mathrm{e}$ & 1 & $9 / 2$ & 14261.524 & $825(1)$ & $-30(20)$ & [19] \\
\hline & & & & 5793.565 & $\mathrm{e}$ & 13 & $9 / 2$ & 14272.877 & $731(3)$ & - & This work \\
\hline \multirow[t]{7}{*}{$7 / 2$} & 31668.068 & $852(2)$ & & 4229.144 & $\mathrm{nl}$ & 4 & $9 / 2$ & 8029.275 & $797(2)$ & - & [19] \\
\hline & & & & 4342.026 & $\mathrm{nl}$ & 4 & $9 / 2$ & 8643.824 & $797(2)$ & - & [19] \\
\hline & & & & 4969.170 & $\mathrm{nl}, \mathrm{f}$ & 1 & $9 / 2$ & 11549.602 & $1064(2)$ & - & This work \\
\hline & & & & 4993.174 & $\mathrm{nl}, \mathrm{f}$ & 1 & $5 / 2$ & 11646.312 & $1317(10)$ & - & [19] \\
\hline & & & & 5100.550 & $\mathrm{nl}, \mathrm{f}$ & 1 & $9 / 2$ & 12067.802 & $873(6)$ & - & {$[19]$} \\
\hline & & & & 5673.09 & $\mathrm{nl}, \mathrm{e}$ & 1 & $7 / 2$ & 14045.878 & $463(2)$ & - & This work \\
\hline & & & & 5747.12 & $\mathrm{nl}, \mathrm{e}$ & 1 & $9 / 2$ & 14272.877 & $731(3)$ & - & This work \\
\hline \multirow[t]{4}{*}{$15 / 2$} & 31668.549 & $544(2)$ & & 4729.807 & & 7 & $17 / 2$ & 10531.951 & $546(3)$ & - & [19] \\
\hline & & & & 5625.687 & $\mathrm{f}$ & 30 & $13 / 2$ & 13897.874 & $900(3)$ & - & This work \\
\hline & & & & 5729.96 & $\mathrm{nl}, \mathrm{e}$ & 1 & $15 / 2$ & 14221.272 & $345(1)$ & $-25(20)$ & [19] \\
\hline & & & & 5919.86 & $\mathrm{nl}, \mathrm{e}$ & 1 & $15 / 2$ & 14780.940 & $912(3)$ & - & [19] \\
\hline \multirow[t]{7}{*}{$15 / 2$} & 31817.886 & $437(2)$ & & 4911.429 & $\mathrm{nl}, \mathrm{f}$ & 1 & $13 / 2$ & 11462.895 & $804(1)$ & - & This work \\
\hline & & & & 4916.388 & $\mathrm{nl}, \mathrm{f}$ & 1 & $15 / 2$ & 11483.427 & $987(1)$ & - & This work \\
\hline & & & & 4972.862 & & 8 & $17 / 2$ & 11714.352 & $970(1)$ & $-15(10)$ & This work \\
\hline & & & & 5354.323 & & 32 & $13 / 2$ & 13146.584 & $1070(1)$ & $45(20)$ & This work \\
\hline & & & & 5447.928 & $\mathrm{nl}$ & 4 & $13 / 2$ & 13467.373 & $599(1)$ & - & [19] \\
\hline & & & & 5716.08 & $\mathrm{nl}, \mathrm{e}$ & 1 & $13 / 2$ & 14328.241 & $620(2)$ & $30(30)$ & [19] \\
\hline & & & & 5762.81 & $\mathrm{nl}, \mathrm{e}$ & 1 & $13 / 2$ & 14470.042 & $830(3)$ & - & [19] \\
\hline \multirow[t]{5}{*}{$11 / 2$} & 31895.205 & $668(2)$ & & 3698.720 & $\mathrm{f}$ & 10 & $11 / 2$ & 4866.515 & 867.997 & -50.319 & [7] \\
\hline & & & & 5054.927 & & 30 & $13 / 2$ & 12118.039 & $554(1)$ & $-45(30)$ & This work \\
\hline & & & & 5910.73 & $\mathrm{nl}, \mathrm{e}$ & 1 & $11 / 2$ & 14981.500 & $687(1)$ & - & [19] \\
\hline & & & & 5923.09 & $\mathrm{nl}, \mathrm{e}$ & 1 & $9 / 2$ & 15016.789 & 719.2 & - & $\mathrm{Bh}$ \\
\hline & & & & 5938.70 & $\mathrm{nl}, \mathrm{e}$ & 1 & $11 / 2$ & 15061.167 & $930(4)$ & - & [19] \\
\hline
\end{tabular}


Table 5: Continued.

\begin{tabular}{|c|c|c|c|c|c|c|c|c|c|c|c|}
\hline \multirow[b]{2}{*}{$J$} & \multicolumn{3}{|c|}{ New upper odd level } & \multicolumn{3}{|c|}{ Line } & \multicolumn{5}{|c|}{ Combining known lower even level } \\
\hline & Energy $/ \mathrm{cm}^{-1}$ & $\mathrm{~A} / \mathrm{MHz}$ & $\mathrm{B} / \mathrm{MHz}$ & $\lambda / \AA$ & C. & SNR & $J$ & Energy $/ \mathrm{cm}^{-1}$ & $\mathrm{~A} / \mathrm{MHz}$ & $\mathrm{B} / \mathrm{MHz}$ & Reference to col. 10, 11 \\
\hline \multirow[t]{5}{*}{$11 / 2$} & 32289.455 & $665(1)$ & & 5286.050 & $\mathrm{nl}$ & 8 & $11 / 2$ & 13376.992 & $868(2)$ & - & {$[19]$} \\
\hline & & & & 5458.571 & $\mathrm{nl}, \mathrm{f}$ & 15 & $9 / 2$ & 13974.732 & $854(1)$ & $-15(15)$ & [19] \\
\hline & & & & 5716.368 & $\mathrm{nl}, \mathrm{e}$ & 10 & $11 / 2$ & 14800.680 & $618(2)$ & $-30(20)$ & [19] \\
\hline & & & & 5806.88 & $\mathrm{nl}, \mathrm{e}$ & 1 & $13 / 2$ & 15073.268 & $756(2)$ & - & [19] \\
\hline & & & & 7586.509 & & 10 & $13 / 2$ & 19111.800 & $762(3)$ & - & This work \\
\hline \multirow[t]{6}{*}{$11 / 2$} & 32513.960 & $655(2)$ & & 4535.186 & $\mathrm{nl}, \mathrm{f}$ & 5 & $13 / 2$ & 10470.329 & $628(3)$ & - & [19] \\
\hline & & & & 4749.025 & $\mathrm{nl}, \mathrm{f}$ & 1 & $13 / 2$ & 11462.895 & $804(1)$ & - & This work \\
\hline & & & & 4806.181 & $\mathrm{nl}$ & 7 & $9 / 2$ & 11713.236 & $818(2)$ & - & [19] \\
\hline & & & & 4901.572 & $\mathrm{f}$ & 6 & $13 / 2$ & 12118.039 & $554(1)$ & $-45(30)$ & This work \\
\hline & & & & 5643.92 & $\mathrm{nl}, \mathrm{e}$ & 1 & $11 / 2$ & 14800.680 & $618(2)$ & $-30(20)$ & [19] \\
\hline & & & & 5786.83 & $\mathrm{nl}, \mathrm{e}$ & 1 & $11 / 2$ & 15238.143 & $1006(7)$ & $25(15)$ & [19] \\
\hline \multirow[t]{11}{*}{$13 / 2$} & 32608.552 & $620(3)$ & & 3844.331 & $\mathrm{nl}, \mathrm{f}$ & 4 & $13 / 2$ & 6603.591 & 755.456 & -48.633 & [7] \\
\hline & & & & 4054.460 & $\mathrm{nl}, \mathrm{f}$ & 1 & $13 / 2$ & 7951.323 & $644(1)$ & $-30(20)$ & [19] \\
\hline & & & & 4515.808 & $\mathrm{nl}, \mathrm{f}$ & 2 & $13 / 2$ & 10470.329 & $628(3)$ & - & [19] \\
\hline & & & & 4687.869 & $\mathrm{nl}, \mathrm{f}$ & 1 & $11 / 2$ & 11282.865 & $1049(2)$ & - & This work \\
\hline & & & & 4727.781 & $\mathrm{nl}, \mathrm{f}$ & 1 & $13 / 2$ & 11462.895 & $804(1)$ & - & This work \\
\hline & & & & 4732.376 & $\mathrm{nl}, \mathrm{f}$ & 1 & $15 / 2$ & 11483.427 & $987(1)$ & - & This work \\
\hline & & & & 4750.215 & $\mathrm{nl}, \mathrm{f}$ & 1 & $13 / 2$ & 11562.762 & $819(2)$ & - & This work \\
\hline & & & & 5136.795 & $\mathrm{nl}, \mathrm{f}$ & 1 & $13 / 2$ & 13146.584 & $1070(1)$ & $45(20)$ & This work \\
\hline & & & & 5701.20 & $\mathrm{nl}, \mathrm{e}$ & 1 & $13 / 2$ & 15073.268 & $756(2)$ & - & [19] \\
\hline & & & & 5728.26 & $\mathrm{nl}, \mathrm{e}$ & 1 & $13 / 2$ & 15156.070 & $1187(1)$ & - & This work \\
\hline & & & & 5800.11 & $\mathrm{nl}, \mathrm{e}$ & 1 & $13 / 2$ & 15372.271 & $642(1)$ & - & This work \\
\hline \multirow[t]{6}{*}{$15 / 2$} & 32672.439 & $601(2)$ & & 5014.698 & $\mathrm{nl}, \mathrm{f}$ & 14 & $17 / 2$ & 12736.621 & $904(1)$ & $20(20)$ & This work \\
\hline & & & & 5031.823 & $\mathrm{nl}, \mathrm{f}$ & 1 & $15 / 2$ & 12804.468 & $732(1)$ & - & This work \\
\hline & & & & 5492.256 & $\mathrm{nl}$ & 8 & $13 / 2$ & 14470.042 & $830(3)$ & - & [19] \\
\hline & & & & 5680.510 & $\mathrm{nl}, \mathrm{e}$ & 20 & $13 / 2$ & 15073.268 & $756(2)$ & - & {$[19]$} \\
\hline & & & & 5778.688 & $\mathrm{e}$ & 23 & $13 / 2$ & 15372.271 & $642(1)$ & - & This work \\
\hline & & & & 8296.640 & $\mathrm{nl}$ & 5 & $13 / 2$ & 20622.677 & $642(1)$ & $40(50)$ & This work \\
\hline \multirow[t]{3}{*}{$21 / 2$} & 32741.057 & $332(2)$ & & 4979.610 & & 8 & $21 / 2$ & 12664.765 & $825(2)$ & - & [19] \\
\hline & & & & 5230.205 & $\mathrm{f}$ & 60 & $19 / 2$ & 13626.672 & $865(3)$ & - & [19] \\
\hline & & & & 5917.00 & $\mathrm{nl}, \mathrm{e}$ & 1 & $19 / 2$ & 15845.280 & $185(2)$ & - & [19] \\
\hline \multirow[t]{9}{*}{$15 / 2$} & 32849.815 & $540(2)$ & & 4015.170 & $\mathrm{nl}$ & 4 & $13 / 2$ & 7951.323 & $644(1)$ & $-30(20)$ & [19] \\
\hline & & & & 4326.956 & $\mathrm{nl}, \mathrm{e}$ & 5 & $15 / 2$ & 9745.376 & $540(2)$ & - & {$[4]$} \\
\hline & & & & 4467.124 & $\mathrm{nl}, \mathrm{e}$ & 5 & $13 / 2$ & 10470.329 & $628(3)$ & - & [19] \\
\hline & & & & 4674.446 & $\mathrm{nl}, \mathrm{f}$ & 1 & $13 / 2$ & 11462.895 & $804(1)$ & - & This work \\
\hline & & & & 4696.376 & $\mathrm{nl}, \mathrm{f}$ & 1 & $13 / 2$ & 11562.762 & $819(2)$ & - & This work \\
\hline & & & & 5578.830 & $\mathrm{nl}, \mathrm{f}$ & 1 & $15 / 2$ & 14929.886 & $646(3)$ & - & [19] \\
\hline & & & & 5623.828 & $\mathrm{nl}, \mathrm{f}$ & 10 & $13 / 2$ & 15073.268 & $756(2)$ & - & [19] \\
\hline & & & & 5720.040 & $\mathrm{nl}, \mathrm{e}$ & 13 & $13 / 2$ & 15372.271 & $642(1)$ & - & This work \\
\hline & & & & 5735.74 & $\mathrm{nl}, \mathrm{e}$ & 1 & $13 / 2$ & 15420.120 & $1020(5)$ & - & This work \\
\hline \multirow[t]{7}{*}{$13 / 2$} & 33052.848 & $555(2)$ & & 3738.704 & $\mathrm{nl}$ & 7 & $11 / 2$ & 6313.224 & $756(1)$ & - & This work \\
\hline & & & & 3982.695 & $\mathrm{nl}, \mathrm{f}$ & 8 & $13 / 2$ & 7951.323 & $644(1)$ & $-30(20)$ & {$[19]$} \\
\hline & & & & 4789.605 & & 5 & $11 / 2$ & 12180.131 & $679(3)$ & - & [19] \\
\hline & & & & 4805.810 & & 6 & $15 / 2$ & 12250.519 & $608(1)$ & - & This work \\
\hline & & & & 5785.33 & $\mathrm{nl}, \mathrm{e}$ & 1 & $13 / 2$ & 15772.545 & $938(3)$ & - & [19] \\
\hline & & & & 5860.70 & $\mathrm{nl}, \mathrm{e}$ & 1 & $15 / 2$ & 15994.780 & $814(2)$ & - & [19] \\
\hline & & & & 5886.62 & $\mathrm{nl}, \mathrm{e}$ & 1 & $13 / 2$ & 16069.885 & $1050(3)$ & - & [19] \\
\hline
\end{tabular}


Table 5: Continued.

\begin{tabular}{|c|c|c|c|c|c|c|c|c|c|c|c|}
\hline \multicolumn{4}{|c|}{ New upper odd level } & \multicolumn{3}{|c|}{ Line } & \multicolumn{5}{|c|}{ Combining known lower even level } \\
\hline$J$ & Energy $/ \mathrm{cm}^{-1}$ & $\mathrm{~A} / \mathrm{MHz}$ & $\mathrm{B} / \mathrm{MHz}$ & $\lambda / \AA$ & C. & SNR & $J$ & Energy $/ \mathrm{cm}^{-1}$ & $\mathrm{~A} / \mathrm{MHz}$ & $\mathrm{B} / \mathrm{MHz}$ & Reference to col. 10,11 \\
\hline \multirow[t]{7}{*}{$17 / 2$} & 33305.483 & $450(2)$ & & 4389.829 & $\mathrm{nl}, \mathrm{e}$ & 1 & $17 / 2$ & 10531.951 & $546(3)$ & - & {$[19]$} \\
\hline & & & & 5775.171 & $\mathrm{nl}, \mathrm{f}$ & 10 & $15 / 2$ & 15994.780 & $814(2)$ & - & [19] \\
\hline & & & & 5876.880 & $\mathrm{f}$ & 16 & $15 / 2$ & 16294.378 & $825(1)$ & - & This work \\
\hline & & & & 6114.815 & $\mathrm{nl}, \mathrm{f}$ & 7 & $15 / 2$ & 16956.282 & $808(3)$ & - & [19] \\
\hline & & & & 6231.395 & $\mathrm{nl}, \mathrm{f}$ & 3 & $15 / 2$ & 17262.152 & $905(3)$ & - & [19] \\
\hline & & & & 6404.22 & $\mathrm{nl}, \mathrm{e}$ & 1 & $17 / 2$ & 17695.093 & $1059(3)$ & - & [19] \\
\hline & & & & 6470.68 & $\mathrm{nl}, \mathrm{e}$ & 1 & $17 / 2$ & 17855.428 & $947(2)$ & - & [19] \\
\hline \multirow[t]{3}{*}{$9 / 2$} & 33687.796 & $744(2)$ & & 3468.665 & $\mathrm{f}$ & 7 & $11 / 2$ & 4866.515 & 867.997 & -50.319 & [7] \\
\hline & & & & 5691.05 & $\mathrm{nl}, \mathrm{e}$ & 1 & $11 / 2$ & 16121.221 & $742(1)$ & $40(20)$ & [19] \\
\hline & & & & 5764.22 & $\mathrm{nl}, \mathrm{e}$ & 1 & $7 / 2$ & 16344.206 & $674(3)$ & - & [19] \\
\hline \multirow[t]{6}{*}{$13 / 2$} & 33932.700 & 737 (1) & & 3658.060 & $\mathrm{nl}$ & 6 & $13 / 2$ & 6603.591 & 755.456 & -48.633 & {$[7]$} \\
\hline & & & & 5165.634 & $\mathrm{nl}, \mathrm{f}$ & 6 & $15 / 2$ & 14579.390 & $873(3)$ & - & [19] \\
\hline & & & & 5260.914 & $\mathrm{nl}, \mathrm{f}$ & 66 & $15 / 2$ & 14929.886 & $646(3)$ & - & [19] \\
\hline & & & & 5667.90 & $\mathrm{nl}, \mathrm{e}$ & 1 & $15 / 2$ & 16294.378 & $825(1)$ & - & This work \\
\hline & & & & 5694.73 & $\mathrm{nl}, \mathrm{e}$ & 1 & $11 / 2$ & 16377.460 & $560(2)$ & - & This work \\
\hline & & & & 5709.27 & $\mathrm{nl}, \mathrm{e}$ & 1 & $13 / 2$ & 16422.190 & $960(3)$ & - & [19] \\
\hline \multirow[t]{7}{*}{$7 / 2$} & 34243.714 & $655(2)$ & & 3608.018 & $\mathrm{nl}, \mathrm{f}$ & 1 & $7 / 2$ & 6535.572 & 979 (1) & $25(30)$ & [19] \\
\hline & & & & 4286.343 & $\mathrm{nl}, \mathrm{e}$ & 1 & $9 / 2$ & 10920.365 & $632(2)$ & - & [19] \\
\hline & & & & 4713.198 & $\mathrm{nl}, \mathrm{f}$ & 1 & $7 / 2$ & 13032.634 & $893(3)$ & - & [19] \\
\hline & & & & 4734.423 & $\mathrm{nl}, \mathrm{f}$ & 1 & $5 / 2$ & 13127.722 & $156(1)$ & $0(10)$ & [19] \\
\hline & & & & 5763.922 & $\mathrm{e}$ & 20 & $9 / 2$ & 16899.229 & $216(2)$ & & This work \\
\hline & & & & 6502.982 & & 15 & $5 / 2$ & 18870.4 & $886(2)$ & & This work \\
\hline & & & & 7261.724 & $\mathrm{nl}$ & 4 & $9 / 2$ & 20476.674 & $550(1)$ & $50(50)$ & This work \\
\hline \multirow[t]{6}{*}{$17 / 2$} & 35453.400 & $595(1)$ & & 3888.734 & $\mathrm{f}$ & 6 & $15 / 2$ & 9745.376 & $540(2)$ & - & {$[4]$} \\
\hline & & & & 4011.474 & $\mathrm{nl}, \mathrm{f}$ & 1 & $17 / 2$ & 10531.951 & $546(3)$ & - & [19] \\
\hline & & & & 5052.260 & $\mathrm{nl}, \mathrm{f}$ & 8 & $17 / 2$ & 15665.796 & $906(1)$ & - & [19] \\
\hline & & & & 5629.604 & $\mathrm{nl}, \mathrm{f}$ & 1 & $17 / 2$ & 17695.093 & $1059(3)$ & - & [19] \\
\hline & & & & 5820.62 & $\mathrm{nl}, \mathrm{e}$ & 1 & $15 / 2$ & 18277.874 & $581(3)$ & - & [19] \\
\hline & & & & 5848.02 & $\mathrm{nl}, \mathrm{e}$ & 1 & $15 / 2$ & 18358.336 & $477(3)$ & - & [19] \\
\hline
\end{tabular}

C: Comment; nl: new line; e: excited; f: observed as fluorescence line. Lines, for which SNR 1 is given, do not show up in our FT spectrum but could be excited or were observed as fluorescence lines.

as transition between levels having $J_{\text {up }}=11 / 2$ and $J_{\mathrm{lo}}=$ 9/2 with $A_{\text {up }}=370 \mathrm{MHz}$ and $A_{\mathrm{lo}}=1365 \mathrm{MHz}$. The cg wavelength of the structure was $5826.37 \pm 0.01 \AA$. The known lower level $11730.668 \mathrm{~cm}^{-1}$, odd parity, had compatible values, and we calculated an energy of $28889.29 \mathrm{~cm}^{-1}$ for a hypothetical new even parity level. The strong fluorescence line could be explained as decay to the lower level $9579.820 \mathrm{~cm}^{-1}$, odd parity, $J=9 / 2$. This interpretation was confirmed thereafter by three further laser excitations $(\lambda=$ $5690.96,5693.32$ and $5831.69 \AA$ ). Figure 2 shows part of the FT spectrum around the line $5826.37 \AA$. The accuracy of the cg wavelengths is determined by our lambdameter to be $0.01 \AA$. This level explains one further line in the FT spectrum with respect to wavelength and hf pattern, $\lambda=6817.989 \AA$ (cg wavelength from FT spectrum).

When we tried to determine the energy of the level with higher precision, it turned out that we could not find suitable lines in our FT spectrum: none of the 4 excited transitions appears in the FT spectrum. The line $\lambda=6817.989 \AA$ (SNR
$=4$ in the FT spectrum) is a transition to a lower level whose energy is not accurately known, and the quite strong line $\lambda=5177.373 \AA$ (SNR $\approx 220)$ is blended by another strong line. Nevertheless, we tried to use the high wavelength accuracy of the FT spectrum $(0.001 \AA$ compared to $0.01 \AA$ of our lambdameter) for a determination of the excitation wavelength. As can be noticed in Figure 3(a), the interesting wavelength is in the neighborhood of a well-resolved line (cg wavelength $\lambda=5826.461 \AA$ ). We scanned the laser frequency over this line and detected a strong LIF signal at $\lambda_{\mathrm{fl}}=$ $5107 \AA$. After having passed the first 3 strong hyperfine components of this line, we switched the monochromator to the fluorescence line $\lambda_{\mathrm{fl}}=5177 \AA$, without interrupting the scan. Thus we obtained a recording in which the signals received on both fluorescence lines were contained next to one another (Figure 3(b)).

Using our fit program and the known hf constants of all 4 involved levels, it was possible to find the wave number 


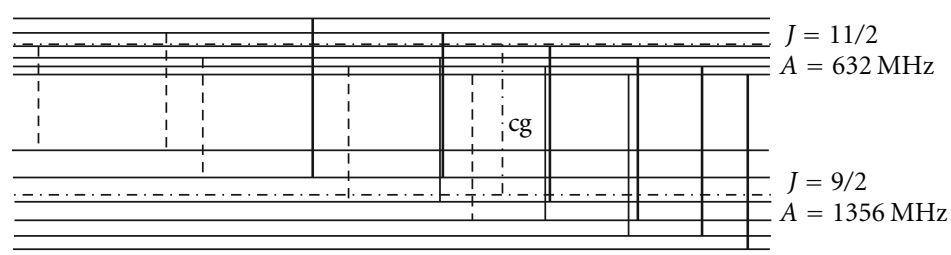

(d)

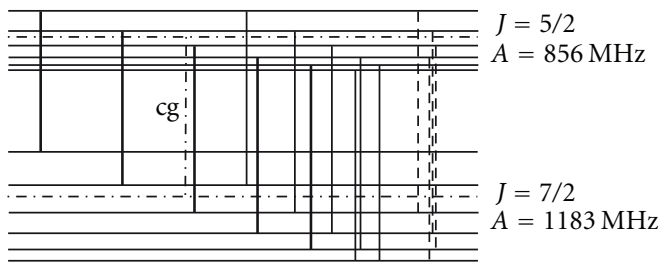

(c)
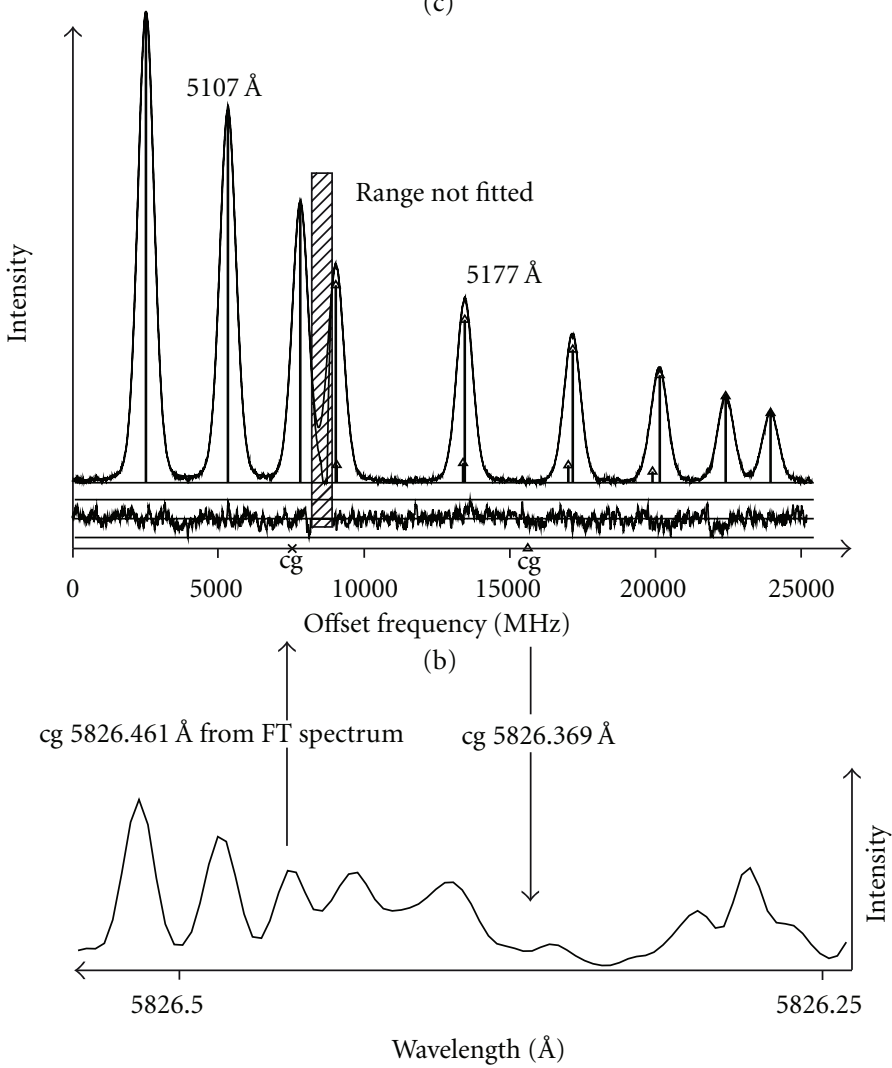

(a)

Figure 3: Finding of the cg wavelength of line $\lambda=5826.369 \AA$. (a) Part of the FT spectrum showing the well-resolved line $\lambda=5826.461 \AA$, but not the excited line $\lambda=5826.369 \AA$. (b) Laser spectroscopic scan over the wavelength region shown in (a). The left part (0-9000 MHz) was detected at fluorescence wavelength $\lambda_{\mathrm{fl}}=5107 \AA$, the right part at $\lambda_{\mathrm{fl}}=5177 \AA$. Each part was fitted using the hf structure constants of the corresponding combining level pairs (components outside the detected range were excluded). Lower trace: residuals between recording and fit, magnified by a factor 3.5. From the frequency difference between the centers of gravity, we could find the unknown excitation wavelength $\lambda=5826.369 \AA$. (c) Hyperfine level scheme of the line $\lambda=5826.461 \AA$. (d) Hyperfine level scheme of the line $\lambda=5826.369 \AA$.

difference of the cg's of both lines with high precision. By adding this value to the cg wave number of the line $\lambda=$ $5826.461 \AA$, we were able to find an accurate value for the cg wave number of the excitation to level $28889 \mathrm{~cm}^{-1}$. The corresponding wavelength is $\lambda=5826.369$ (1) $\AA$. With the new cg wave number, the energy of the new level was determined to be $28889.260(10) \mathrm{cm}^{-1}$. For confirmation, similar procedures were performed with two other excitations, and we found the wavelengths $\lambda=5831.692$ and $\lambda=5693.323 \AA$, which both confirmed the energy of this new level.

\section{Results}

In Table 1 we report data of low-lying even levels, discovered as lower levels of excited transitions. In columns 1 to 3 , values 
of $J$, the energy, and the A-factor are given. Columns 46 contain all lines known to us who can be classified with help of this level. The wavelength in column 4 is given with 3 digits after the decimal point if it is extracted from our FT spectrum. Two digits are given for excitation wavelengths measured with help of our lambdameter (accuracy $0.01 \AA$ ). For fluorescence lines having SNR $=1$, the wavelengths calculated from the level energies are given. All wavelengths are given in air. In column 5, a comment is entered: $\mathrm{nl}$ means that it is a new line, not contained in commonly used spectral tables (e.g., [18]). If $\mathrm{cl}$ is given, it is an already known line which could be classified with help of the new level. e indicates that the line was excited, $f$ that it was observed as a fluorescence line after excitation of the upper combining level. In column 6 the SNR of the line in our FT spectrum is given. If there is given $\mathrm{SNR}=1$, the line does not appear in the FT spectrum, but was observed as excited (comment e) or as fluorescence line (comment $\mathrm{f}$ ). In columns 7 to 11 , information on the combining upper level is entered. The citations in column 11 refer to the $A$ and $B$-values of columns 9 and 10. If a line of column 4 was excited, we additionally give in columns 12 to 16 information concerning the observed fluorescence line(s). $\mathrm{nl}$ and $\mathrm{cl}$ in column 13 have the same meaning as in column 5.

Table 2 contains data of low-lying odd levels, discovered as lower levels of excited transitions, and is built similar to Table 1.

In Table 3 all levels discovered as upper levels are listed, divided in two groups: even parity and odd parity. Within each group, the levels are sorted with respect to $J$ (column 1) and for each $J$ with increasing energy (column 2). Column 3 contains the A-factor and column 4 the B-factor if we were able to determine it reliably. In column 5, excitation wavelengths are given (not more than 2) and in column 6 fluorescence wavelengths (not more than 2).

Table 4 contains detailed information concerned with the new even levels. The table is built analogously to Table 1 (but here the columns 12 to 16 of Table 1 are not needed).

Table 5 contains detailed information concerned with the new odd levels and is built similarly to Table 4 .

\section{References}

[1] R. M. Macfarlane, D. P. Burum, and R. M. Shelby, "New determination of the nuclear magnetic moment of ${ }^{141} \mathrm{Pr}$," Physical Review Letters, vol. 49, no. 9, pp. 636-639, 1982.

[2] K. D. Böklen, T. Bossert, W. Foerster, H. H. Fuchs, and G. Nachtsheim, "Hyperfine structure measurements in the ${ }^{4} \mathrm{I}_{9 / 2}$ ground state of ${ }^{141} \mathrm{Pr}$," Zeitschrift für Physik A, vol. 274, no. 3, pp. 195-201, 1975.

[3] W. C. Martin, R. Zalubas, and L. Hagan, Atomic Energy Levels - The Rare Earth Elements National Bureau of Standards, vol. 60, NSRDS-NBS, Washington, DC, USA, 1978.

[4] A. Ginibre, "Fine and hyperfine structures in the configurations $4 \mathrm{f}^{2} 5 \mathrm{~d} 6 \mathrm{~s}^{2}$ and $4 \mathrm{f}^{2} 5 \mathrm{~d}^{2} 6 \mathrm{~s}$ of neutral praseodymium," Physica Scripta, vol. 23, no. 3, p. 260, 1981.

[5] A. Ginibre, "Classification et étude paramétrique des spectres complexes á l'aide de l'interprétation des structures hyperfines: spectres I et II du praséodyme," [Ph.D. thesis], Université de Paris-Sud, Centre d'Orsay Paris, 1988.
[6] A. Ginibre, "Fine and hyperfine structures of singly ionized praseodymium: I. energy levels, hyperfine structures and Zeeman effect, classified lines," Physica Scripta, vol. 39, no. 6, p. $694,1989$.

[7] W. J. Childs and L. S. Goodman, "Double resonance, fluorescence spectroscopy, and hyperfine structure in Pr I," Physical Review A, vol. 24, no. 3, pp. 1342-1349, 1981.

[8] T. Kuwamoto, I. Endo, A. Fukumi et al., "Systematic study of fine and hyperfine structures in Pr I by doppler-free atomicbeam laser spectroscopy," Journal of the Physical Society of Japan, vol. 65, no. 10, pp. 3180-3185, 1996.

[9] A. Krzykowski, B. Furmann, D. Stefánska, A. Jarosz, and A. Kajoch, "Hyperfine structures in the configuration $4 \mathrm{f} 35 \mathrm{~d} 6 \mathrm{~s}$ of the praseodymium atom," Optics Communications, vol. 140, no. 4-6, pp. 216-219, 1997.

[10] B. Furmann, A. Krzykowski, D. Stefańska, and J. Dembczyński, "New levels and hyperfine structure evaluation in neutral praseodymium," Physica Scripta, vol. 74, no. 6, pp. 658-669, 2006.

[11] J. Ruczkowski, E. Stachowska, M. Elantkowska, G. H. Guthöhrlein, and J. Dembczyński, "Interpretation of the hyperfine structure of the even configuration system of Pr I," Physica Scripta, vol. 68, no. 2, pp. 133-140, 2003.

[12] B. Gamper, Z. Uddin, M. Jahangir et al., "Investigation of the hyperfine structure of Pr I and Pr II lines based on highly resolved Fourier transform spectra," Journal of Physics B, vol. 44, no. 4, Article ID 045003, 2011.

[13] K. Shamim, I. Siddiqui, and L. Windholz, "Experimental investigation of the hyperfine spectra of Pr I-clines: discovery of new fine structure levels with low angular momentum," The European Physical Journal D, vol. 64, no. 2-3, pp. 209-220, 2011.

[14] T. I. Syed, I. Siddiqui, K. Shamim, Z. Uddin, G. H. Guthöhrlein, and L. Windholz, "New even and odd parity levels of neutral praseodymium," Physica Scripta, vol. 84, Article ID 065303, 2011.

[15] U. Zaheer, S. Imran, S. Khan, B. Gamper, E. Abdul-Hafidh, and L. Windholz, "New levels of the Pr atom with almost similar energies," Journal of Physical Science and Application, vol. 2, no. 4, pp. 88-94, 2012.

[16] T. A. Ryabchikova, I. S. Savanov, V. P. Malanushenko, and D. O. Kudryavtsev, "A study of rare earth elements in the atmospheres of chemically peculiar stars. Pr III and Nd III lines ," Astronomy Reports, vol. 45, no. 5, pp. 382-388, 2001.

[17] S. Ivarsson, U. Litzen, and G. M. Wahlgren, "Accurate wavelengths, oscillator strengths and hyperfine structure in selected praseodymium lines of astrophysical interest," Physica Scripta, vol. 64, no. 5, p. 455, 2001.

[18] G. R. Harrison, Wavelength Tables, Massachusetts Institute of Technology (M.I.T. Press), 1969.

[19] Helmut-Schmidt-Universität, Universität der Bundeswehr Hamburg, and Laboratorium für Experimentalphysik, unpublished material taken from several diploma theses (supervisor G. H. Guthöhrlein). 

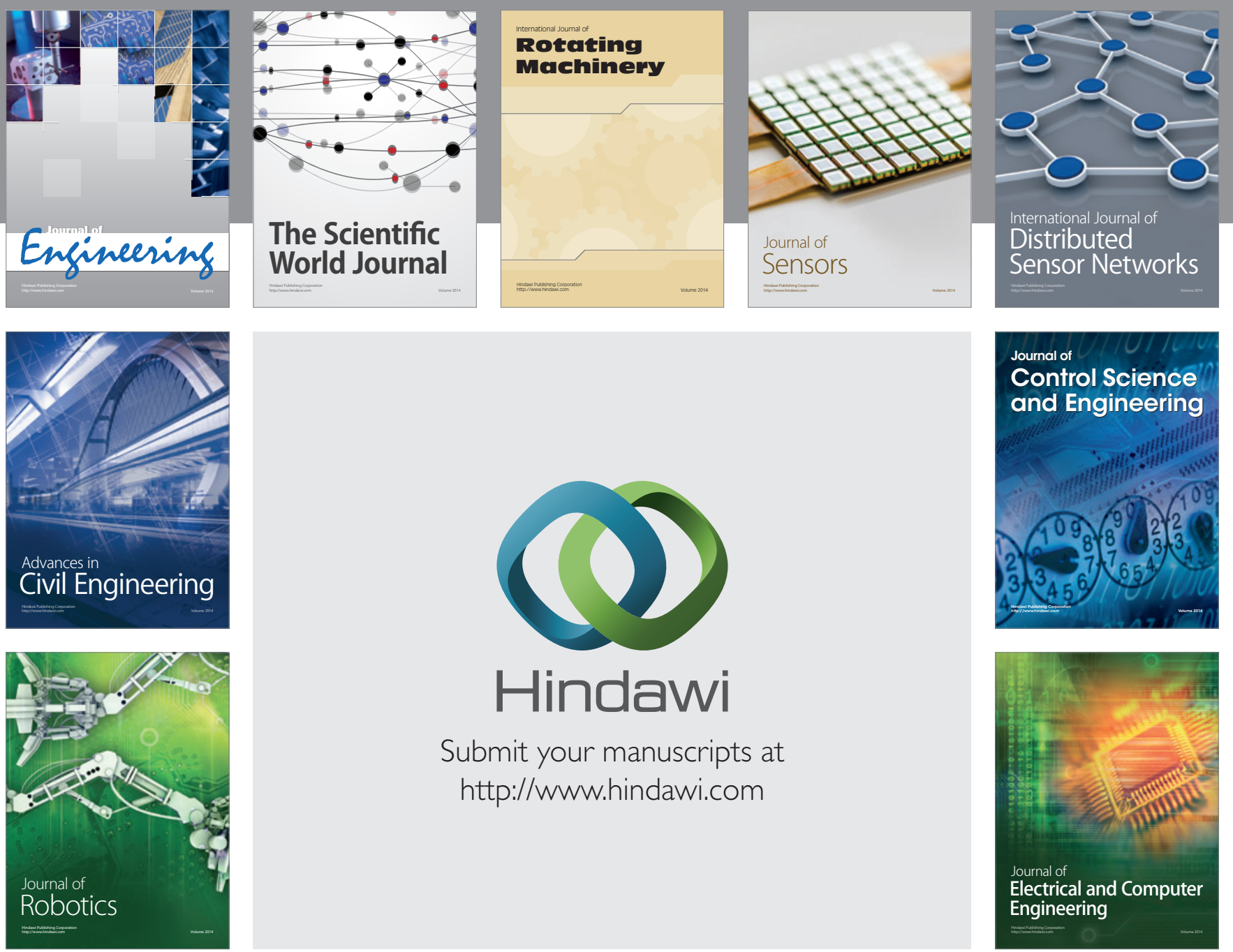

Submit your manuscripts at

http://www.hindawi.com
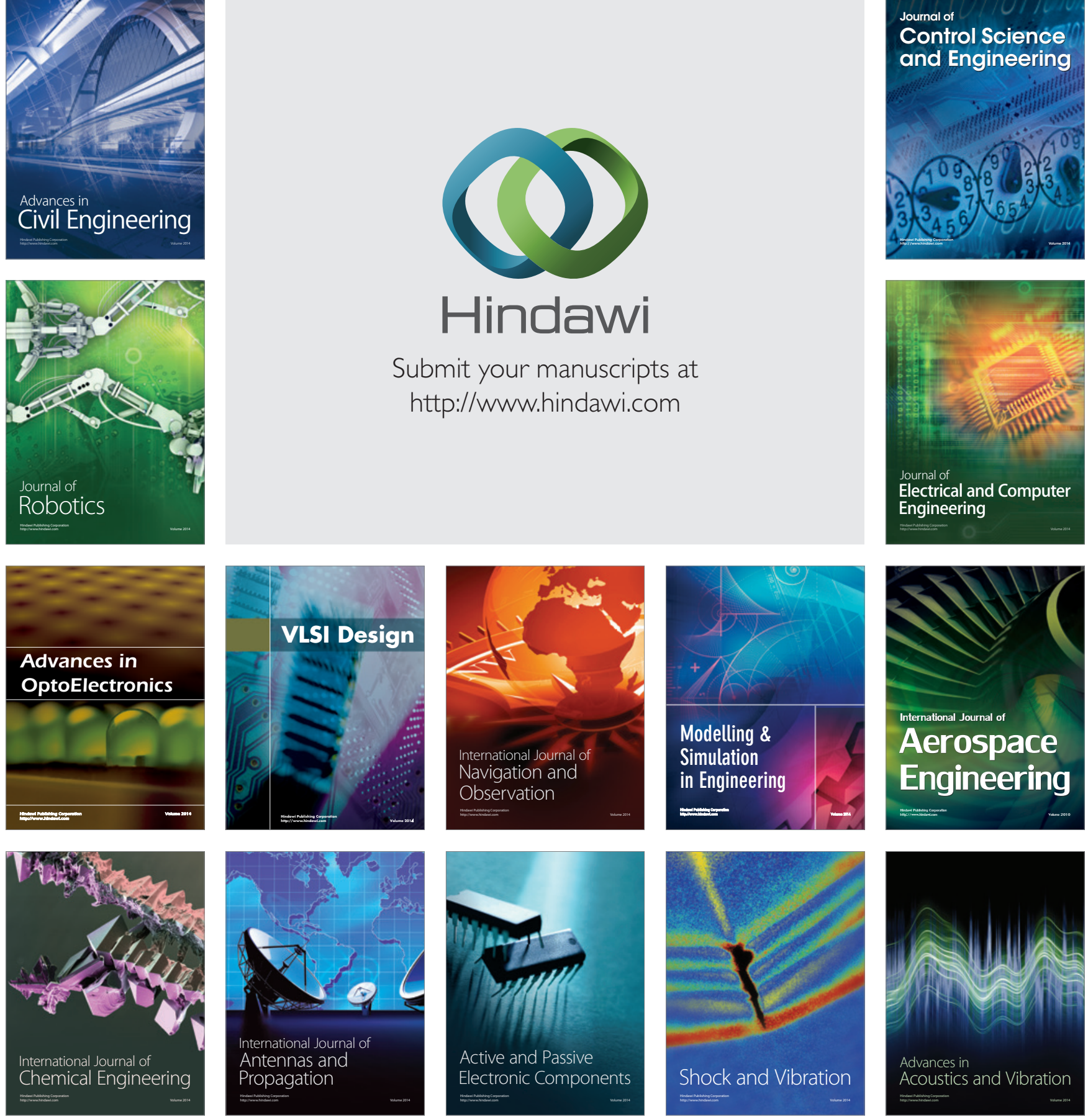